\title{
A Wind Tunnel Study of Atmospheric Boundary Layer Effects on Roof Mounted Solar Panel Arrays
}

by

\author{
Curtis Arends
}

A Masters Thesis submitted to the Faculty of Graduate Studies and Research

in partial fulfilment of the requirements for the degree of

Master of Applied Science in Aerospace Engineering

Department of Mechanical and Aerospace Engineering Carleton University

Ottawa, Ontario, Canada

September 2011

(C) Curtis Arends, 2011 
Library and Archives Canada

Published Heritage Branch

395 Wellington Street Ottawa ON K1A ON4 Canada
Bibliothèque et

Archives Canada

Direction du

Patrimoine de l'édition

395 , rue Wellington

Ottawa ON K1A ON4

Canada
Your file Votre référence
ISBN: $978-0-494-83063-5$
Our file Notre référence
ISBN: $978-0-494-83063-5$
NOTICE:

The author has granted a nonexclusive license allowing Library and Archives Canada to reproduce, publish, archive, preserve, conserve, communicate to the public by telecommunication or on the Internet, loan, distribute and sell theses worldwide, for commercial or noncommercial purposes, in microform, paper, electronic and/or any other formats.

The author retains copyright ownership and moral rights in this thesis. Neither the thesis nor substantial extracts from it may be printed or otherwise reproduced without the author's permission.
AVIS:

L'auteur a accordé une licence non exclusive permettant à la Bibliothèque et Archives Canada de reproduire, publier, archiver, sauvegarder, conserver, transmettre au public par télécommunication ou par l'Internet, prêter, distribuer et vendre des thèses partout dans le monde, à des fins commerciales ou autres, sur support microforme, papier, électronique et/ou autres formats.

L'auteur conserve la propriété du droit d'auteur et des droits moraux qui protège cette thèse. $\mathrm{Ni}$ la thèse ni des extraits substantiels de celle-ci ne doivent être imprimés ou autrement reproduits sans son autorisation.
In compliance with the Canadian Privacy Act some supporting forms may have been removed from this thesis.

While these forms may be included in the document page count, their removal does not represent any loss of content from the thesis.
Conformément à la loi canadienne sur la protection de la vie privée, quelques formulaires secondaires ont été enlevés de cette thèse.

Bien que ces formulaires aient inclus dans la pagination, il n'y aura aucun contenu manquant.

\section{Canadä}


The undersigned recommend to the Faculty of Graduate Studies and Research acceptance of this Masters Thesis

\title{
A Wind Tunnel Study of Atmospheric Boundary Layer Effects on Roof Mounted Solar Panel Arrays
}

\author{
Submitted by Curtis Arends \\ in partial fulfilment of the requirements for the degree of \\ Master of Applied Science \\ in Aerospace Engineering
}

F. Nitzsche, Supervisor

E. Matida, Supervisor

M. Yaris, Department Chair

Carleton University

2011 


\section{Abstract}

With the rising cost of conventional fossil fuels, alternative energies such as solar power are becoming more attractive. With government incentives, private companies are being encouraged to transfer their power demand from the electrical grid to on site power generation from solar sources. Incentives are also in place to enable developers to push solar panel array products to market for widespread and large scale installations. Installing solar arrays on roof top structures requires confirmation from the National Building Code that the roof top installation is safe and legal. However, due to the unique geometry and dynamic nature of solar panels, specifically those that track the movement of the sun, the National Building Code requires experimental validation of the installations' safety. This thesis examines the current nature of the solar industry, and the means in which the national building code of Canada supports it. A review of fluid fundamentals, the atmospheric boundary layer, and computational principles will prepare the reader for understanding the project's experiments. The research will examine the operating conditions that such a solar panel array on the roof of a large retail building may experience, and comment on the limitations that exist for practical full scale models while exposed to wind, known as the earth's atmospheric boundary layer. Using a load cell, measurements of forces and bending moments on scaled solar panel models were taken inside a low speed atmospheric boundary layer wind tunnel with the panel mounted on the roof of a scaled retail building. These measurements were used to validate two dimensional 
large eddy simulations on a computer. Differences present in the results are mainly due to the nature of the solar panel's and retail building's three dimensional effects. Vortex shedding was also observed and commented upon. It was confirmed that force and moment coefficients obtained during wind tunnel testing are insensitive to Reynolds numbers, and may thus be used for full scale model calculations. A variety of trends were noted from the wind tunnel measurements regarding the effect of numerous panel configurations on the roof top. A conclusion is finally made regarding the feasibility of the research and its application to future studies. 
Dedicated to my family and close friends, for their never ending prayer, support, and love. 


\section{Acknowledgments}

My first acknowledgment belongs to those who made this project possible, namely my co-supervisors Doctor Fred Nitzsche, Doctor Edgar Matida, and Doctor Daniel Feszty from Carleton University's Department of Mechanical and Aerospace Engineering. This project would not have been possible without the knowledge and financial support I received from them.

PhD candidate Sean McTavish from Carleton University deserves a very special thanks for his work in setting up the atmospheric boundary layer wind tunnel and showing me how to use it and how it works. His persistence to create a functional and reliable experimental atmosphere was very encouraging, and I will never forget those long hours together with you underneath the wind tunnel floor moving the pitot tube inch by inch into the air-stream to measure the boundary layer thickness. My poor back will never forget it either.

The acrodynamics lab team at the National Research Council's Institute of Aerospace Research cannot go without mention. Doctor Hongyi Xu, Doctor Alanna Wall, and Doctor Brian McAuliffe each showed me the life of a researcher, and the details that are taken at every step in a research project. Thank you for giving me the opportunity to work in such a fine establishment.

Behind the scenes I cannot forget to mention the department staff Christie Egbert, Nancy Powell, and the late Marlene Groves. Their assistance from the beginning made everything go so much smooth, from initial questions about the process of getting into 
grad school, to sending faxes, to making purchases, and for getting access to rooms. You made it possible, and I thank you.

It is hard to forget the friendly faces of the machine shop crew in the Mechanical and Aerospace Department. Alex Proctor, Kevin Sangster, and Steve Truttman somehow put up with my persistent presence in the machine shop, while constantly teaching me new machine skills and assembly ideas. Without them, I would never have achieved the wind tunnel apparatus that I ended up with. Thanks guys.

Rob Williams, a summer intern student, made a breakthrough with the project early on with the discovery of the force plate load cell. His find, although quite expensive, greatly benefited the project. His insight into how the experiment could be setup in the wind tunnel was invaluable, and the brainstorming sessions with him were truly appreciated.

The years only went by so smoothly with the continual support of my family, Paul, Catherine, Jessica, and Kyle Arends. God used them in my life in ways that have truly changed me, and my entire perspective life. This leads me to my wonderful girlfriend, Leah Mooney. I may have a degree in Aerospace Engineering, but she will always be smarter than me. Thank you Leah for always being excited whenever I made just a small bit of progress, and for helping me back on my feet when things did not go very well.

Finally, nothing compares to the grace and mercy I have received from God. Regardless of the many times I have fallen, the times I have turned my back on Him for my own selfish gain, I am confident and so thankful that I am forgiven through His sacrifice for me. I deserve eternal separation from God, but Christ has taken my punishment upon Himself on my behalf. With a restored relationship with God, and Him working through me to make me a better man to serve others, I am forever thankful. This gift, so true, is free to you also, by faith in Him alone. 


\section{Table of Contents}

$\begin{array}{ll}\text { Abstract } & \text { iii }\end{array}$

List of Tables $\quad$ xii

List of Figures $\quad$ xiii

Nomenclature $\quad$ xvi

1 Introduction $\quad 1$

1.1 Solar Industry . . . . . . . . . . . . . . . . . . . 1

1.2 Collaboration between Carleton University, the Ontario Government, and the National Research Council . . . . . . . . . . . . . 2

1.3 Dual Axis Solar Tracking . . . . . . . . . . . . . . . . . 2

1.4 The Need for Experimental Testing . . . . . . . . . . . . . . 3

1.5 Research Goals ... . . . . . . . . . . . . . . . . 4

2 Roof Structures in the National Building Code of Canada 6

2.1 Specified Loads . . . . . . . . . . . . . . . . 6

2.2 Wind Loads . . . . . . . . . . . . . . . . . . . . . 7

2.2.1 Calculating Wind Effects . . . . . . . . . . . . . . 11

2.3 Structural Equivalents . . . . . . . . . . . . . . 12 


\section{Fluid Dynamics Background}

3.1 Fluid Forces and Moments on Flat Plates . . . . . . . . . . . 13

3.2 Forces on Panels Mounted in Turbulence . . . . . . . . . . . . . 14

3.3 Understanding Vortex Shedding . . . . . . . . . . . . . . . 16

4 The Atmospheric Boundary Layer $\quad 18$

4.1 Origin of the Wind $\ldots \ldots \ldots \ldots \ldots \ldots$

4.1.1 Isotropic Turbulence and Parallel Shear Motion . . . . . . 19

4.1.2 Mean Flow in Atmospheric Boundary Layer . . . . . . . . 19

4.2 Determination of Design Wind Velocities . . . . . . . . . . . 21

4.3 Response of a Structure to Wind . . . . . . . . . . . . . . . 23

4.3.1 Static Analysis of a Rooftop Structure . . . . . . . . . 24

4.4 Designing an Atmospheric Boundary Layer . . . . . . . . . . . . . 25

5 Wind Tunnel Setup $\quad 28$

5.1 Wind Tunnel and Test Configurations . . . . . . . . . . . 28

5.1 .1 The Wind Tunnel . . . . . . . . . . . . . . . . . 28

5.1 .2 The Test Section $\ldots \ldots \ldots \ldots \ldots \ldots$

5.1 .3 Test Configurations . . . . . . . . . . . . . . 33

5.2 Data Acquisition . . . . . . . . . . . . . . . . . 39

5.2 .1 Multi-axis force platform . . . . . . . . . . . . . 39

5.2 .2 Acquisition Software . . . . . . . . . . . . . 42

5.2 .3 Platform Calibration . . . . . . . . . . . . . . . . 45

5.3 Uncertainty Analysis . . . . . . . . . . . . . . . . . . . . 54

5.4 Sources of Error . . . . . . . . . . . . . . . . 56

5.4 .1 Design Considerations $\ldots \ldots \ldots \ldots \ldots$

5.4 .2 Human Error . . . . . . . . . . . . . . . . . 57 
6.1 Theoretical Vortex Frequency . . . . . . . . . . . . . . 61

6.2 Effect of Varying Wind Velocity . . . . . . . . . . . . . . 61

6.2 .1 Significance of Wind Velocity $\ldots \ldots \ldots . \ldots 63$

6.2 .2 Reynolds Number Insensitivity _ . . . . . . . . . . . 63

6.3 Effect of Varying Panel Elevation Angle . . . . . . . . . . . . . 65

6.3.1 Significance of Panel Elevation Angle . . . . . . . . . . 66

6.4 Effect of Varying Panel Azimuth Angle . . . . . . . . . . . . . 67

6.4.1 Significance of Panel Azimuth Angle . . . . . . . . . . . . 68

6.5 Effect of Varying Wind Direction . . . . . . . . . . . . . 69

6.5.1 Significance of Wind Direction . . . . . . . . . . . . 70

6.6 Effect of Varying Panel Rooftop Locations . . . . . . . . . . . . 71

6.6.1 Significance of Panel Rooftop Location . . . . . . . . . . . 72

6.7 Effect of Nearby Panels . . . . . . . . . . . . . . . . . . . . . 73

6.7.1 Significance of Nearby Panels . . . . . . . . . . . . . 74

6.8 Effect of Panel Submersion in a Field of Panels . . . . . . . . . . 74

6.8.1 Significance of Panel Fields $\ldots \ldots \ldots \ldots$

$\begin{array}{lll}7 & \text { Computational Simulation Setup } & 77\end{array}$

7.1 Introducing Randomized Turbulence . . . . . . . . . . . 77

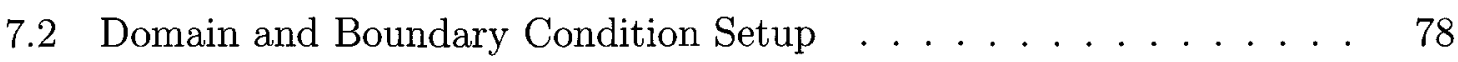

7.2 .1 Boundary Conditions . . . . . . . . . . . . . 78

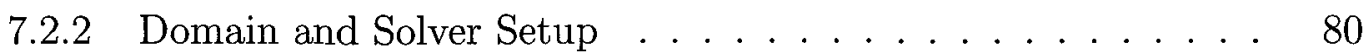

7.3 Domain Sizing . . . . . . . . . . . . . . . . . . . 80

7.4 Grid Density . . . . . . . . . . . . . . . . . 84

8 Computational Results and Discussions $\quad 89$

8.1 2D LES Forces and Torque . . . . . . . . . . . . . . . . . . . . 89 
8.2 LES Frequency Analyses . . . . . . . . . . . . . . . . . . . . 93

8.2.1 Wind Tunnel Frequency Analyses . . . . . . . . . . . . 94

8.2.2 Comparison of LES to Wind Tunnel Data . . . . . . . . 95

9 Conclusions $\quad 98$

9.1 Future Testing and Design Considerations . . . . . . . . . . 100

9.1.1 Significance of Snow Accumulation . . . . . . . . . . . 100

9.1.2 Significance of Model Variation . . . . . . . . . . 100

9.1.3 Panel Characteristics without Building . . . . . . . . . . 101

9.1.4 Improve Model Assumptions . . . . . . . . . . . . . . . . 101

9.1.5 Panel Modification Suggestions . . . . . . . . . . 102

9.1 .6 FEM stress analysis . . . . . . . . . . . . . 102

9.1.7 Aeroelastic Effects . . . . . . . . . . . . . . . . 103

$\begin{array}{ll}\text { List of References } & 104\end{array}$

$\begin{array}{ll}\text { Appendix A } & 106\end{array}$

$\begin{array}{ll}\text { Appendix B Wind Tunnel Results Matrix } & 110\end{array}$

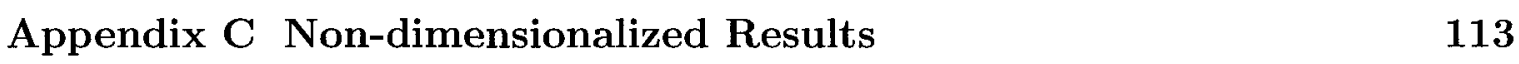

Appendix D Computational Fluid Dynamics Background $\quad 116$

D.1 Modelling Turbulence using RANS and LES . . . . . . . . . 116

D.2 Finite Volume Method . . . . . . . . . . . . . 117

D.2.1 Central Differencing Finite Volume Advection Scheme . . . . 119

D.3 LES Flow Around Bluff Bodies . . . . . . . . . . . . . . 120 


\section{List of Tables}

2.1 Importance categories for buildings $\ldots \ldots \ldots \ldots 7$

2.2 Importance factor for wind load $\ldots \ldots \ldots \ldots$

4.1 Coefficients for boundary layer shape $\ldots \ldots \ldots \ldots 23$

8.1 RANS forces and torque $\ldots \ldots \ldots \ldots \ldots$ 


\section{List of Figures}

3.1 Strouhal number of flat plate at incidence $\ldots \ldots \ldots \ldots$

4.1 Profiles over differing terrain roughness . . . . . . . . . . . 20

4.2 Distance needed to establish new wind profile after roughness change 21

4.3 Increase of velocity with height over different terrain . . . . . . . . 22

4.4 Wind tunnel spires and roughness elements . . . . . . . . . . . . 25

5.1 Upwind view from test section . . . . . . . . . . . . . . . . . . 29

5.2 Downwind view of test section . . . . . . . . . . . . . . . . . . 29

5.3 Wind Tunnel Diagram . . . . . . . . . . . . . . . . . . 30

5.4 Test section initial panel setup . . . . . . . . . . . . . . . 31

5.5 Load cell setup underneath building . . . . . . . . . . . . . . . . 32

5.6 Side view of experiment setup . . . . . . . . . . . . 33

5.7 Rooftop layout . . . . . . . . . . . . . . . . . . . 34

5.8 Changing wind direction $\ldots \ldots \ldots \ldots \ldots \ldots$

5.9 Panel angles . . . . . . . . . . . . . . . . . . . 36

5.10 Nearby dummy panel at position $\mathrm{N} \ldots \ldots \ldots$

5.11 Panel submersion in small panel field . . . . . . . . . . . . . 37

5.12 Panel submersion in large panel field . . . . . . . . . . . . . . . 38

5.13 Panel submersion in sparse panel field . . . . . . . . . . . . . . . . 39

5.14 HE6X6 low load miniature force platform . . . . . . . . . . . . 40

5.15 Orientation of force plate to wind tunnel flow $\ldots \ldots \ldots$ 
5.16 Hall-Effect sensor locations . . . . . . . . . . . . . . . . . . . 41

5.17 Force plate hardware layout . . . . . . . . . . . . . . . 42

5.18 Platform setup for $F_{z}$ calibration $\ldots \ldots \ldots \ldots$

5.19 Measured vs Actual Z-force . . . . . . . . . . . . . . 46

5.20 Platform setup for $F_{x}$ calibration $\ldots \ldots \ldots \ldots$

5.21 Measured vs Actual X-force . . . . . . . . . . . . . . . 47

5.22 Platform setup for $F_{y}$ calibration $\ldots \ldots \ldots \ldots \ldots$

5.23 Measured vs Actual Y-force . . . . . . . . . . . . . . . . 49

5.24 Force calibration . . . . . . . . . . . . . . . . . 49

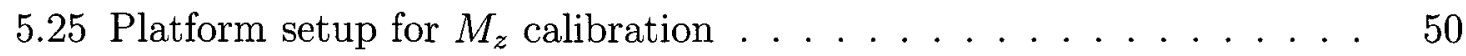

5.26 Measured vs Actual Z-moment . . . . . . . . . . . . . . . 51

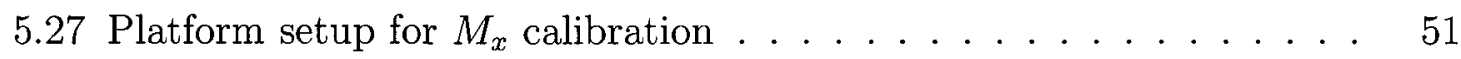

5.28 Measured vs Actual X-moment f . . . . . . . . . . . . . 52

5.29 Platform setup for $M_{y}$ calibration $\ldots \ldots \ldots \ldots \ldots$

5.30 Measured vs Actual Y-moment . . . . . . . . . . . . . 53

5.31 Moment calibration . . . . . . . . . . . . . . . . . 54

5.32 Estimated uncertainty of forces . . . . . . . . . . . 55

5.33 Estimated uncertainty of moments . . . . . . . . . . . 56

6.1 Example of schematic for velocity test case . . . . . . . . . . . 62

6.2 Wind velocity reaction forces . . . . . . . . . . . . . . 62

6.3 Wind velocity reaction moments . . . . . . . . . . . . 63

6.4 Panel elevation angle reaction forces $\ldots \ldots \ldots \ldots$

6.5 Panel elevation angle reaction moments . . . . . . . . . . . . 66

6.6 Panel azimuth angle reaction forces . . . . . . . . . . 67

6.7 Panel azimuth angle reaction moments $\ldots \ldots \ldots \ldots$

6.8 Wind direction reaction forces . . . . . . . . . . . . . . . 69

6.9 Wind direction reaction moments . . . . . . . . . . 70 
6.10 Panel position reaction forces . . . . . . . . . . . . . 71

6.11 Panel position reaction moments. . . . . . . . . . . . 72

6.12 Adjacent panel position reaction forces . . . . . . . . . . 73

6.13 Adjacent panel position reaction moments . . . . . . . . . . . . 74

6.14 Panel field reaction forces . . . . . . . . . . . . . . . 75

6.15 Panel field reaction moments . . . . . . . . . . . . . . . . 75

7.1 Wind tunnel simulated atmospheric boundary layer . . . . . . . . . . 79

7.2 Final domain size . . . . . . . . . . . . . . . . 81

7.3 Downstream domain sizing . . . . . . . . . . . . . . . . 82

7.4 Domain ceiling sizing . . . . . . . . . . . . . . . . . 83

7.5 Upstream domain sizing .................. 84

7.6 Initial mesh with converged domain . . . . . . . . . . . 85

7.7 RANS grid convergence . . . . . . . . . . . . . 86

7.8 RANS converged mesh . . . . . . . . . . . . . . . . 87

7.9 LES grid convergence . . . . . . . . . . . . . . . . . . 87

8.1 Steady state RANS . . . . . . . . . . . . . . . . . 90

8.2 Example of vortex shedding . . . . . . . . . . . . . . 91

8.3 Panel forces over time . . . . . . . . . . . . . . . . . . . . 92

8.4 Panel torque over time . . . . . . . . . . . . . . . 93

8.5 LES fluctuations . . . . . . . . . . . . . . . . . 94

8.6 LES frequency spectrum . . . . . . . . . . . . . . . . 94

8.7 Wind tunnel fluctuations . . . . . . . . . . . . . . 95

8.8 Wind tunnel frequency spectrum . . . . . . . . . . . . . 95 


\section{Nomenclature}

Table N1 Abbreviations

\begin{tabular}{ll}
\hline \hline LES & Large Eddy Simulation \\
TDMA & Thomas' tridiagonal matrix algorithm \\
DNS & Direction numerical simulation \\
RANS & Reynolds Averaged Navier-Stokes \\
CFD & Computational Fluid Dynamics
\end{tabular}

\begin{tabular}{lll}
\hline Table N2 & Variable Notation & Units \\
\hline$\alpha$ & Angle of attack & $o$ \\
$L$ & Aerodynamic lift & $\mathrm{N}$ \\
$\mu$ & Fluid viscosity & $\mathrm{kg} /(\mathrm{sm})$ \\
$\phi$ & Azimuth angle & $o$ \\
$\rho$ & Fluid density & $\mathrm{kg} / \mathrm{m}^{3}$ \\
$R e$ & Reynolds number & $\mathrm{unitless}$ \\
$V$ & Scalar Velocity & $\mathrm{m} / \mathrm{s}$
\end{tabular}




\begin{tabular}{|c|c|c|}
\hline$\omega$ & Vortex frequency & $\mathrm{rad} / \mathrm{s}$ \\
\hline$W$ & Wind load & Newtons \\
\hline$p$ & Specified external pressure & $\mathrm{Pa}$ \\
\hline$I_{w}$ & importance factor for wind load & unitless \\
\hline$q$ & Reference velocity pressure & $\mathrm{Pa}$ \\
\hline$C_{e}$ & Exposure factor & unitless \\
\hline$C_{g}$ & Gust effect factor & unitless \\
\hline$C_{p}$ & External pressure coefficient & unitless \\
\hline$V_{H c}$ & Critical mean wind speed & $\mathrm{m} / \mathrm{s}$ \\
\hline$S$ & Strouhal number & unitless \\
\hline$f_{n}$ & Frequency & $\mathrm{Hz}$ \\
\hline$c$ & Panel chord length & $\mathrm{m}$ \\
\hline$V_{e f f}$ & Effective mean velocity in shear flow & $\mathrm{m} / \mathrm{s}$ \\
\hline$N$ & Normal force on panel in shear flow & $\mathrm{N}$ \\
\hline$S$ & Panel surface area & $\mathrm{m}^{2}$ \\
\hline$F x$ & Panel force in $\mathrm{X}$ direction & $\mathrm{N}$ \\
\hline$F y$ & Panel force in $Y$ direction & $\mathrm{N}$ \\
\hline$F z$ & Panel force in $\mathrm{Z}$ direction & $\mathrm{N}$ \\
\hline$M x$ & Panel torque on $\mathrm{x}$-axis & $\mathrm{N} / \mathrm{m}$ \\
\hline
\end{tabular}




\begin{tabular}{lll}
$M y$ & Panel torque on y-axis & $\mathrm{N} / \mathrm{m}$ \\
$M z$ & Panel torque on z-axis & $\mathrm{N} / \mathrm{m}$ \\
$k$ & Correction factors for mean forces & unitless \\
$\alpha$ & Terrain roughness coefficient & unitless \\
$V_{Z}$ & Wind velocity at height Z & $\mathrm{m} / \mathrm{s}$ \\
$V_{G}$ & Wind velocity at gradient height $\mathrm{G}$ & $\mathrm{m} / \mathrm{s}$ \\
$w$ & Earth's rotational speed & $\mathrm{rad} / \mathrm{s}$ \\
$d p / d N$ & Pressure gradient & $\mathrm{Pa} / \mathrm{m}$ \\
$\sigma$ & Boundary layer thickness & $\mathrm{m}$ \\
$D$ & Spacing of roughness elements & $\mathrm{m}$ \\
$k$ & Roughness height & $\mathrm{m}$ \\
$h$ & Spire height & $\mathrm{m}$ \\
\hline & Spire base length & $\mathrm{m}$ \\
\hline
\end{tabular}




\section{Chapter 1}

\section{Introduction}

\subsection{Solar Industry}

The province of Ontario has recognized the need for alternative energy sources to fossil fuels, and has set up programs to help get potentially less expensive, cleaner power into the grid. The Canadian government is offering incentives for companies to produce energy saving projects. These projects are not to be aimed at research initiatives, but for production of practical applications and large scale installations. The larger the project, the larger the payments will be from the ecoEnergy [1] program. The province is also awarding businesses funding to convert their traditional heating systems to those powered by solar. This influx of government incentives is spurring production of solar panel products. Additionally, the capability for an organization to sell electricity back to the public grid is a further incentive for this industry to grow. The additional benefit of producing or operating solar products is the green image that fosters a sense of positive awareness of the environment and the role that must be played by industries and individuals alike. Solar energy is a candidate that may be able to fulfill this desire for alternative energy, due to the energy from the sun being predictable and repeatable on a daily basis. Finally, many parts of the world have poor transmission of power throughout and in between communities. On 
site generation of this power through solar energy would help reach this need. Being able to offer a solar panel system that is fully developed and certified is the first step towards achieving this goal.

\subsection{Collaboration between Carleton University, the Ontario Government, and the National Research Council}

Under a grant from the Ontario Center of Excellence, Carleton University was partnered with an industry company to assist with experimental and computation verification of a solar panel array that could legal for installation under the National Building Code of Canada. This would provide a low cost method of performing broad, base level testing to determine critical design cases that must be examined. Under the guidelines by their supervisor, graduate students will be performing aerodynamic, computational, and aeroelastic testing on the solar array to determine important operating regimes. Furthermore, by collaborating with the Nation Research Council, fundamental computation techniques will be attained to assist and create a more accurately simulated flow environment.

\subsection{Dual Axis Solar Tracking}

Conventional solar panels are rigidly connected to the surface of a rooftop or support structure. This design, although relatively inexpensive to produce, is inefficient for collecting optimal energy from the sun, since the sun is continuously moving across the sky as the earth rotates, while the fixed panel is not directly exposed. A more efficient design would rotate the panel along the sun's azimuth. This would involve 
keeping the solar panel at a specific angle towards the sky, and rotating the panel along its vertical axis as the sun moves. This however still does not allow for the entire path of the sun to be tracked. For the panel to constantly face the sun directly, it must also track the sun's elevation angle. This would involve rotating the panel along its horizontal axis, and is approximately $30 \%$ more efficient at collecting energy than a fixed panel of the same surface area. Using auto-controlled motors run by GPS software would allow the solar panel array to remain fully automated and able to track the sun on its own every day. With these advantages there are also disadvantages that a customer would need to evaluate, such as the high upfront cost, and the density of panels able to be placed on a roof. The power density for a given roof area will be limited due to angled panel structures blocking sunlight to panels in their shadow. The complexity of hardware such as motors and movable structures is the main source of upfront costs, and would need to be weighed in conjunction with long term benefits on a case by case basis. However, since this method could likely be the type of solar panel used in industry, the dual axis solar tracker was put forward as the model to analyze for this project. Such an array, placed on the roof top of large retail stores, could reduce a significant amount of the building's usage of the electrical grid, whether placed individually on rooftops, or together in clusters of multiple panels. The design of such a system needs to be tested to ensure that it can withstand the exposure environment. It is a fine balance however to not remove the panel completely from wind forces, since the wind is useful for cooling the solar panels and allowing them to maintain their efficiency.

\subsection{The Need for Experimental Testing}

For a company to invest in a solar panel system, the equipment must be legal for installation and thus safe for its employees or customers. Although the National 
Building Code of Canada provides some guidelines for rooftop infrastructure, the uniqueness of articulating solar panels requires further support from experimental testing. This testing can be carried out both physically and virtually. Since this project was selected to look at wind forces, the physical testing would be best carried out in a wind tunnel, and the computational testing would be carried out using computational fluid dynamics software. Ideally, all simulations would be performed on a computer. This would save time and money, since allotting time in a wind tunnel and building an experimental apparatus is expensive. However, being able to test in a wind tunnel allows for validation of computer simulations, and performing these tests will allow greater confidence in future computer simulations.

\subsection{Research Goals}

Information is lacking regarding the stress a solar panel will place on a roof top due to a solar panel being mounted on it while exposed to the wind. Past aerodynamic studies of solar panel arrays have been performed, but are for limited operational cases. Shademan and Hangan (2009) [2] studied wind loading on solar panels at different elevation angles, wind directions and distances to nearby panels using Reynolds Averaged Navier Stokes computational experiments, simulating a single wind speed over flat, open terrain. The solar panels simulated were rigid and located at ground level. Geurts and Bentum (2007) [3] used wind tunnel and full scale experiments to find wind loads on rooftop solar panels at various locations on a rooftop, however these panels were of the flat, rigid nature. The goal of this thesis' research is to study the effect of wind on a solar panel mounted on the roof top of a large retail store. The research is aimed to understand the flow patterns that exist moving over the building and the solar panel situated on it, such as 
flow separation and vortex shedding frequency. It is desired that an understanding of the loads and torques on the panel be evaluated from wind tunnel testing and computational analogues. Although it is not expected that exact numerical values will be found to the extent that they may be scaled to full size models, it is hoped that magnitudes and trends in the data will provide a basis for narrowing down critical design cases that may be further examined in the future and refined to the point of correctly calculating the precise numerical loads and torques for full scale models. The test cases will be designed to replicate installation cases as well as operational cases that the solar panel may experience. With results from wind tunnel and computational analysis, the ultimate goal is to provide a basis for an organization to justify installation of a panel on their property that satisfies the legal requirements of National Building Code of Canada.

Before beginning the testing phase of this project, the National Building Code of Canada is presented in Chapter 2 to show what the current provisions are, and what is required of experimental testing. The fundamentals of fluid dynamics will be presented in Chapter 3 to provide an understanding of the wind dynamics present in the experiments, and will be followed by an examination in Chapter 4 of the environment that must be simulated, that of the earth's atmospheric boundary layer. This will form the basis for presenting the wind tunnel design and its setup in Chapter 5, followed by the presentation and discussion in Chapter 6 of the wind tunnel testing. These results will be discussed based on data trends, and future testing and considerations will be presented. Chapter 7 will present the setup of the computational simulation, with its results presented and discussed in Chapter 8. The thesis will conclude in Chapter 9 with a summary of what was accomplished, the significant contributions it has made to the body of wind engineering, and recommended future work. 


\section{Chapter 2}

\section{Roof Structures in the National Building Code of Canada}

The National Building Code of Canada provides up-to-date regulations that must be adhered to while designing rooftop structures. The code provides, among many other things, a validation for designing against wind loads on rooftops. This chapter will present the important considerations necessary to comply with the building code in regards to rooftop structures such as solar panels.

\subsection{Specified Loads}

The three categories of loads referenced in the national building code [4, s. 4.1.2.] that require consideration are the dead load, snow load, and wind load. These loads are defined as:

D dead load - Permanent load due to weight of the panel array structure and any structure it is supporting $[4$, s. 4.1 .4 .].

$\mathbf{S}$ snow load - Variable load due to weight of snow accumulation on the panel array structure 
W wind load - The load induced on the panel array structure due to the presence of wind forces

These loads may be increased from their minimum specified values to account for dynamic effects where applicable.

To determine the specified loads for $\mathrm{W}$, importance categories will assign different values to different buildings, as shown in Table 2.1 [4, s. 4.1.2.].

Table 2.1: Importance categories for buildings [4]

\begin{tabular}{|c|l|}
\hline Importance Category & Use and Occupancy \\
\hline Low & $\begin{array}{l}\text { Low hazard in the event of failure, i.e. mi- } \\
\text { nor storage buildings, etc }\end{array}$ \\
\hline Normal & $\begin{array}{l}\text { All buildings except those which are low, } \\
\text { high, or post-disaster }\end{array}$ \\
\hline High & $\begin{array}{l}\text { Disaster shelters, i.e. schools, community } \\
\text { centers, etc }\end{array}$ \\
\hline Post-disaster & $\begin{array}{l}\text { Essential services, i.e. hospitals, trans- } \\
\text { portation control, sewage treatment, etc }\end{array}$ \\
\hline
\end{tabular}

Ssince the focus of this thesis is wind loads on the rooftops of large retail buildings, normal importance will be assigned and the loading from the wind will be investigated.

\subsection{Wind Loads}

Three different procedures exist to determine the wind loads on buildings. The first is called Static Procedure. This is appropriate for cladding and in most other cases including low and medium rise building structures. Dynamic actions of the wind can be represented by equivalent static loads. 
The second procedure is called the Dynamic Procedure. This procedure is used to determine the overall effects of the wind, including resonance, and is primarily used for tall buildings and slender structures. The only difference between this and the Static Procedure is that the gust effect factor, $C_{g}$, and the exposure factor, $C_{e}$, are determine differently. $C_{g}$ is found using the wind turbulence intensity for the building site as a function of height and surface roughness of surrounding terrain, as well as building properties such as height, width, natural frequency, and damping. The gust effect factor is expected to give a static design pressure that represents the same peak load effect as the dynamic resonant response to the turbulent wind. [5, Commentary I]

The third procedure is called the Experimental Procedure. It consists of wind tunnel testing or other experimental methods, and can be used as an alternative to the Static and Dynamic Procedures. Buildings that may be subjected to buffeting or channelling due to upwind obstructions, vortexes, or aerodynamic instability are recommended to use the Experimental Procedure.

The specified external pressure due to wind on part or all of the surface of a building shall be calculated using the formula

$$
p=I_{w} q C_{e} C_{g} C_{p}
$$

where

- $p=$ specified external pressure acting statically and in a direction normal to the surface, either as a pressure directed towards the surface or as a suction

- $I_{w}=$ importance factor for wind load 
- $q=$ reference velocity pressure

- $C_{e}=$ exposure factor

- $C_{g}=$ gust effect factor

- $C_{p}=$ external pressure coefficient, averaged over the area of the surface

The wind load importance factor $I_{w}$ is found using Table 2.2 .

Table 2.2: Importance factor for wind load [4]

\begin{tabular}{|c|c|c|}
\hline Importance Category & ULS & SLS \\
\hline Low & 0.8 & 0.75 \\
\hline Normal & 1 & 0.75 \\
\hline High & 1.15 & 0.75 \\
\hline Post-disaster & 1.25 & 0.75 \\
\hline
\end{tabular}

The net wind load for the entire structure is the difference between the loads on the windward and leeward surfaces. This can also be done by taking the sum of the products of the external pressures and the areas acted upon [4, s.4.1.7.1.].

The reference velocity pressure $\mathrm{q}$ is based on the probability of being exceeded any one year over 50 years [4, s.4.1.7.1.].

The exposure factor $C_{e}$ varies for different terrain. For open terrain such as level terrain with only scattered buildings and trees, the exposure factor is $(h / 10)^{0.2}$. It is never lower than 0.9 ; where $\mathrm{h}$ is the reference height above grade in meters for the surface. For rough terrain, such as suburban, urban, or wooded terrain, the exposure factor is $0.7(h / 12)^{0.3}$. It is never lower than 0.7 ; where a change in roughness upwind is uninterrupted for at least $1 \mathrm{~km}$ or 10 times the building height, whichever is greater. For intermediate cases between the two exposures and cases where the upwind roughness is interrupted, an appropriate interpolation method can be used. 
If a dynamic approach to the action of wind gusts is used, an appropriate value is used depending on both height and shielding. [4, s.4.1.7.1.]

The gust effect factor, $C_{g}$, for main structural members is 2.0. For a dynamic approach, the gust effect factor is a value that is appropriate for the turbulence of the wind and for the size and natural frequency of the structure. [4, s.4.1.7.1.]

If the height of a building is greater than four times its minimum effective width, or greater than $120 \mathrm{~m}$, or if the building is light weight, low frequency and low damping properties make the building susceptible to vibration. This shall be designed by experimental methods for the danger of dynamic overloading, vibration and the effects of fatigue or by using the dynamic approach to the action of wind gusts. [4, s.4.1.7.2.]

The building and structural members must be capable of withstanding the effects of the full wind load acting on each of the two principal horizontal axes considered separately. This must also be true if $100 \%$ of the load is removed from any portion of the area. The wind loads must also be sustained simultaneously at $75 \%$ of their full value, and with the added capability to withstand $50 \%$ of these loads removed from any portion of the area. [4, s.4.1.7.3.]

\section{Vortex Shedding}

Slender structures should be designed to resist the effects of von Karman vortex streets. A slender structure is defined by a length to width ratio exceeding five. Vortexes will be shed alternatively from one side and then the other along the length of the structure. This will produce a fluctuating force at right angles to the wind direction. [5, Commentary I] The critical mean wind speed when resonance occurs due to vortex shedding is: 


$$
V_{H c}=\frac{1}{S} f_{n} D
$$

where $\mathrm{S}$ is the Strouhal Number based on the cross section shape, $f_{n}$ is the frequency in $\mathrm{Hz}$, and $\mathrm{D}$ is the width. For non-circular cross sections the Strouhal number is about $1 / 7$.

\subsubsection{Calculating Wind Effects}

Structures need to be designed to ensure that main and secondary structures can withstand the pressures caused by the strongest wind blown at a certain location in many years. Flexible structures also need to be designed to minimize the excess oscillations caused by wind.

The wind acting upon a structure is treated as both a time-averaged component and as a gust/unsteady component. For small structures that are completely enveloped by wind gusts, only the peak gust velocity needs to be considered. A gust factor may be incorporated which varies for types of structures and the size of the area over which the pressure acts. [6, App.C Div.B]

The wind speeds used in the building code are regionally representative, one hour averages, at a $10 \mathrm{~m}$ height in flat open terrain based on long-term wind records that have been observed at a large number of weather stations across Canada.

The wind velocity pressure, $\mathrm{q}$, is calculated using the following equation

$$
q=0.5 \rho V^{2}
$$

where $\rho$ is the average air density for the windy months of the year and $V$ is the wind 
speed in meters per second. An average value of $1.2929 \mathrm{~kg} / \mathrm{m}^{3}$ for air density (from the building code) was used even though density depends on both air temperature and atmospheric pressure. This value is within $10 \%$ of the monthly average of air densities. [6, App.C Div.B]

\subsection{Structural Equivalents}

Design methods not specified in Part 4 of the building code are permitted. Such methods include full-scale testing and model analogues. This provision is used to permit the acceptance of innovative structures or for model testing of structural behaviour due to snow or wind loads. The level of safety and performance must be at least equivalent to that provided by the design in Part 4 of the building code. It also requires that loads and designs conform to Section 4.1 of the building code. The designer must prove to the appropriate authority that the structure provides the level of safety and performance required. This can only be established by analyzing the structure for the loads set out in Section 4.1 of the building code and by demonstrating that the structure at least meets the requirements of the design standards outlined in Sections 4.3 and 4.4 of the building code. [6, sA-4.1.3.2(2)]

Since the dual-axis solar tracker is susceptible to vibration and is subjected to vortexes and aerodynamic instability, the structure must be designed by experimental methods. 


\section{Chapter 3}

\section{Fluid Dynamics Background}

The two properties examined are the forces/moments on the panel submerged in wind flow, and the frequency of vortex shedding that can be expected from the panel. This chapter will present the causes of these properties.

\subsection{Fluid Forces and Moments on Flat Plates}

Plates at incidence angles of less than 90 degrees will have a force acting on it that is tangential to the surface due to skin friction. This tangential force when compared to the normal force of the flow is insignificant for incident angles that are 10 degrees and higher. The distance between a plate and a surface will affect both the forces acting on the plate, and the location of the center of pressure. [7]

Experiments have shown that the normal force coefficient for rectangular plates does not vary significantly with the Reynolds number, when in ranges between $10^{3}$ and $3 \times 10^{6} .[7]$

Approximating for thin plates is valid for cases when the plates have blunt edges, and the maximum thickness of the plate is about $0.2 \mathrm{c}$, where $\mathrm{c}$ is the panel chord length. Meeting these conditions renders the effect of the thickness on the normal force coefficient insignificant. [7] 
For plates mounted near a surface, such as a panel on a rooftop, the flow velocity will vary with distance from the surface to the panel. Such a plate can also be immersed in shear flow. The effective mean velocity in such a flow is defined by:

$$
V_{e f f}^{2}=\frac{1}{2} \int_{1}^{2} V_{h}^{2} d h
$$

where the limits represent the bottom and top of the plate. The normal force in a shear flow is thus given by:

$$
N=\frac{1}{2} \rho V_{e f f}^{2} S C_{N}
$$

where $C_{N}$ would be the force coefficient applied during a uniform flow.

For plates that are inclined to the flow, the plate thickness becomes more restrictive. Thicknesses exceeding $0.05 \mathrm{c}$ cannot be assumed for flat plate approximations at low incidence angles. As the angle of a flat plate changes, the location of the center of pressure will also change. [7]

\subsection{Forces on Panels Mounted in Turbulence}

Turbulent shear flow on surface mounted prisms induces mean forces and moments. Other excitation methods such as vortex shedding should be investigated when dealing with applications such as wind around a roof mounted panel. The velocity profile of the approaching flow, its turbulence intensity and scale, flow incidence, and edge radius are corrected for by special factors. [8]

The mean force acting on a body in the $X$ direction is given by the following formula: 


$$
F_{x}=\frac{1}{2} \rho \bar{V}_{H}^{2} b H C_{F_{x}}^{\prime} k_{s} k_{l} k_{\imath} k_{\phi} k_{r}
$$

where $C_{F_{x}}^{\prime}$ is the force coefficient for low turbulence uniform flow over sharp edged prisms at zero incidence. The coorinate system used in the wind tunnel can be found in Figure 5.15. The remaining variables are $\mathrm{k}$ factors that correct the flow velocity profile turbulence scale and intensity, incidence, and edge radius. [8]

Correction for Shear Flow, $k_{s}$

The variation of the mean flow velocity with length characterizes the factor $k_{s}$, and can thus be related to a surface roughness parameter. An analytical expression for $k_{s}$ is: $k_{s}=\frac{1}{2 \alpha+1}$, where $\alpha$ represents the terrain roughness. [8]

Corrections for Turbulence, $k_{l}, k_{\imath}$ Non-uniform velocity profiles tend to reduce the mean force, while turbulence increases the mean force. When estimating design wind loads this must be taken into account due to the atmospheric wind's turbulent nature. $k_{l}$ accounts for the effects of turbulence scale. $k_{\imath}$ accounts for the effects of turbulence intensity. Variations of $k_{l}$ are consistent with variations in $k_{\imath}$ with the ratios of length to thickness and chord to thickness. [8]

\section{Corrections for Flow Incidence, $k_{\phi}$}

This factor allows for the effect of flow incidence on the mean force of sharp-edged panel prisms. Flow separation and reattachment on the side, bottom and top faces of the prisms are characteristics that $k_{\phi}$ captures with changes in length to width and height to width ratios. [8]

\section{Corrections for Rounded Edges, $k_{r}$}

Flow separation and reattachment are affected by the roundness of the edges. Forces generally decrease with an increase in relative roundness. [8] 
Mean $Z$ Force is given by

$$
F_{z}=\frac{1}{2} \rho \bar{V}_{H}^{2} c H C_{F_{z}}^{\prime} k_{s} k_{l} k_{\imath} k_{\phi} k_{r}
$$

It is derived from the value of the $\mathrm{X}$ force with an incidence angle of $90-\phi$ to the oncoming flow, and by interchanging the thickness $\mathrm{b}$ with the chord c. [8]

Mean $Y$ Force is given by $F_{y}=\frac{1}{2} \rho \bar{V}_{H}^{2} c b C_{F_{y}}$. Turbulence is likely to reduce $C_{F_{y}}$ compared to smooth flow when the length to width ratio is greater than unity. The mean $\mathrm{Y}$ force is usually small due to the symmetry of a prism panel. [8]

\subsection{Understanding Vortex Shedding}

Vortex shedding in the wake of an object immersed in a fluid flow can cause dynamic loading on structures and can induce vibrations. Inclined flat plates with sharp edges have had their vortex shedding frequency studied in detail. The Strouhal number is a non-dimensional shedding frequency based on the flow velocity and structure characteristic length. When the Strouhal number is based on the chord of the plate and the free-stream velocity, it is insensitive to changes in Reynolds numbers above 1.1-3.2 $\times 10^{4}$. [9] Two dimensional flat plates can theoretically be treated as a straight line without any thickness [9], instead of a solid structure with edges. Furthermore, additional studies have shown that flat plate Strouhal numbers are essentially independent of the angle of attack above 30 degrees. Below 25 degrees there is a significant rise in the Strouhal number. This trend is displayed in Figure 3.1 shown next: 


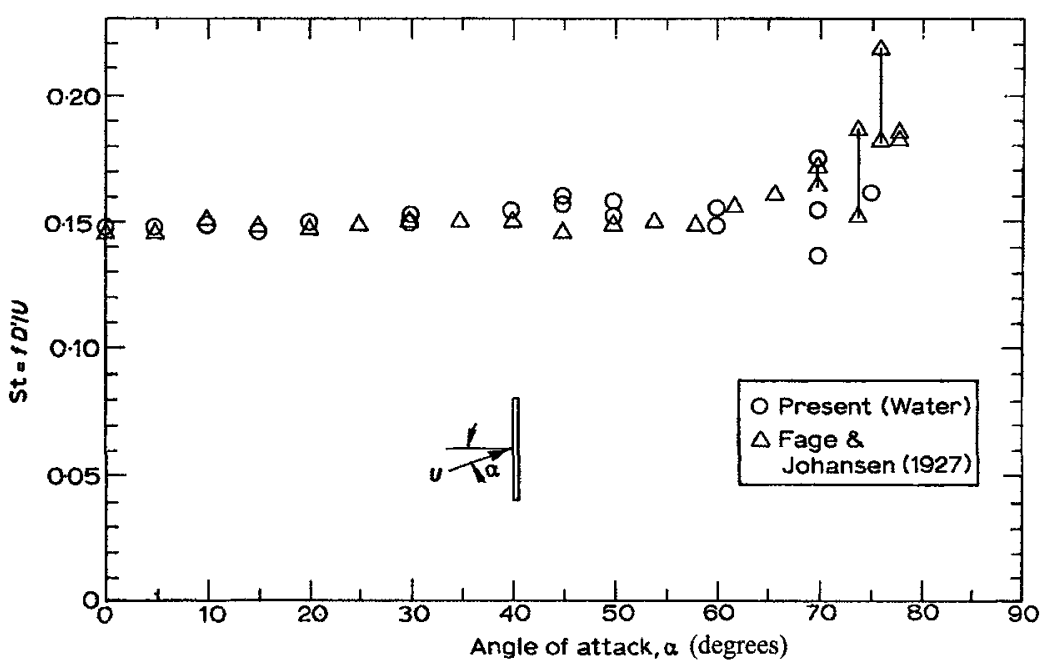

Figure 3.1: Strouhal number of flat plate at incidence $[9,10]$ 


\section{Chapter 4}

\section{The Atmospheric Boundary Layer}

The effects of the atmospheric boundary layer on wind speed can be estimated reasonably accurately when the ground roughness and mean-wind field are known. The properties of the mean-wind field of major interest are velocity extremes and recurrence intervals. [11]

\subsection{Origin of the Wind}

The nature of wind can be traced back to the sun. Solar radiation from the sun heats the planet differentially. Locations closer to the equator receive much higher doses of solar radiation, and locations closer to the poles receive less. Since the surface of the planet will thus have differential temperatures, gradients of pressure will be created in the atmosphere. Wind is created high above the earth's surface by the rotation of the earth modifying the differentially heated air in the atmosphere. The gradient wind velocity occurs at heights greater than about 1000 feet. This is where the frictional forces near the ground no longer influence the wind velocity. The friction of the earth's surface along with obstructions such as trees and buildings cause drag on the airflow and subsequent shear forces (Reynolds stresses) create a velocity profile for a boundary layer, with zero velocity at the earth's surface and maximum velocity 
at the top of the layer. Drag forces at the ground surface slow down the airflow, which results in an exchange of momentum transmitted upwards between layers due to turbulence. Therefore, the wind velocity is dependent on the geographic location, the nature of the terrain, and the height above the ground. [11]

\subsubsection{Isotropic Turbulence and Parallel Shear Motion}

In a wind tunnel, or in large regions of the atmosphere, the mean values of flow velocity are "slowly variable functions" of time, which means that fluctuations are so fast that variations from the mean value are negligible. [12]

The mechanism behind turbulent friction is theorized to be from either a transfer of momentum or a transfer of vorticity. It is known however that turbulence development depends on balance between energy transfer of mean motion into fluctuations and dissipation due to the fluctuations. Inertia and viscosity terms should balance out at large Reynolds numbers. The dissipation in every layer of fluid is equal to the work done by the shearing stress. [12] This provides a basis for understanding the turbulent force at work in the atmospheric boundary layer.

\subsubsection{Mean Flow in Atmospheric Boundary Layer}

Viscosity plays an important part in controlling the rate of the dissipation of turbulence between layers of wind. The region of influence below the gradient height is the atmospheric boundary layer. In this region, the wind velocity profile can be accurately defined by the Prandtl logarithmic profile; this agrees with experimental wind tunnel measurements, as well as over natural surfaces. A more simple power law profile representation can be used as shown below: 


$$
\frac{V_{Z}}{V_{G}}=\left(\frac{Z}{Z_{G}}\right)^{\alpha}
$$

The value for $\alpha$ and $Z_{G}$ vary for different natural surfaces, and must be measured. Many geographical locations have been measured, varying from open water to the center of a large city. Profiles can be simplified into three types of terrain; open, wooded country or suburban, and urban. This transition is seen in Figure 4.1.

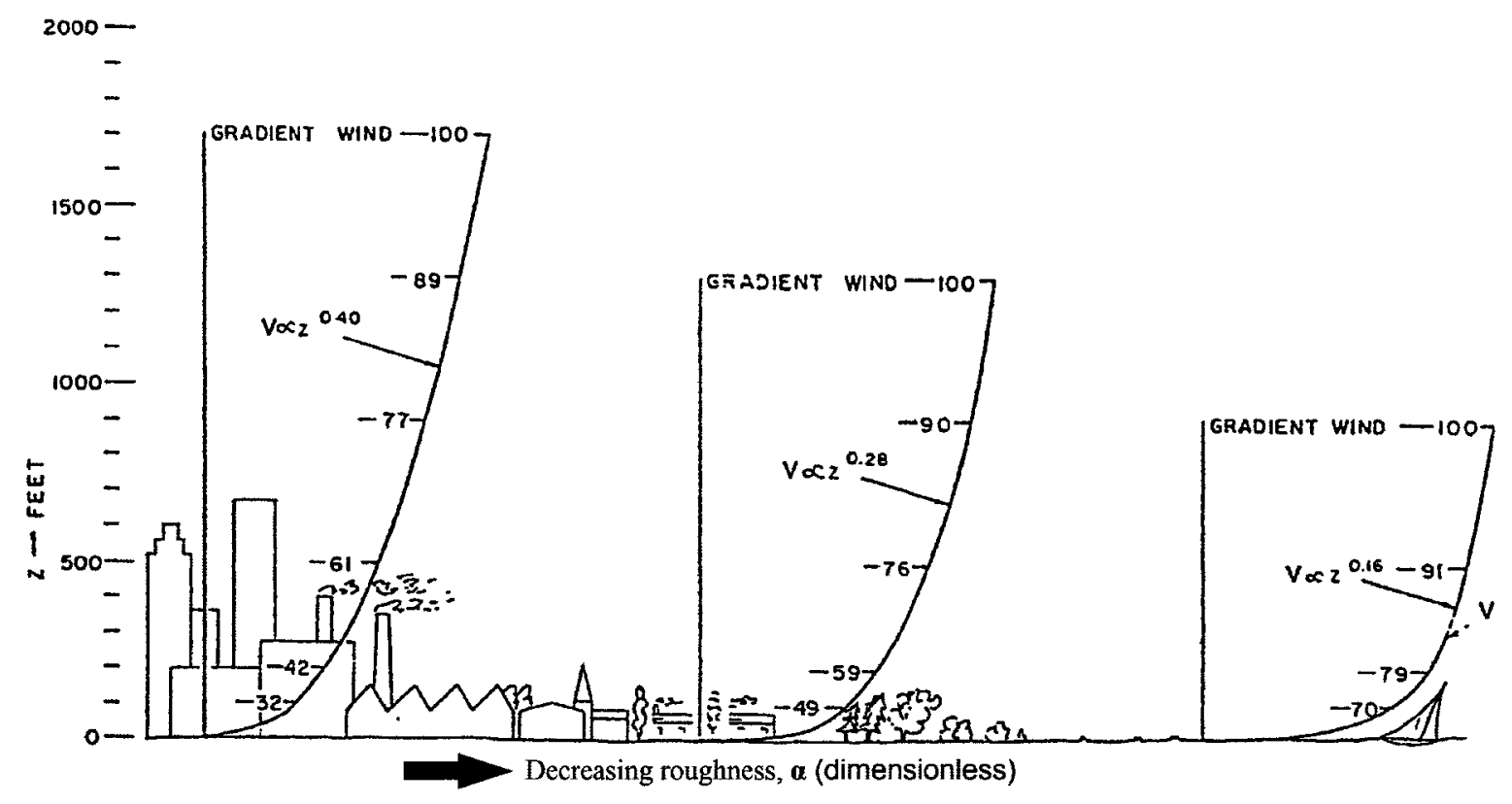

Figure 4.1: Profiles over differing terrain roughness [11]

It is important to understand how far much downwind distance is required of a change in surface roughness to be considered a new type of terrain. This is called the fetch distance, and is demonstrated in Figure 4.2. It takes about twice as far for a wind to pick up speed over a smoother surface than it takes for a wind to slow down over a rougher surface. The roughness of an urban area can slow the wind down to a third of wind coming from open country. 


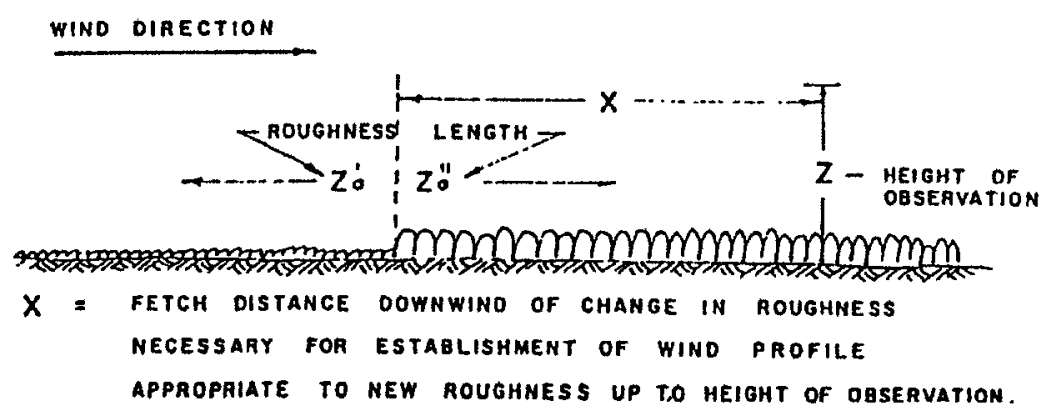

Figure 4.2: Distance needed to establish new wind profile after roughness change [11]

\subsection{Determination of Design Wind Velocities}

The wind causes one of the principal loads acting on above ground structures. Determining this force is fundamental to both the safety and the economic impositions to overcome. It is important to know the extreme sustained wind velocity as it will create the steady component of pressure. For locations of differing surface roughness, design wind velocities of given probability of occurrence can be predicted. [13] The gradient velocity is the velocity of the free air, and can be found from

$$
V_{G}=r w \sin \lambda\left[\sqrt{\frac{\frac{d p}{d N}}{\rho p r w^{2} \sin ^{2} \lambda}+1}-1\right]
$$

where $r$ is the isobar radius of curvature, $w$ is the earth's rotational speed, $\lambda$ is the latitude, $d p / d N$ is the pressure gradient, and the density of the air is $\rho$.

Wind velocity increases with height. The most widely used profile to express this increase, for structural purposes, is the exponential profile previously discussed due to its simplicity. Surface roughness is not defined as individual obstructions but rather the cumulative statistical drag effect of numerous wind obstructions. [13]

Based on qualitative descriptions of terrain, wooded countryside, parkland, towns, 
and outskirts of large cities, the suburban terrain in this study can be shown in Figure 4.3 to correspond to a power-law exponent of $\alpha=0.28$.



Figure 4.3: Increase of velocity with height over different terrain [13]

This refers to mean wind velocities over level ground, the presence of a large scale severe storm, and to heights between $30 \mathrm{ft}$ and the gradient velocity. [13]. The boundary layer shape varies according to the terrain factor $\alpha$, which can be found in Table 4.1. 
Table 4.1: Coefficients for boundary layer shape [14]

\begin{tabular}{|l|r|r|r|}
\hline Terrain & $\mathrm{h}(\mathrm{ft})$ & $\mathrm{h}(\mathrm{m})$ & $\alpha$ \\
\hline Open country & 280 & 85.3 & 0.15 \\
\hline Wooded and suburban & 1200 & 365.8 & 0.28 \\
\hline Urban & 1700 & 518.2 & 0.4 \\
\hline
\end{tabular}

\subsection{Response of a Structure to Wind}

For structural design, two properties of the wind are required; namely the structure of the wind, and the statistics of the wind climate. To define the structure of the wind, the mean velocity profile is required, along with the properties of its turbulence. As discussed earlier, a power law velocity profile was both simple and adequate under conditions over all type of terrain so long as the terrain was relatively level.

The gradient velocity is useful as a reference wind speed because it is independent of local terrain roughness. More theoretical profiles depend on the Rossby number, making the profile slightly dependent on the latitude. It has been found that these differences are not significant. [15]

Relating back to the power law profile during transition from an old surface to a new surface, it is useful to understand the fetch required to grow the mean velocity profile that is characteristic of the new surface. Significant differences in velocity are mostly in the first $500 \mathrm{ft}$. To produce most of the significant changes to the profile, a mile downwind of the change in roughness should be sufficient. [15] 


\subsubsection{Static Analysis of a Rooftop Structure}

Building test programs perform static wind loads, which may be used later for dynamic tests. If the atmospheric boundary layer is unknown, then the maximum wind speed at $30 \mathrm{ft}$ altitude is used to construct a boundary layer according to

$$
\frac{V_{Z}}{V_{r e f}}=\left(\frac{Z}{Z_{r e f}}\right)^{\alpha}
$$

Work has been performed on similar roof top structures, namely solar collectors [11]. Similarly though it is critical to measure loads and moments for a full range of azimuth. Loads are needed in a rooftop array to size the motors that swivel the pitch of the array, whereas the panels will also need to have motors sized for the yawing motion. Such a program requires force and moment data. Force and moment are used to determine loads on the array foundation, which is a rooftop. Array spacing is additionally varied, with runs at different wind speeds to confirm the lack of variation in Reynolds number. Dimensionless parameters can be found as shown below.

$$
\begin{gathered}
C_{\text {force }}=\frac{\text { Force }}{q S} \\
C_{\text {moment }}=\frac{\text { Moment }}{q S c}
\end{gathered}
$$

Data is usually collected as plots of drag, lift, and moment against different elevation and azimuth angles. It has been found that azimuth angle does not produce large changes in forces and moments. [14] 


\subsection{Designing an Atmospheric Boundary Layer}

Non-aeronautical uses of wind tunnels are routinely conducted by wind engineers to study interactions of the wind from the atmospheric boundary layer with that of structures on the ground. A wind tunnel that is used to simulate the natural boundary layer over a structure is labelled a Meteorological Wind Tunnel. Check runs at varying wind speeds should be performed to make sure that Reynolds number effects are small. The Reynolds numbers for buildings are based on the width of the building. The distribution of the atmospheric boundary layer is also required to have a proper velocity distribution. The boundary layer must be scaled to match the scaled down buildings. Modern wind tunnels attempt to duplicate this distribution by placing spires upstream of the wind flow, followed by roughness elements running 10-15 test section heights. A turntable is often implemented to mount the urban building complex, with rotations of the turntable representing wind speeds from varying directions. Temperature gradients do not need to be simulated because when the wind blows intensively, the air is mixed up to the point that temperature is largely homogeneous. [14] In general a model building is scaled between 1 to 50 and 1 to 500 . For this study it is 1 to 100. Simple formulas have been developed to design spires and surface roughness of wind tunnels such that they can generate a boundary layer that represents that of the planet. Figure 4.4 illustrates this setup.



Figure 4.4: Wind tunnel spires and roughness elements [16] 
This knowledge was discovered in the 1960's and has been developed to produce the same velocity profiles with the right large scale turbulence and intensity that match the atmospheric boundary layer. The roughness elements are meant to create the correct velocity profile, while the spire acts to create large scale eddy turbulence from vortexes shedding of the edges. It has been found that turbulence properties and the mean velocity profile downstream of the spires are sensitive to the triangular shape of the spires, meaning they must be relatively triangular shape. The correct drag is maintained by keeping the triangular spires as flat plates, creating large pressure drag and large turbulent vortex structures peeling of the triangle's edges. Distributed roughness to create floor friction also assists in developing the boundary layer, specifically near the floor. [16]

When it is assumed that the flow upstream of the spires is uniform, the power law is defined as

$$
\frac{U_{Z}}{U_{\delta}}=\left(\frac{Z}{\delta}\right)^{\alpha}
$$

where $U_{Z}$ is the velocity at height $Z, U_{\delta}$ is the velocity at $Z>\delta$, and $\alpha$ is the power law exponent. An expression can be created for the total frontal area of the spires to produce a boundary layer with a required $\alpha$ and $\delta$. The expression uses the spire height $\mathrm{h}$ and spire base length $\mathrm{b}$. A distance of $6 \mathrm{~h}$ is required to ensure lateral homogeneity of the flow when the spires are spaced $\mathrm{h} / 2$ from each other's centerline. [16]

The height of the spire is found to be $h=\frac{1.39 \delta}{1+\frac{\alpha}{2}}$, whereas the ratio for the base to height is obtained by $\frac{b}{h}=0.5\left[\gamma \frac{H}{\delta} 1+\gamma\right]\left(1+\frac{\alpha}{2}\right)$, where $\gamma=\beta\left[\frac{2}{1+2 \alpha}\right]+\beta$ and $\beta=\left(\frac{\delta}{H}\right) \frac{\alpha}{1+\alpha} . \mathrm{H}$ is the height of the test section. The test section should be a multiple of $\mathrm{h} / 2$. The surface friction required by the roughness elements is 


$$
C_{f}=0.136\left[\frac{\alpha}{1+\alpha}\right]^{2}
$$

To achieve the correct roughness size for the required surface friction, the ratio of the roughness height $\mathrm{k}$ to boundary layer thickness $\delta$ is

$$
\frac{k}{\delta}=\exp (2 / 3) \ln (D / \delta)-0.1161\left[\left(2 / C_{f}\right)+2.05\right]^{1 / 2}
$$

where $\mathrm{D}$ is the spacing of the roughness elements. These are the design principles that are used in the wind tunnel at Carleton University to create the atmospheric boundary layer that is used in this thesis study. 


\section{Chapter 5}

\section{Wind Tunnel Setup}

\subsection{Wind Tunnel and Test Configurations}

This section will describe the design, construction, and implementation of the wind tunnel experiment. It will first describe the characteristics inherent to the wind tunnel, and then the details of the test section. The array of test configurations will then be illustrated, followed by a description of how the data acquisition unit works.

\subsubsection{The Wind Tunnel}

The wind tunnel at Carleton University is classed as a low-speed, open-loop tunnel. The top free stream velocity that can be produced is approximately $60 \mathrm{~km} / \mathrm{h}$. However the large cross section and long length make it ideal for testing atmospheric flows, since it allows adequate distance for the build-up of the atmospheric boundary layer. At four inches above the tunnel floor, the turbulence intensity is measured at 12

To create the atmospheric boundary layer, four large spires are inserted into the flow far upwind of the test section, as shown in Figure 5.1. 


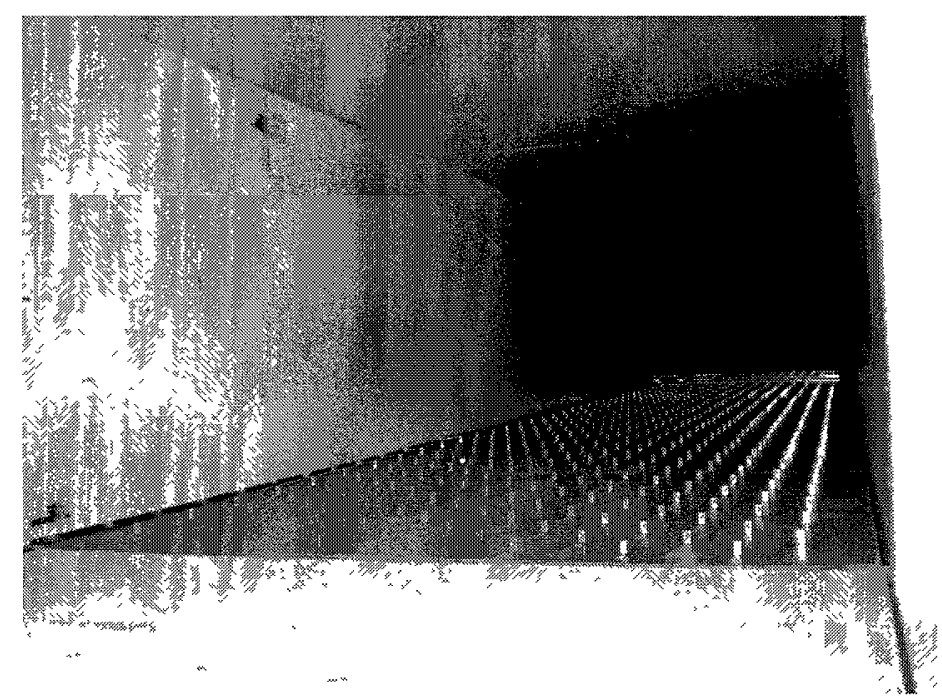

Figure 5.1: Upwind view from test section

Downwind of the spires, the tunnel floor is lined with roughness elements. These elements continue until the test section, which is shown at the end of the tunnel just before the fan in Figure 5.2.

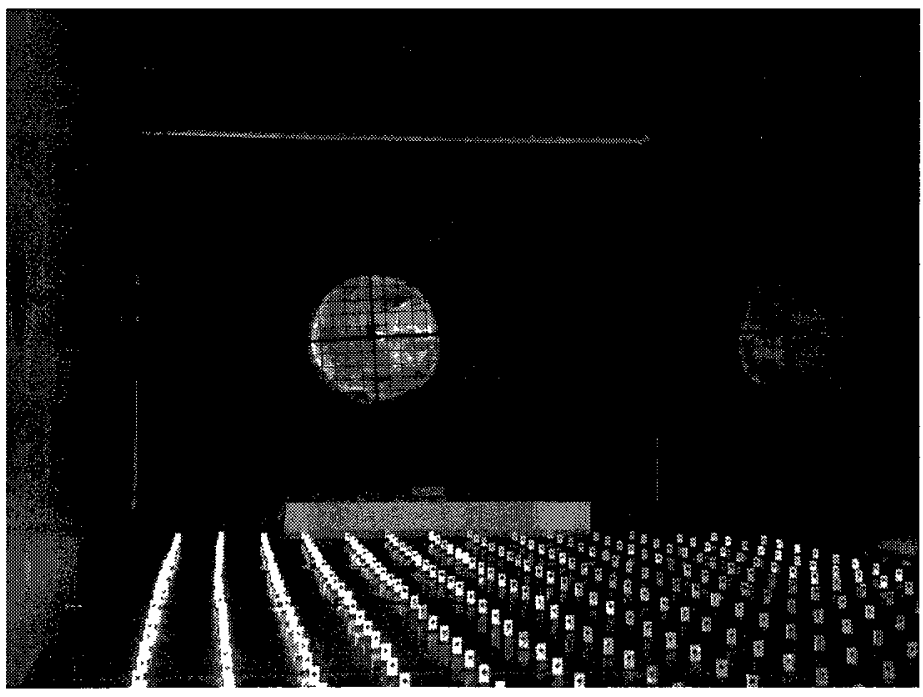

Figure 5.2: Downwind view of test section

A diagram of the wind tunnel with its relevant dimensions can be found below in Figure 5.3 




Figure 5.3: Wind Tunnel Diagram

\section{Creating an Atmospheric Boundary Layer}

A power law profile coefficient $\alpha$ and a boundary layer thickness $\delta$ can be used to represent the atmospheric boundary layer. To create this boundary layer in a wind tunnel, triangular spires are installed upstream of the experiment and roughness elements are distributed on the tunnel floor. [17] The power law coefficient $\alpha$ is related to the effects of surface roughness, and is used in the following form:

$$
\frac{U_{Z}}{U_{\delta}}=\left(\frac{Z}{\delta}\right)^{\alpha}
$$

The spire dimensions are determined from empirical relationships to produce the designed boundary layer, using Equations 4.7 and 4.8. The four spires are constructed from 3/4 inch plywood and are screwed to a two-tiered base also constructed from $3 / 4$ in plywood. Each spire has a base that is five inches wide, and 33 inches tall.

Roughness elements, spaced eight inches apart, added downstream from the spires will promote boundary layer growth. The elements are 1 inch by 1 inch L-brackets made of $1 / 8$ inch thick steel and are glued to the tunnel floor upstream of the test section. [17]

The test section will be described in further detail in the following subsection. 


\subsubsection{The Test Section}

Just before the fan at the end of the wind tunnel is the test section. The test section is accessible on either side of the tunnel via plexiglass doors that swing up and open. The floor of the test section contains a circular cut-out that can house various discs for different experiments. For the purpose of this thesis, a disc is inserted into the floor that has a square building mounted on top of it. This can be seen in Figure 5.4.

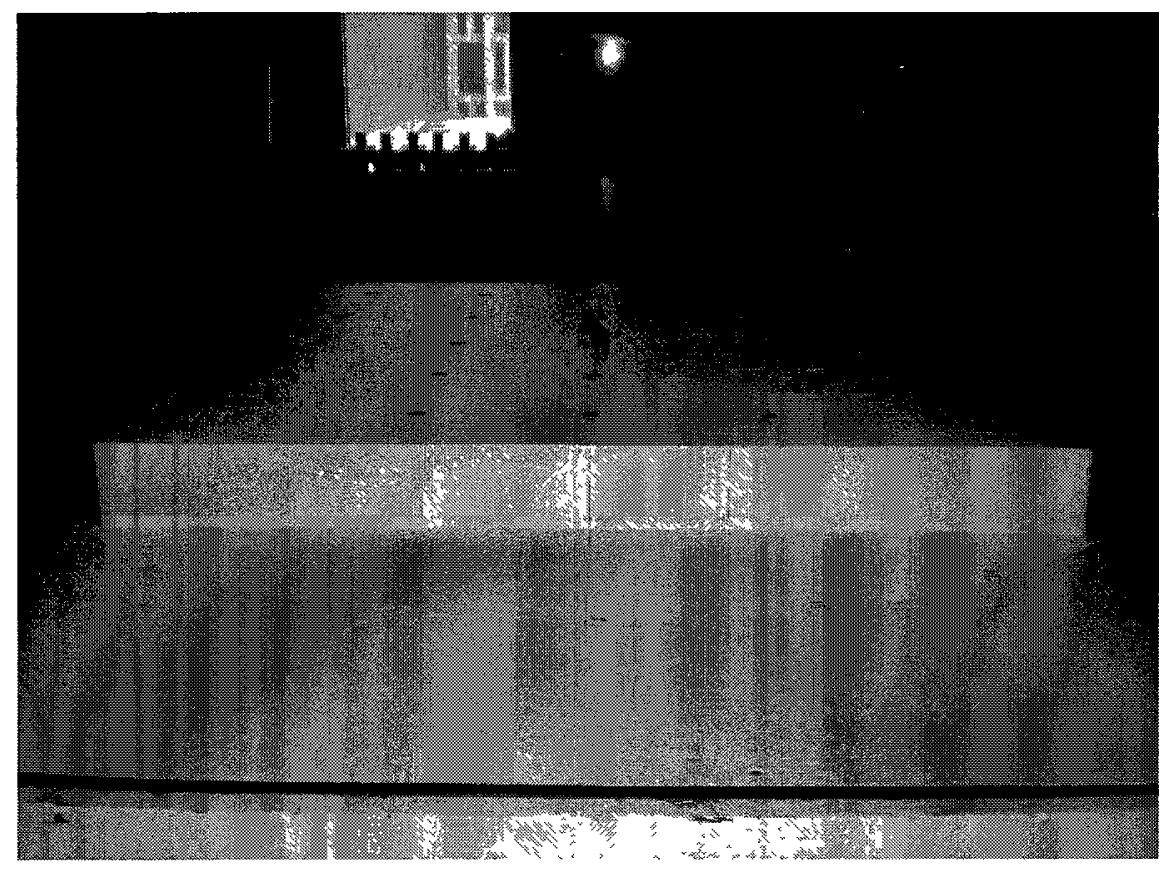

Figure 5.4: Test section initial panel setup

The square building has 25 holes across the roof surface uniformly distributed, with 25 holes allowing for adequate azimuth rotation space of each panel, as well as enough data points to allow for possible trends to be identified. Solar panel models are inserted into these holes. When the holes are not in use, plastic plugs are used to seal the openings. Scaled to approximately 1:105, the building's measures three inches tall and 32 inches wide, with each panel being 3.692 inches wide with a 1.488 inch chord. To match the Reynolds number of the scaled of the models fit into the 
wind tunnel, wind speeds many magnitudes higher that what is possible are required. As stated earlier, the force and moment coefficient results are insensitive to changes in Reynolds number for the given operating ranges. The building is capable of rotating 360 degrees on the wind tunnel floor. This allows for different wind directions to be simulated. The building is mounted to the disc using metal brackets. The disc is clamped to the wind tunnel floor from underneath the floor.

The building is completely hollow, which allows room for the load cell to be positioned under any of the holes in the roof surface. The load cell force platform can be seen in its position underneath the building in Figure 5.5.

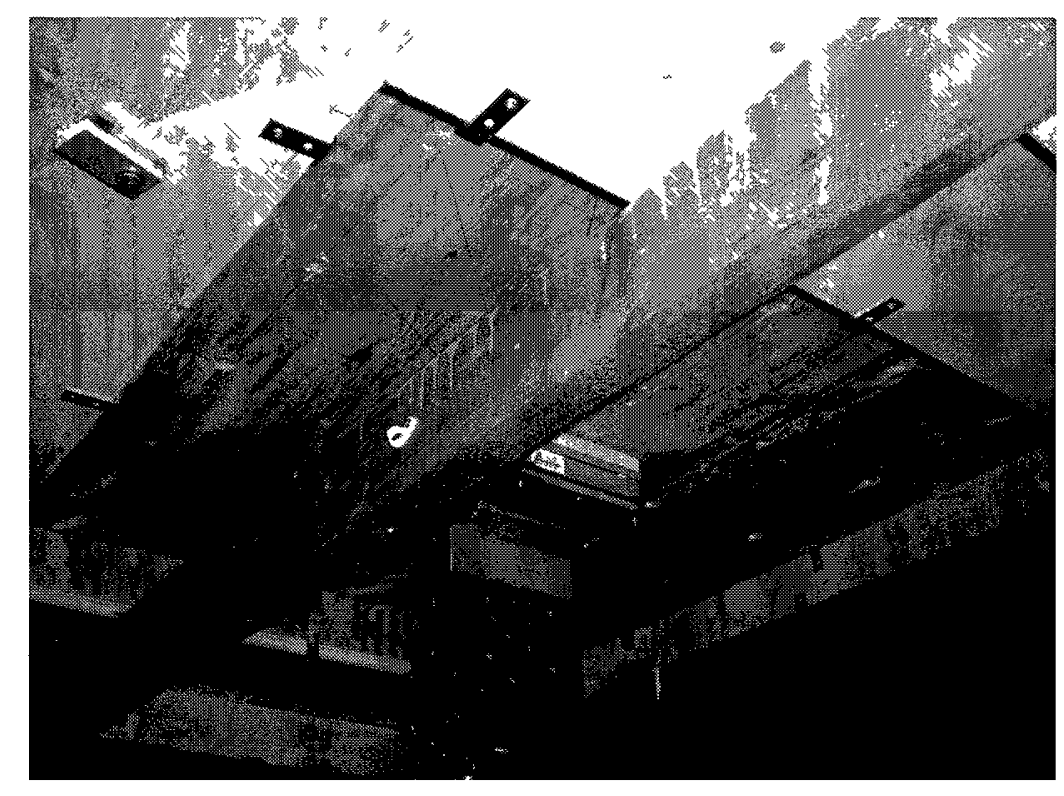

Figure 5.5: Load cell setup underneath building

The load cell is mounted to the top of a tripod that sits underneath the wind tunnel floor. The load cell is first mounted onto the tripod, and can then be raised up inside the hollow building underneath the roof. A side view of the test section, shown in Figure 5.6, shows the setup of the tripod in relation to the building. 


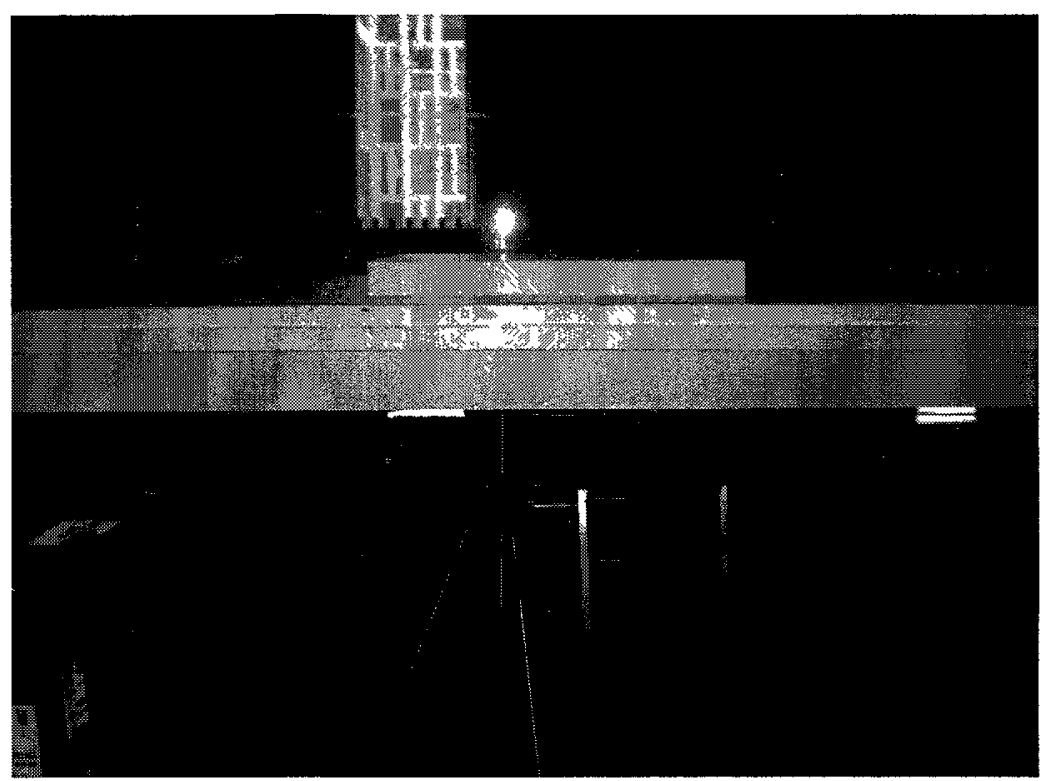

Figure 5.6: Side view of experiment setup

Thus far, the functions of the experiment in the test section have been described, but the description of how the experiment trials will actually be carried out must still be presented, and is shown in the next subsection.

\subsubsection{Test Configurations}

The tests were designed to investigate various parameters that could be changed in a full scale working model. The parameters that needed to be investigated were the effects of wind direction, wind speed, the panel azimuth angle, the panel elevation angle, the location of the panel on the rooftop, the proximity to another nearby solar panel, and the immersion of the panel in various panel field sizes. The layout of the rooftop and its panel locations are shown in Figure 5.7 below. 




Figure 5.7: Rooftop layout

The wind speed is changed simply by increasing the wind tunnel fan speed. The wind tunnel computer controls the fan by monitoring a pressure transducer and accepting user input via a wind velocity toggle switch.

To change the wind direction, the building and turn table disc are rotated in intervals of 15 degrees. Figure 5.8 shows the wind angled 45 degrees at the building. 


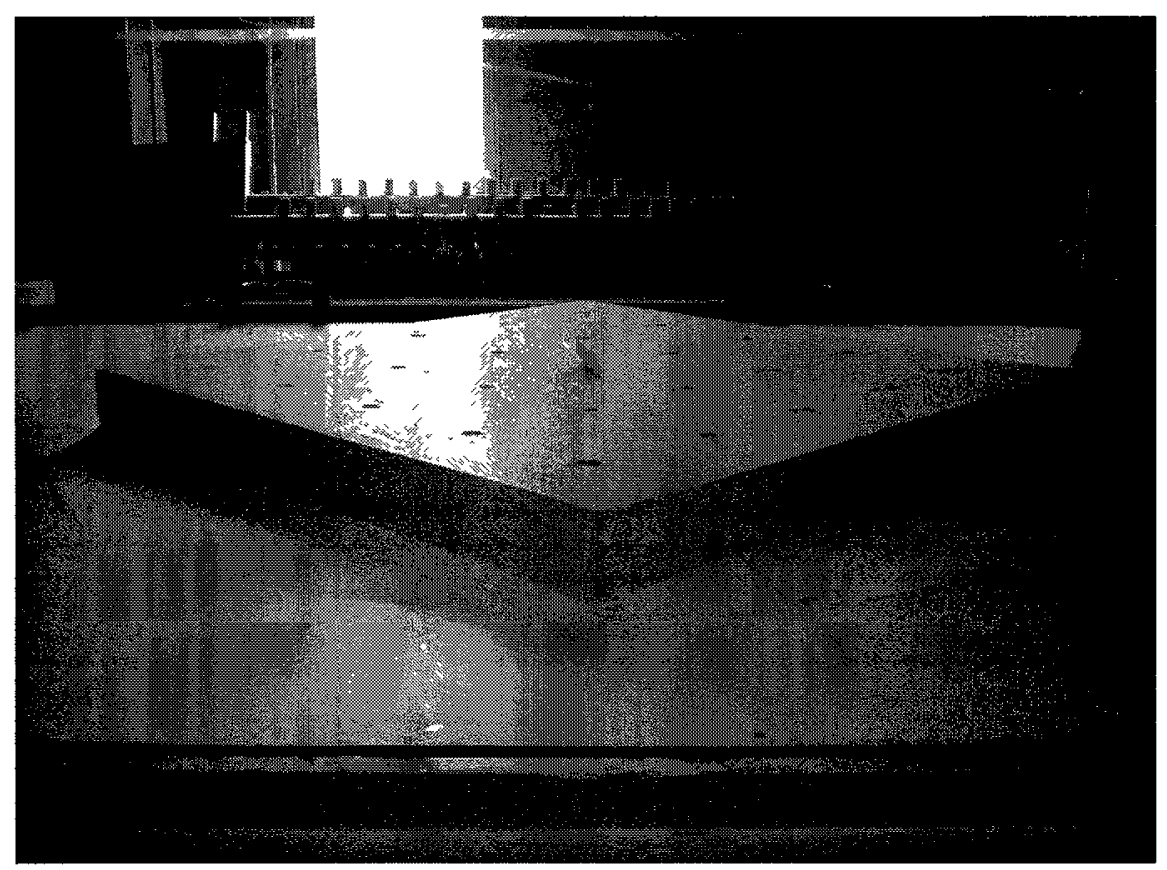

Figure 5.8: Changing wind direction

This configuration kept the panels oriented straight into the wind while the wind approaches the building from various angles. A similar configuration occurs when the wind direction remains constant on the building, but the solar panel rotates. This mimics a real panel's ability to track the sun's azimuth angle. The setup is designed to allow the panel to yaw a full 360 degrees.

The full scale solar panels are able to track the elevation of the sun in the sky. This changes the angle of the panel with respect to the horizon. Instead of creating a mechanical model, four different elevation angles were selected to test, and a model made from Acrylonitrile butadiene styrene (ABS) was constructed for each angle. These models can be seen in Figure 5.9. 




Figure 5.9: Panel angles

As can be seen, the four models represent 15, 30, 45, and 60 degree angles from the horizon. The panel can also change its location on the roof top. The effect of moving the panel closer to the building's leading, trailing, and side edges is thus monitored.

Applications for such solar panels may not be limited to a single panel on a roof top. Multiple panels could be present. The distance between two panels could have an effect on the flow around each panel. To measure this effect, while the main panel model remains at the roof top center, a second "dummy" panel is fixed to the roof. This dummy panel is moved forward, backward, and sideways of the main panel, while the flow effects were measured by the load cell on the main panel. An example of the dummy panel forward of the main panel is shown in Figure 5.10. 


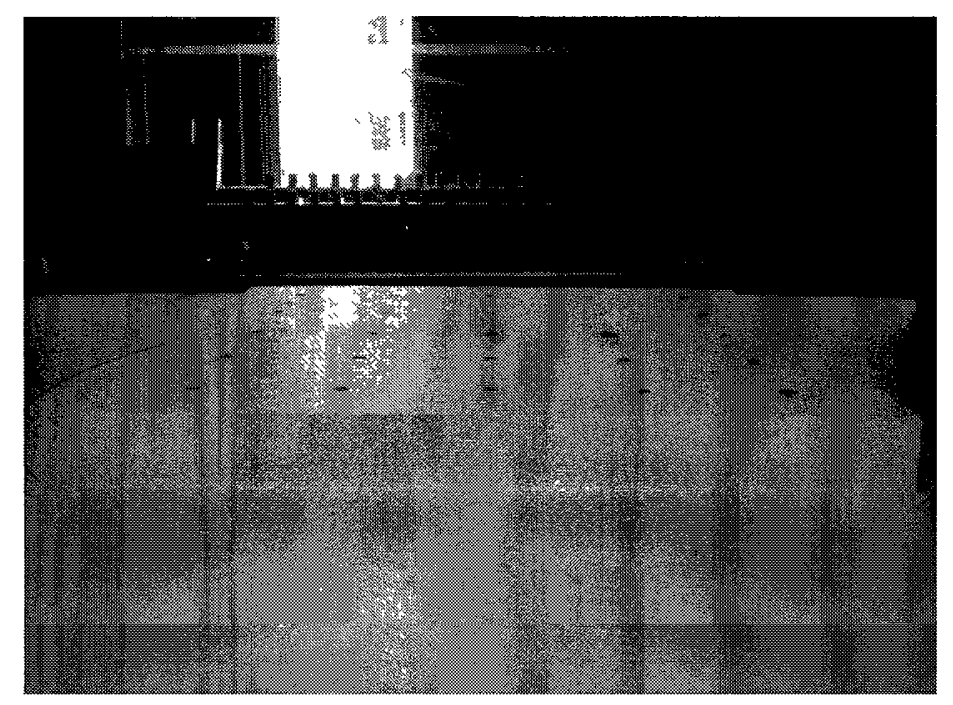

Figure 5.10: Nearby dummy panel at position $N$

The dummy panel is shown at position N. In certain cases there may be more than two panels on a roof top. There may even be an entire roof top area filled with panels. Such a field of panels will likely change the wind flow over each panel. The effect could vary for different sizes of panel fields. The first field size tested is a small field, shown in Figure 5.11.

Figure 5.11: Panel submersion in small panel field

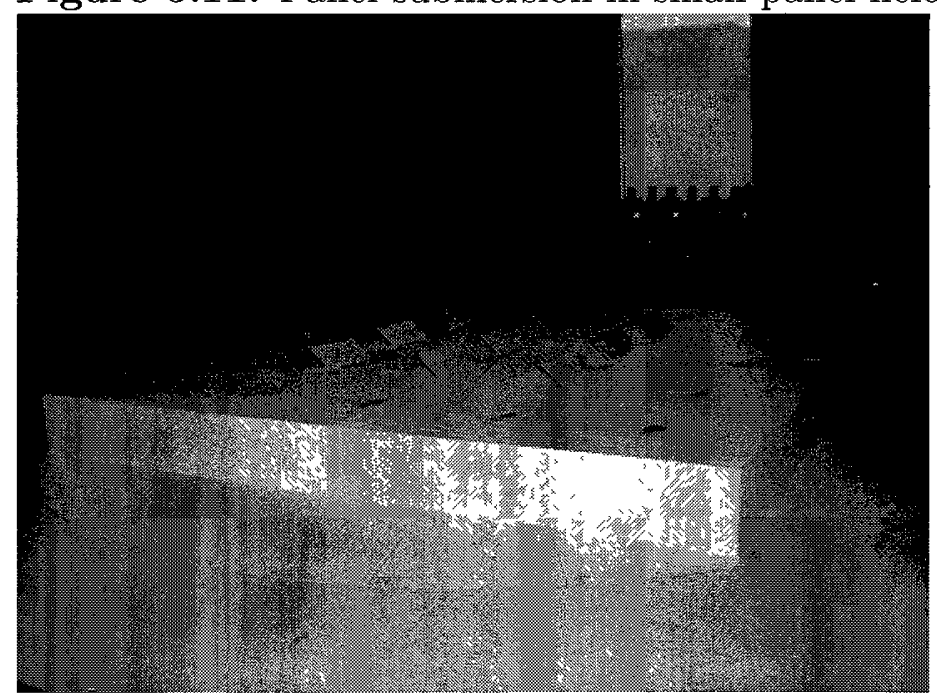


This tight cluster of panels was then expanded to a large cluster that covered the entire roof top, as shown in Figure 5.12.

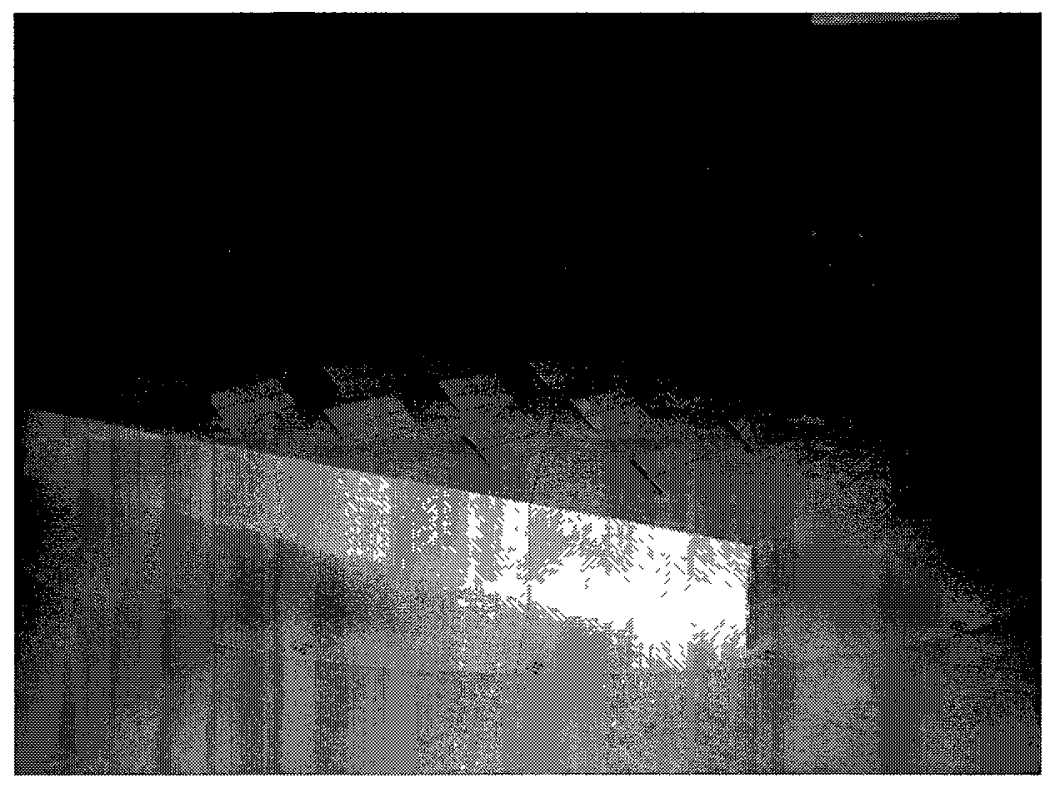

Figure 5.12: Panel submersion in large panel field

Finally, the size of the cluster field is not changed, but instead the density of the field is changed. In Figure 5.13, the full roof top field density is halved. 


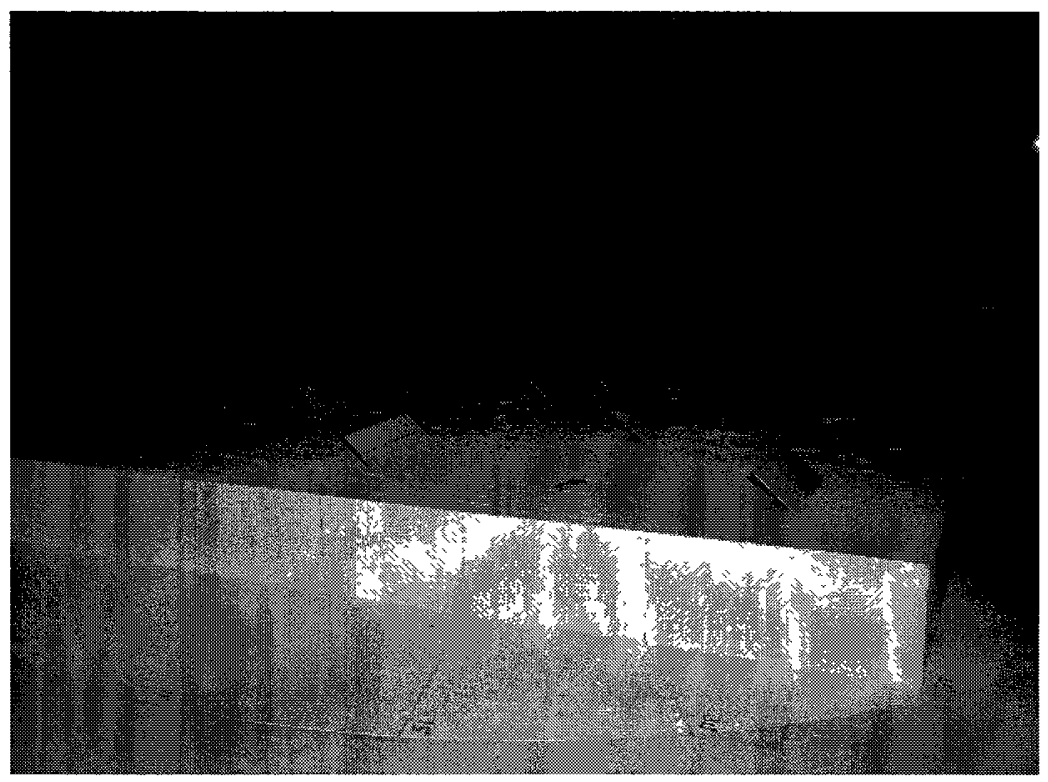

Figure 5.13: Panel submersion in sparse panel field

This concludes the different configurations in which the experiment will be performed. Next, an overview of the data acquisition unit will be presented.

\subsection{Data Acquisition}

The process of collecting data required two key pieces of equipment. The first being a load cell that could take measurements in all three directions, and the second being a software package that could collect data from this piece of hardware. This section will describe the capabilities and limitations of each unit, and the method it uses for collecting and presenting data.

\subsubsection{Multi-axis force platform}

The HE6X6 system is a commercial product that is a compact tool that can measure very low loads. It is an accurate platform that can measure the three forces and moments on one solar panel model at a time. It is designed around one piece 
Hall-Effect sensor elements with an extremely high overload protection of up to one hundred times the given capacity. Sensor electronics are built in so as to provide a digital output to a computer. Mounting holes are present to secure the solar panel model and to secure to the platform to a surface, located on the top and bottom, respectively. The platform can be seen below in Figure 5.14.

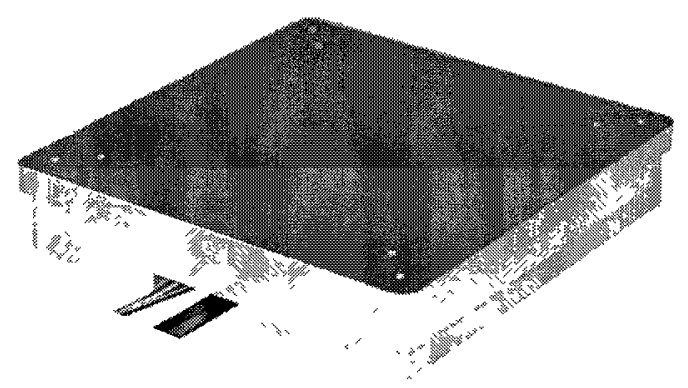

Figure 5.14: HE6X6 low load miniature force platform [18]

The platform is oriented with positive $\mathrm{X}$ pointing downstream and positive $\mathrm{Z}$ pointing into the ground, as shown below in Figure 5.15.



Figure 5.15: Orientation of force plate to wind tunnel flow 


\section{Hall-Effect Sensor}

The Hall-Effect is a method that can be used to measure displacement of a conducting coil moved through a magnetic field. By measuring the voltage generated, the force and moment can be found. The Hall-Effect force plate used measures reactions using three Hall-Effect sensors in the X, Y, and Z direction placed at each of the four corners. A letter designation is given to each corner and given a specific local sign convention, as shown in Figure 5.16. Note the local and global sign conventions, with $\mathrm{Z}$ always being positive down.

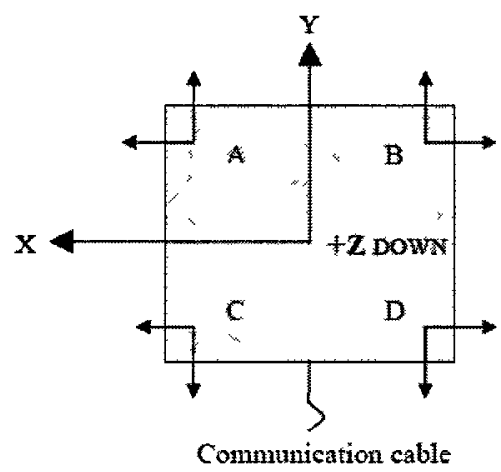

Figure 5.16: Hall-Effect sensor locations [18]

\section{Setup}

The digital signal is transmitted using a USB cable. This connector runs from the interface box to the user's computer connection. An RJ cable sends commands to the platform and receives transmissions from the platform via the interface box. Refer to Figure 5.17 [18] below for a diagram of the setup. 


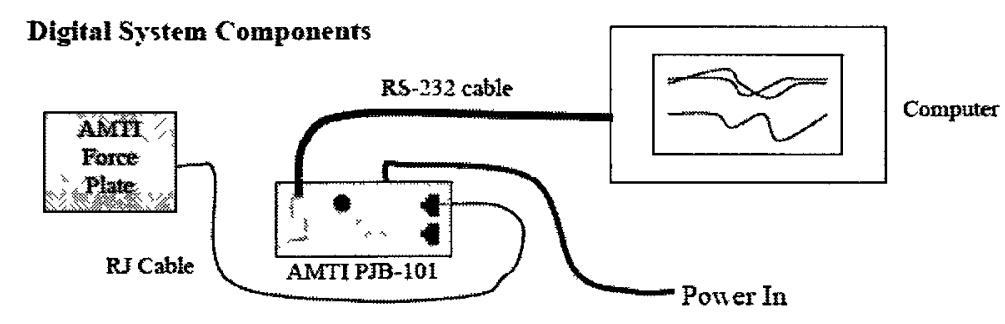

Figure 5.17: Force plate hardware layout [18]

\subsubsection{Acquisition Software}

NetForce from AMTI is a data acquisition program that is designed for use with AMTI's multi-axis force platforms for industrial force measurements. [19]

Real-tıme Data Acquisition is a key component of the NetForce software. Its realtime display mode allows the simultaneous display of up to 12 channels of data and real-time graphs. [19]

Main Screen Functions: The software presents a main screen that displays graphics of data acquisition and real time controls allowing control of the acquisition process. Controls and features include a real time graph display, a main menu, the trigger indicator, test information, and test sequence button group. These functions are briefly described below:

\section{Real Time Graph Display}

Two graphs display the force and moment signals in real time as the data is acquired. Channel Enable Buttons are used to enable or disable the six individual channels representing the three forces and three moment axes. The Channel Position Indicator is used to show the zero position for the associated channel. The setup button allows the user to set the vertical scale and color of the displayed data channel. 


\section{Trigger Indicator}

This will indicate when a trigger event has been detected. When the event is detected, the small window on the left side of the screen flashes, and the progress towards completion of the sample duration is shown on the long bar to the right side of the screen.

\section{Test Sequence Button Group}

This group of buttons provides the operating controls for acquiring and storing data. Before the test sequence begins, it is important for the user to go into the start-up section and perform a system check, calibration, and hardware zero. [19]

1. Press Start to begin real time data acquisition and initialize the protocol

2. With external forces removed, press the Tare button

3. Press the Arm, which readies the trigger logic

4. Trigger logic will sense the trigger event and stop the acquisition accordingly

5. View the data on the screen, and if it is acceptable press the Save button

6. Press Next to proceed to the next step in the protocol.

\section{NetForce Operation}

The start button is used to start and stop the data acquisition process. The data rate and duration can be set in the acquisition settings area. When the duration runs out the data will be written again in a circular buffer over the old data. Data is continuously gathered until either the stop button is pressed or a trigger event occurs.

Results can be saved into either text files or binary BSF files. In the text files each column of data represents a channel of the installed force platform. Each row consists of data taken at a given moment in time. Text files are comma delimited with data 
columns representing each channel $F_{x}, M_{x}$, etc. Tare values are subtracted from the data. The option exists for text files to be exported to a spreadsheet program as well. The BSF files can keep track of and save trial descriptions, the date, etc. [19]

Under the settings menu, a hardware zero option exists to perform an internal auto zero which will remove most of the offset caused by thermal drift.

\section{Trigger Method}

Various types of events may be used as the method by which the system is triggered to collect data. The option for no trigger also exists, which causes the system to immediately start collecting data once the test sequence is started. Data collection can also begin when the system experiences a certain channel level of data, or experiences a fall or rise in data magnitude. An elapsed time can also be set to pass before the data begins recording. A keystroke may also be implemented to signal the start of data acquisition. Sample duration can be set to determine the amount of time the system will take to acquire data. The Acquisition Rate allows control over the amount of recordings per second, from $50 \mathrm{up}$ to $1200 \mathrm{~Hz}$. [19] This thesis will use $50 \mathrm{~Hz}$ over 100 seconds duration.

\section{Platform Range}

Under the review settings dialog box, information such as resolution, and sensitivity can be viewed in either English or metric units. Such values are displayed for each of the six channels: $F_{x}, F_{y}, F_{z}, M_{x}, M_{y}$, and $M_{z}$. The electrical range reports the $\max$ and min force or moment that can be read by the platform and software. The mechanical range reports the max and min force or moment that the platform can physically endure. The resolution is given in terms of force or moment per bit, and reports the maximum sensitivity that the platform can detect. [19] 


\subsubsection{Platform Calibration}

The force platform is shipped from the factory after being calibrated on site. However, this calibration should still be checked so that results can be trusted. The six modes that must be calibrated are the $\mathrm{x}, \mathrm{y}$, and $\mathrm{z}$ direction force and moment measurements.

\section{Force Calibration}

For the force in the $\mathrm{z}$ direction, the force plate was placed on a level surface and defined masses were placed on the platform as shown in Figure 5.18 and weighed using the NetForce acquisition software.

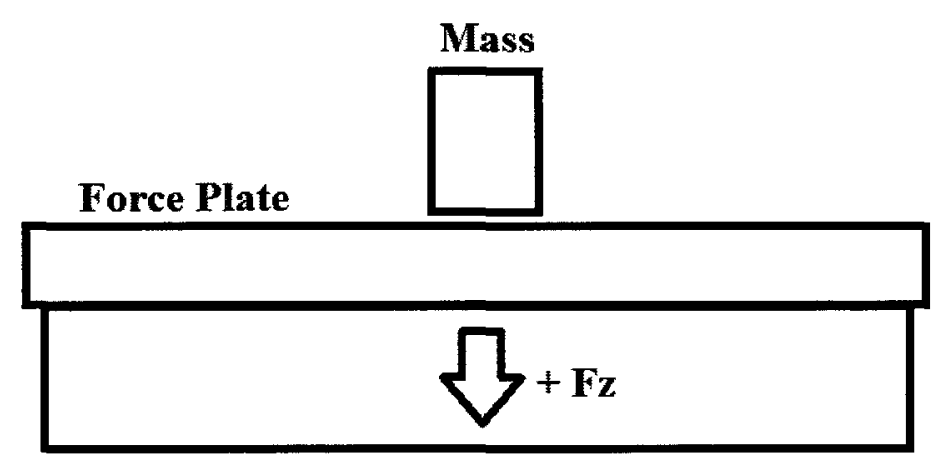

Figure 5.18: Platform setup for $F_{z}$ calibration

The resultant forces are presented in Figure 5.19 below: 


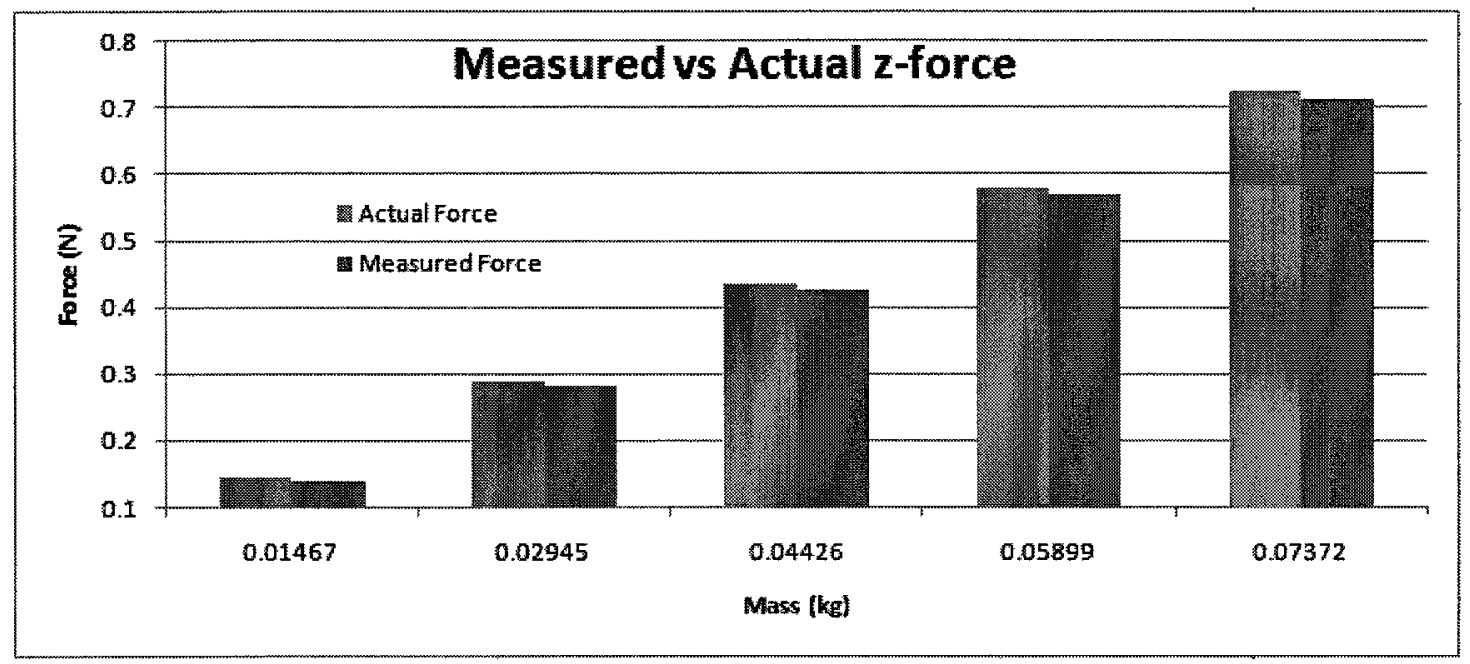

Figure 5.19: Measured vs Actual Z-force

For the force in the $\mathrm{x}$ direction, defined masses were placed on the platform's edge while it was turned sideways and secured in a level position on the x-plane as shown in Figure 5.20 and weighed using the NetForce acquisition software. 


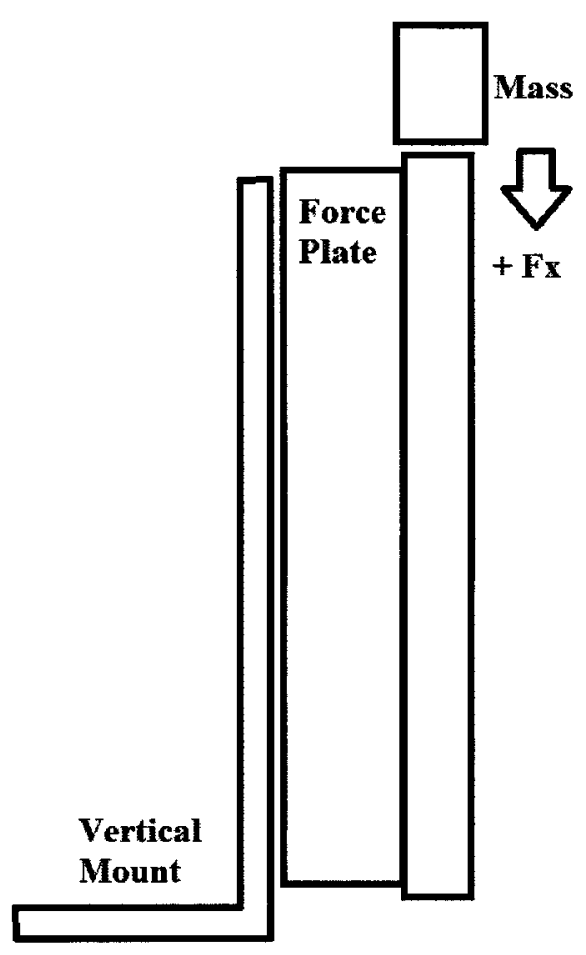

Figure 5.20: Platform setup for $F_{x}$ calibration

The resultant forces are presented in Figure 5.21 below:

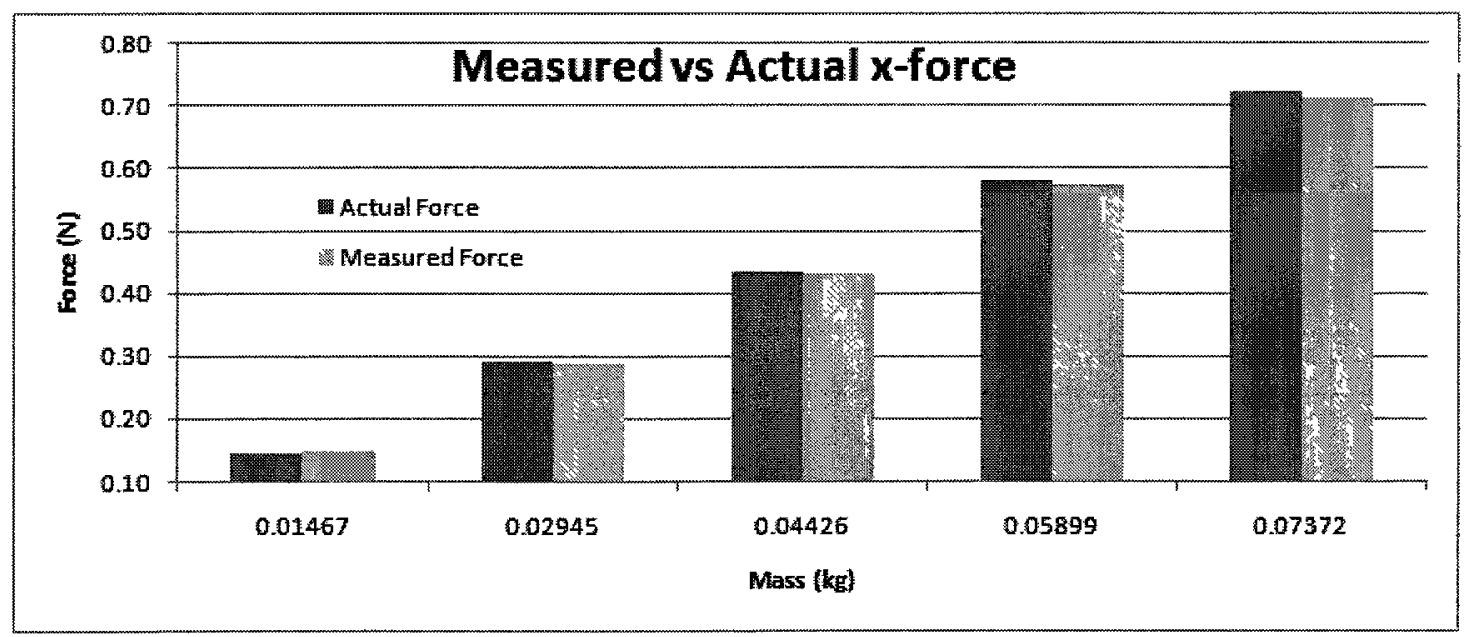

Figure 5.21: Measured vs Actual X-force

For the force in the y direction, defined masses were placed on the platform's edge 
while it was turned sideways and secured in a level position on the y-plane as shown in Figure 5.22 and weighed using the NetForce acquisition software.

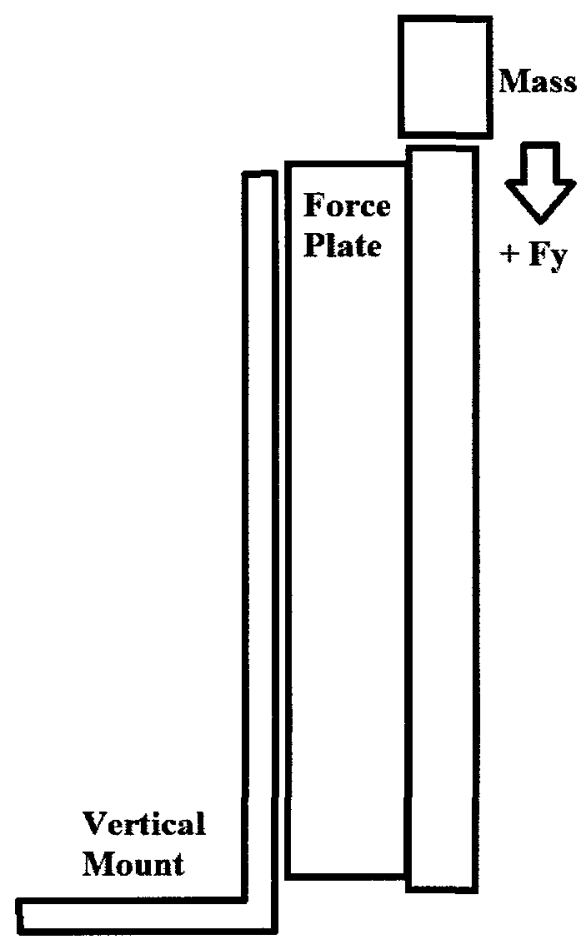

Figure 5.22: Platform setup for $F_{y}$ calibration

The resultant forces are presented in Figure ?? below: 


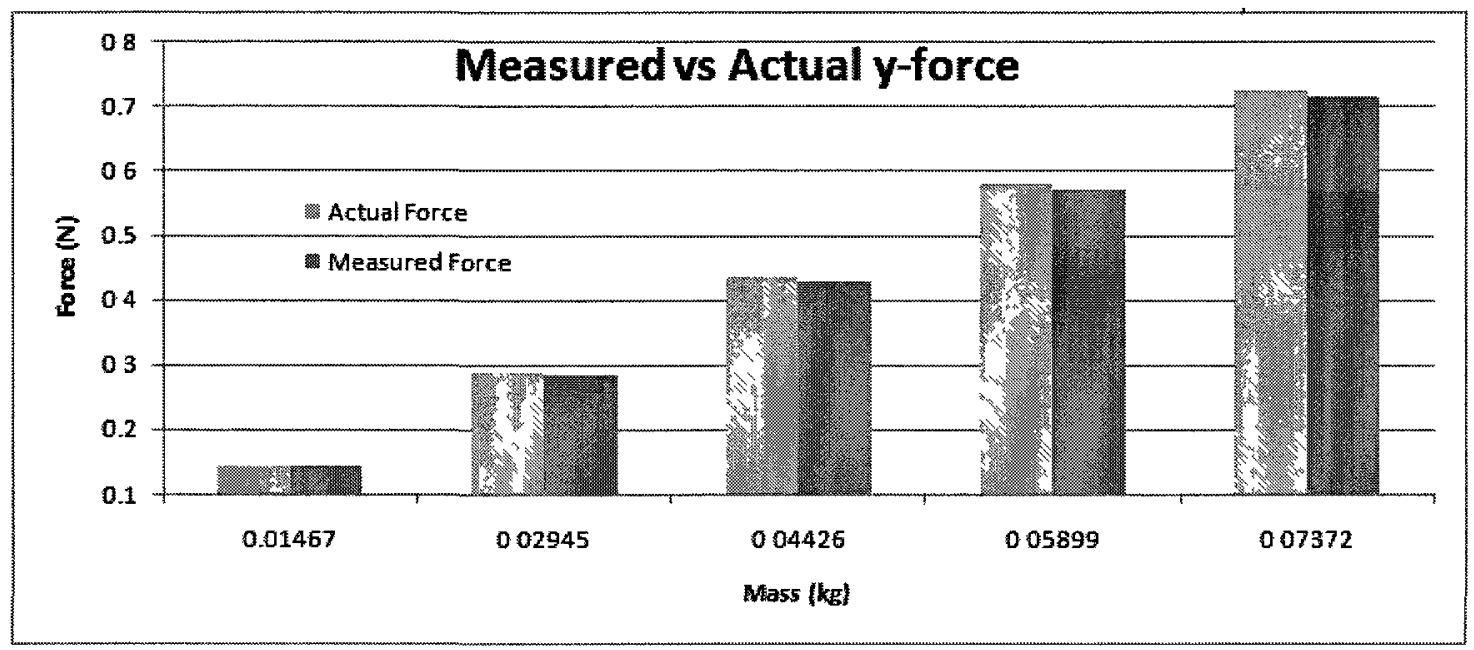

Figure 5.23: Measured vs Actual Y-force

As seen, the forces measured are offset from the actual forces being represented. To establish the existence of a possible trend, Figure 5.24 below presents a direct comparison of the forces measured to the established force values.

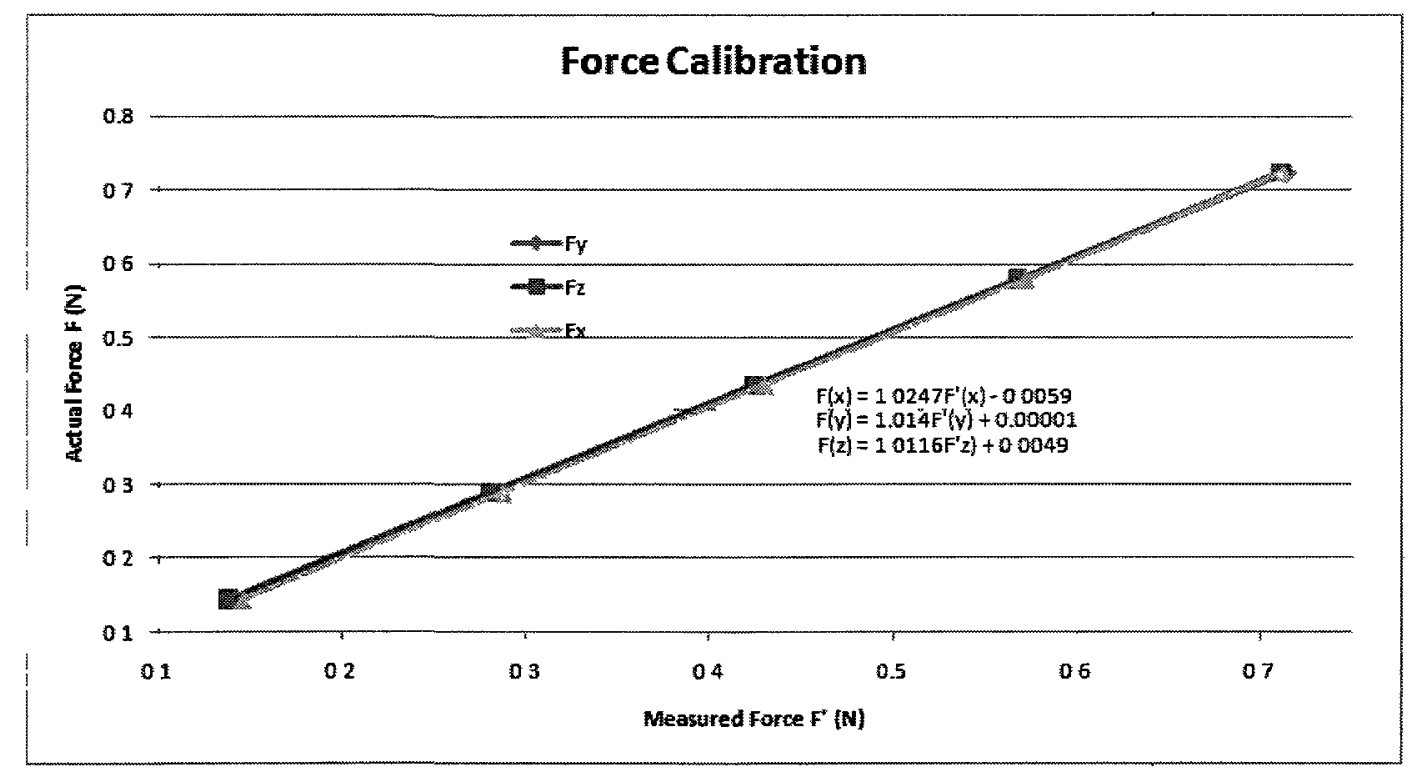

Figure 5.24: Force calibration

A trend line does indeed exist. The trends for $F_{x}, F_{y}$, and $F_{z}$ are linear, and can 
thus be used to correct the experimental forces measured to the actual forces.

\section{Moment Calibration}

For the moment on the $\mathrm{z}$ axis, with the platform secured on its side, an aluminum beam was bolted to the plate's surface. After the force plate was zeroed, a defined mass was placed at varying positions along the extended rod, as shown in Figure 5.25.

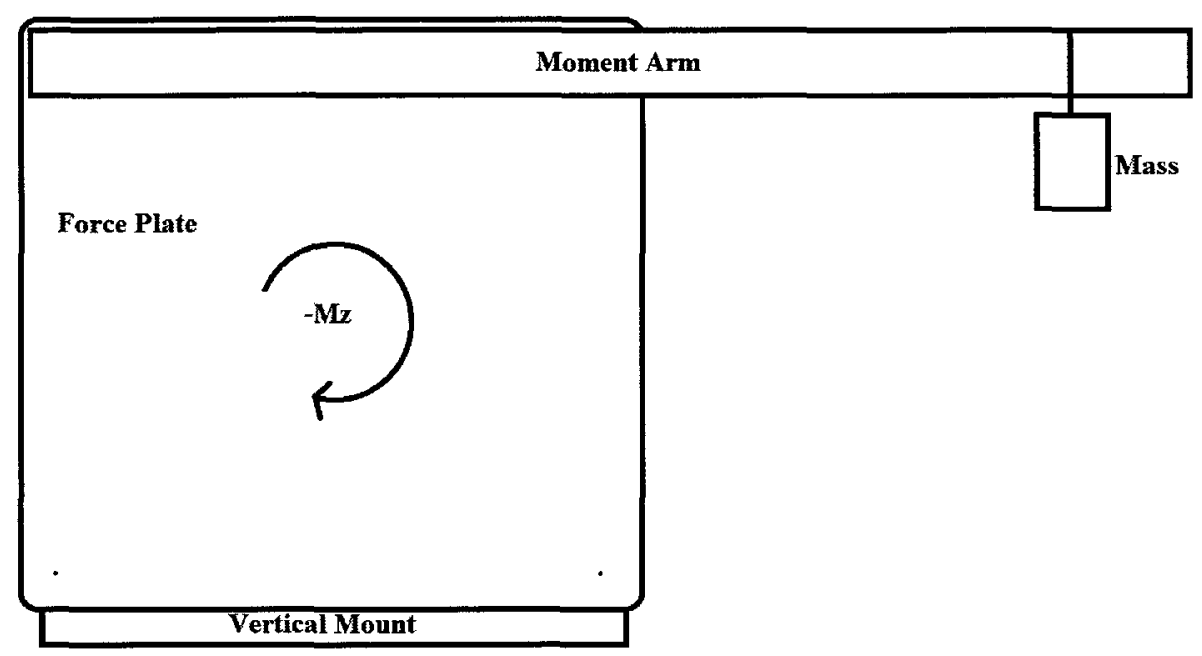

Figure 5.25: Platform setup for $M_{z}$ calibration

The resultant moments were measured using the NetForce software and exported into a notepad file. This data was then imported into a spreadsheet program, where the moments were plotted against time. This allowed for a range of data to be selected that had reached a steady state and could be averaged to retrieve the subjected moment. The results are presented in Figure 5.26 below: 




Figure 5.26: Measured vs Actual Z-moment

Surprisingly, a large difference in results are seen in the $F_{z}$ calibration. This could be due to a manufacturing problem, or an issue with inaccurate calibration techniques. It is suggested that an alternative method be used to check calibration in future work. For the moment on the $\mathrm{x}$ axis, the force plate was secured in the z-plane. An aluminum beam was then bolted to the plate's surface. The bending axis aligned with the $\mathrm{x}$-axis. After the force plate was zeroed, a defined mass was placed at varying positions along the extended rod, as shown in Figure 5.25.

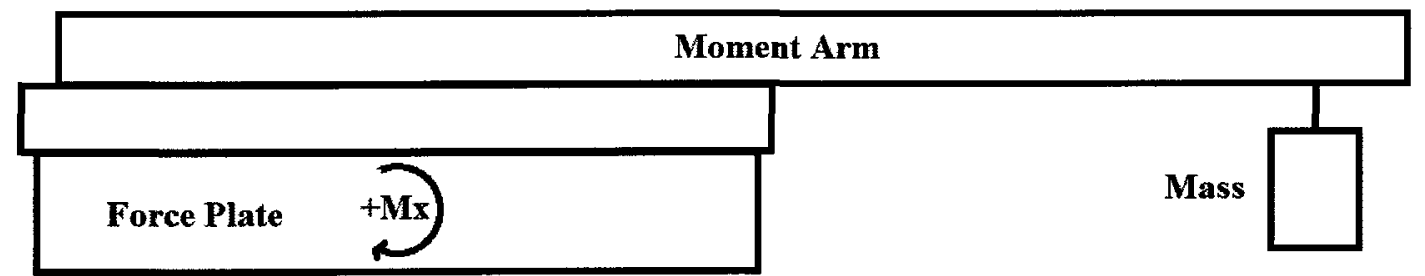

Figure 5.27: Platform setup for $M_{x}$ calibration

The resultant moments were measured using the NetForce software and exported 
into a notepad file. This data was then imported into a spreadsheet program, where the moments were plotted against time. This allowed for a range of data to be selected that had reached a steady state and could be averaged to retrieve the subjected moment. The results are presented in Figure 5.28 below:

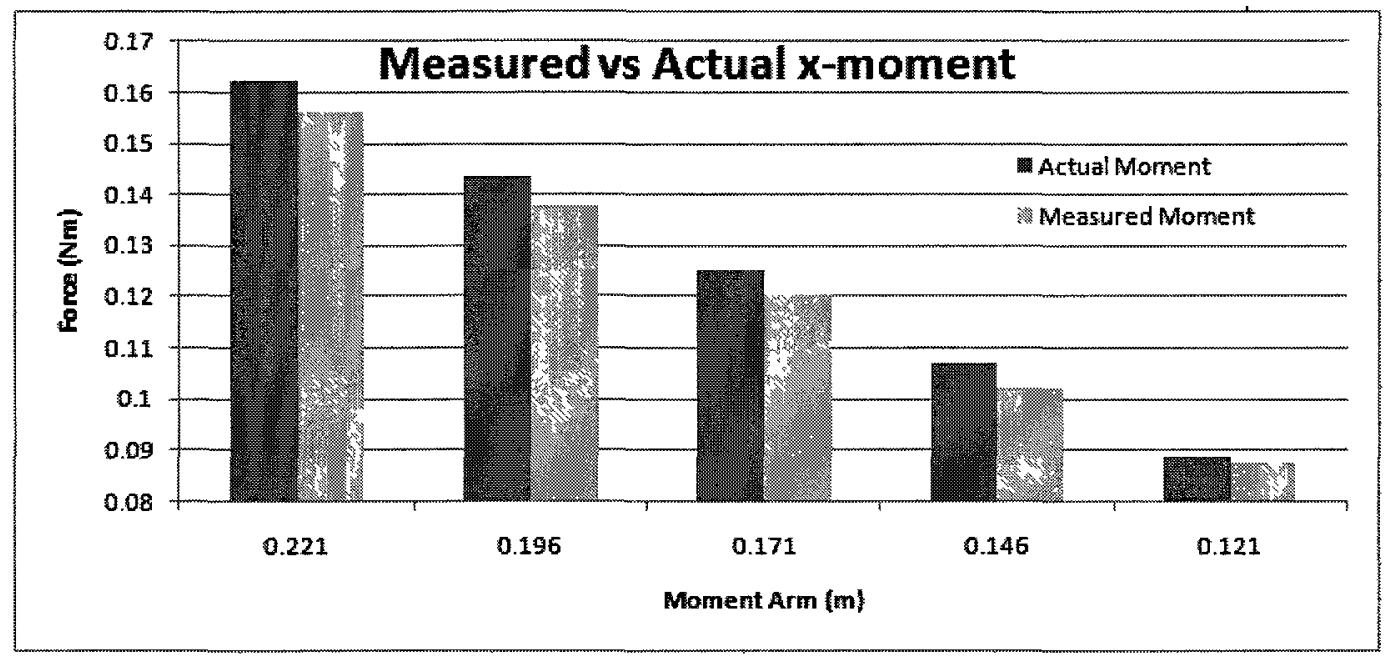

Figure 5.28: Measured vs Actual X-moment

For the moment on the $y$ axis, the force plate was secured on the z-plane. An aluminum beam was then bolted to the plate's surface with the bending axis aligned with the y-axis. After the force plate was zeroed, a defined mass was placed at varying positions along the extended rod, as shown in Figure 5.29.

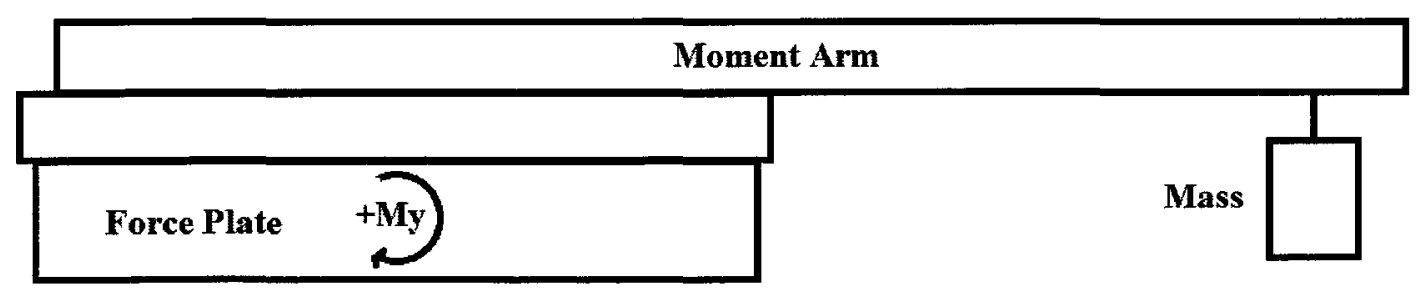

Figure 5.29: Platform setup for $M_{y}$ calibration 
The resultant moments were measured using the NetForce software and exported into a notepad file. This data was then imported into a spreadsheet program, where the moments were plotted against time. This allowed for a range of data to be selected that had reached a steady state and could be averaged to retrieve the subjected moment. The results are presented in Figure 5.30 below:

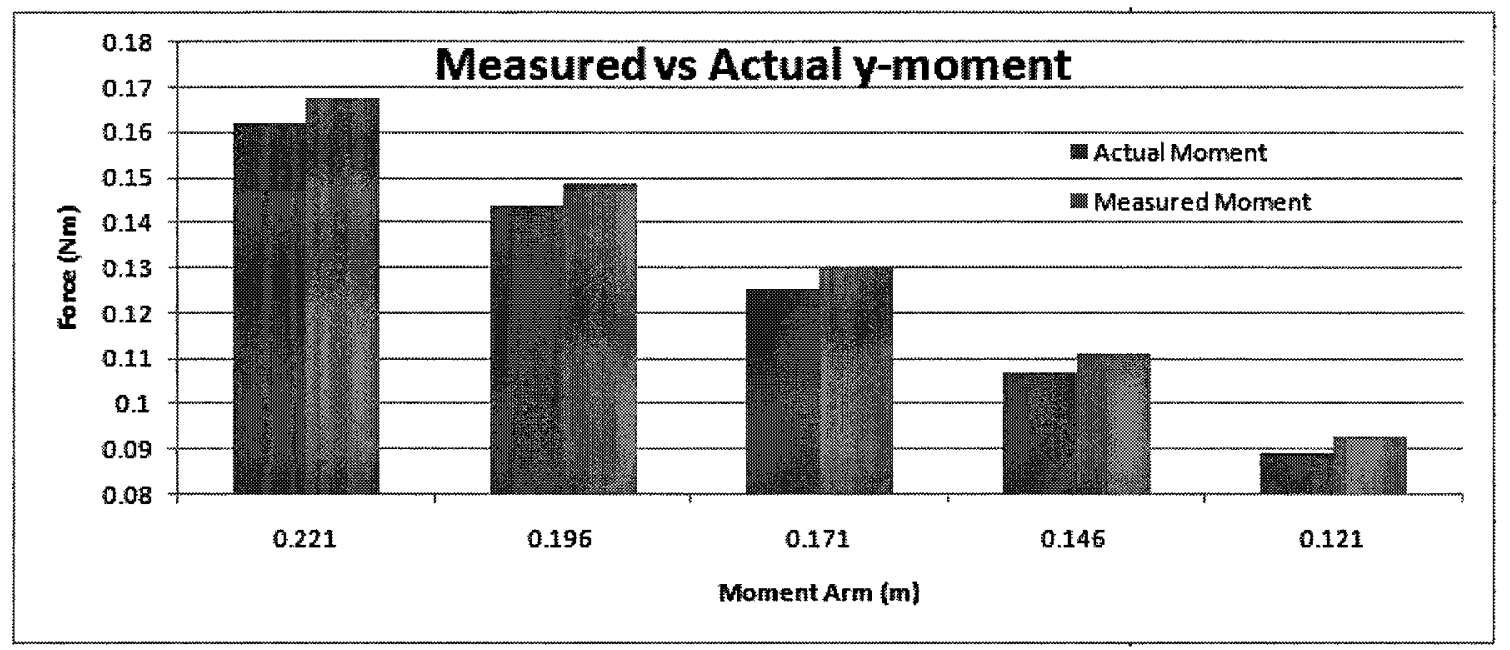

Figure 5.30: Measured vs Actual Y-moment

As seen, the moments measured are offset from the actual forces being represented. To establish the existence of a possible trend, Figure 5.31 below presents a direct comparison of the moments measured to the established moment values. 


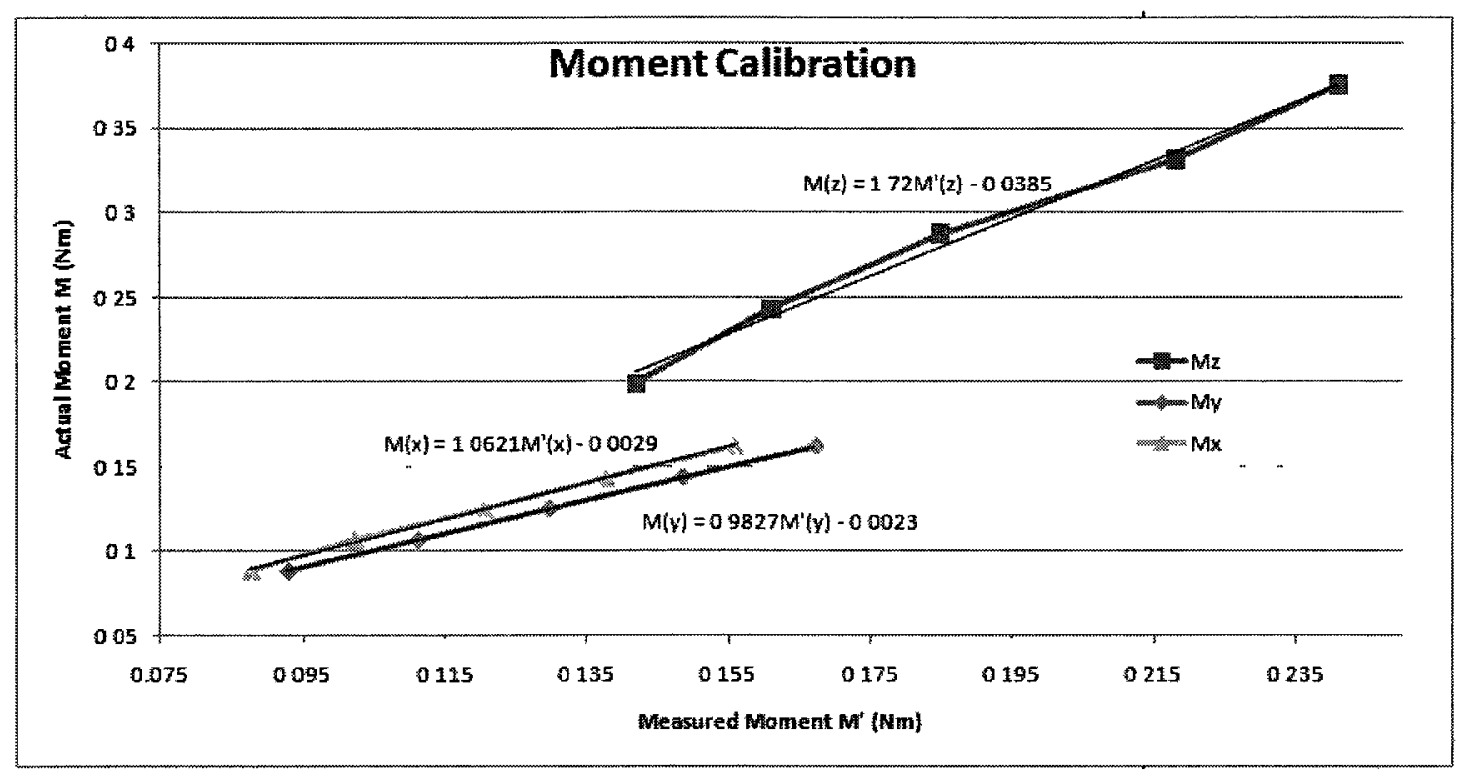

Figure 5.31: Moment calibration

A trend line does indeed exist. The trends for $M_{x}, M_{y}$, and $M_{z}$ are linear, and can thus be used to correct the experimental moments measured to the actual moments.

\subsection{Uncertainty Analysis}

Since the resulting forces and moments measured from the force plate are determined from other variables, it must be considered how the uncertainties in each channel's data propagate into the resulting force or moment. This section will present the results of the Taylor Series Method [20] applied to define how certain the force plate results are. The final equation format, derived in Appendix A, is shown below.

$$
\left(\frac{U_{F_{x}}}{F_{x}}\right)_{\text {relattveuncertainty }}=\frac{U_{A x}}{F_{x}} \sqrt{\left(S 11^{2}+S 12^{2}+\ldots\right)}
$$

In this equation, $S$ is a value from the calibration coefficient matrix included with 
the software, and it further explained in Appendix A. The bit data from the Hall Effect sensors produce up to 2048 bits of data, with a relative uncertainty $\left(U_{A_{x}}\right)$ of $0.6 \%$ [19]. This process will reduce the equation to a relation between the relative uncertainty in the experimental result,i.e. $F_{x}$, to the relative uncertainties in the measured variables, i.e. $A_{x}, B_{y}, D_{z}$, etc, and the force or moment, i.e. $F_{x}, M_{y}$, being measured. The remaining variable of $F_{x}$ in this equation will be varied to determine how its measured result is certain. In Figure 5.32, the relative uncertainty for the force measurements are presented.

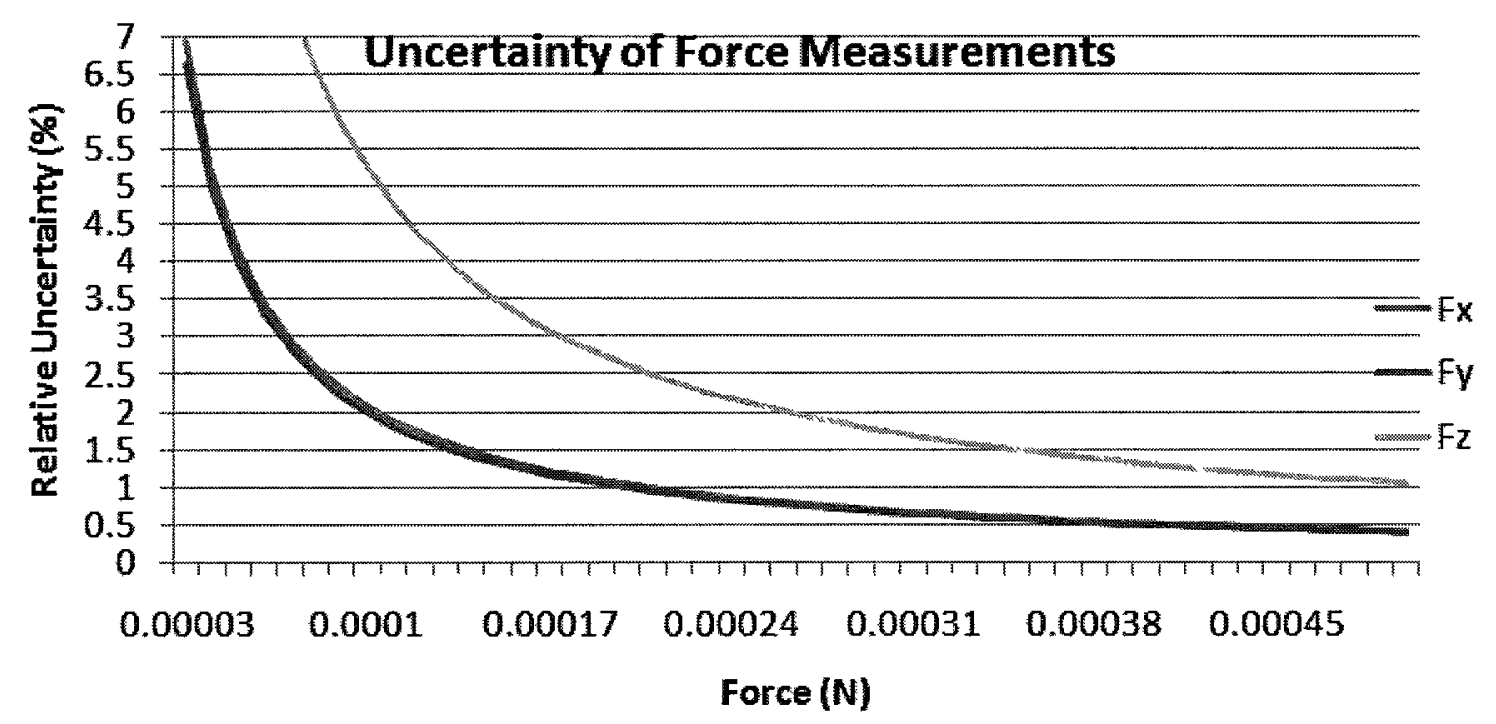

Figure 5.32: Estimated uncertainty of forces

As seen above, the uncertainty below $0.00016 \mathrm{~N}$ is fairly large, and results below this should be trusted with caution. In Figure 5.33 below, the relative uncertainty for the moment measurements is presented. 


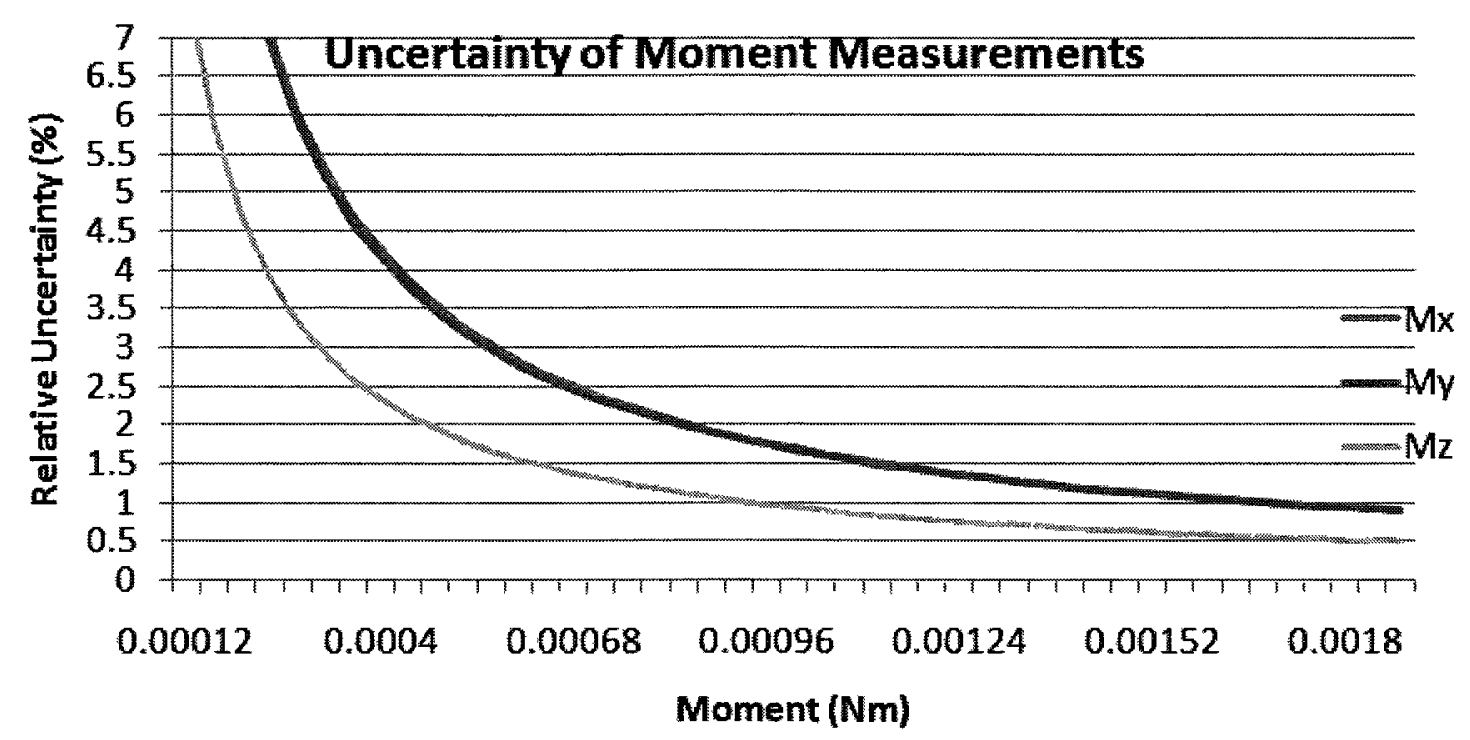

Figure 5.33: Estimated uncertainty of moments

As seen above, the uncertainty below $0.00056 \mathrm{Nm}$ is fairly large, and the results below this should be trusted with caution.

\subsection{Sources of Error}

Throughout the testing and analysis phase of the project, certain issues arose that brought awareness to aspects of the experimental methods that could reduce the accuracy and reliability of the results. These sources of error extended from two means, that the experiment was not designed to its greatest strength, and the other being that the experiment not was performed as accurately as it could have. These means are explained in the following subsections.

\subsubsection{Design Considerations}

Aspects of the wind tunnel experiment setup were discovered to have a possible effect on the results. These were mostly due to the solar panel model itself, and how it was 
connected to the force plate.

The composition of the panel is made of ABS plastic. With the small dimensions of the plastic model, ABS does not produce a high level of rigidity in the model. During the experiment, it was assumed that the model was completely rigid. However, by simply holding the model it is evident that a degree of flexibility does exist. This flexibility in the plastic model leads to two problems. Firstly, the entire model has the possibility of flexing under a wind load. The flex could most probably occur around the Z-axis, or bend backwards along the Y-axis. Such deflections can change the aerodynamics around the panel to the point where a different load is produced.

Material deflection also leads to the next possible problem, that of aeroelastic effects. The wind forces acting on the panel can create deflections that are countered by the material elastic forces, which in turn with inertial forces can create an oscillating motion. This motion could be found in multiple modes, such as along the $Y$-axis with the panel leading and trailing edges, or the $\mathrm{X}$-axis with the panel edge tips. These oscillations would not only affect the measured forces, but also affect the frequency at which vortex shedding is detected.

Finally, the method used to connect the panel strut to the force plate could cause offset results. The method of attachment, which is that of interference fit, does not necessarily lock the strut firmly into the force plate. Although a rigid connection was assumed, bending or twisting of the panel with excessive force can result in the strut slightly shifting its attachment position along the Z-direction or any of the axes.

\subsubsection{Human Error}

Operating the experiment proved to be challenging at times due to the precision involved in setting up each wind tunnel test case. Angles and distances were required to be measured, which were subject to the possible mistakes from misalignments.

Calibrating the load cell for moments required placing a mass at various distances 
along a moment arm. These placements were done by aligning the mass with markings painted onto the moment arm. Due to the sensitivity of the load cell, being off even by a few milimeters could changes the calibration results. This could account for the large moment calibrations required, especially for the Z-axis.

The building model was marked with the angles required for testing. Marking the angles, although done with as much precision as possible, was done with a protractor only about one tenth the diameter of the turn table. Extending the lines to the edge of the turn table could prove to be inaccurate by a few degrees. Thus when rotating the building to its desired yaw angles may be simulating wind directions a few degrees from that desired.

The solar panel model itself also follows a set of angles when its azimuth angle is changed. These are marked very accurately since they are within the size of the protractor. However, aligning the panel with these angles presents a challenge. Since markings are on the rooftop surface, and the panel is a centimeter or so above the rooftop, a degree of visual skill is needed to align the panel with the markings. This could result in azimuth angles being offset a few degrees.

Adjustment of the solar panel model proved difficult not only when aligning angles but also in the height of the solar panel. No system was established for determining the height of the solar panel off the rooftop surface. This distance was judged based on the length of stem remaining at the bottom of the strut. This vertical distance could have varied by a few millimeters, and thus closed or widened the gap between the leading edge of the panel and the rooftop surface. Changing this gap size could have made an effect on the flow blockage of the panel model, and thus the force exerted on it by the passing wind flow.

Similar issues were also experienced with not just the active panel being measured, but the dummy panels surrounding the active panel during tests that involved surrounding panel field sizes and nearby panel effects. Although forces on the dummy 
panels were not measured, the wind flow around them affects the aerodynamic properties of the flow around the active panel. Positioning up to 24 dummy panels at both the correct height off the rooftop as well as the correct azimuth angle would be very difficult to perfect by hand. Thus, it is likely that some of the dummy panels were not positioned at the correct height or angle, but instead off $M_{y}$ a few degrees or millimeters. Fortunately, as seen in the results section, only a small case of nearby panels affected the active panel's flow, and it is likely that minor changes in dummy panel positioning would not produce a change in results.

Finally, the ultimate alignment issue partakes to the force plate load cell itself. Situated underneath the wind tunnel floor on a tripod, it can be difficult to adjust the orientation of the force plate so that it is at a right angle to the floor, and aligned with the wind flow direction. Leveling the force plate with the ground is done with a small hand level, while alignment with the wind flow is done with the right angle triangle visually guiding the side of the force plate to be in line with the tunnel floor structural beam. Since these techniques are visually based, it is likely that they could be misaligned by a degree or two. Although this and the previous mentioned sources of error could contribute to inaccuracy in specific numerical values, relatively the results are still reliable in terms of trends observed, since the data found physically makes sense. 


\section{Chapter 6}

\section{Wind Tunnel Results and Discussions}

This chapter will outline and discuss the results obtained from experiments performed in the wind tunnel. The effect of the Reynolds number will be examined followed with a discussion of the data trends observed with the various wind tunnel model configurations. In the wind tunnel, solar panels of different angles of attack were monitored at different wind speeds as well as at various wind directions. Orientations and locations on top of a large retail store style building were also monitored. The effect of nearby panels was monitored as well, both by checking proximity to one panel, and proximity to a cluster of panels. The forces in each direction and moments on each axis acting on solar panel models were measured as described in the experimental set up. Each data point consisted of a 100 second sampling trial at a rate of $50 \mathrm{~Hz}$. Results for all the data collected with standard deviations may be found in Appendix $\mathrm{B}$, with non-dimensionalized results derived and presented in Appendix C. The nondimensionalized results are of significant value for potentially providing a basis to provide justification for the building code using any similar style solar panel array. 


\subsection{Theoretical Vortex Frequency}

One of the interesting results presented here and further in Chapter 7 will be a frequency analysis identi $F_{y}$ ing instances of vortex shedding. Two instances where vortex shedding can be expected are off the solar panel model, and off the leading edge of the building. These two instances are calculated below using the Strouhal number as a dimensionless number for vortex shedding frequency.

$$
f=\frac{V S_{t}}{L}
$$

where $\mathrm{f}$ is the frequency of vortex shedding that can be expected, $\mathrm{V}$ is the wind velocity ahead of the panel model or building leading edge, $S_{t}$ is the Strouhal number, which for square cylinders is 0.18 and flat plates is 0.15 , and $\mathrm{L}$ is the characteristic length which for the solar panel model is the panel's projected chord length, and for the building's characteristic length is the building's height [10]. The freestream velocity is not used for this calculation, but instead the velocity is taken from point measurements of CFD simulations performed later in Chapter 7.

The theoretical vortex shedding frequency predicted by this process is $10.3 \mathrm{~Hz}$ for the solar panel model, and $14.1 \mathrm{~Hz}$ for the building's leading edge.

\subsection{Effect of Varying Wind Velocity}

A panel with a pitch of 45 degrees was situated in the center of the rooftop, with the panel and rooftop positioned at the zero degree mark. An example of this setup can be seen in the schematic shown in Figure 6.1. 


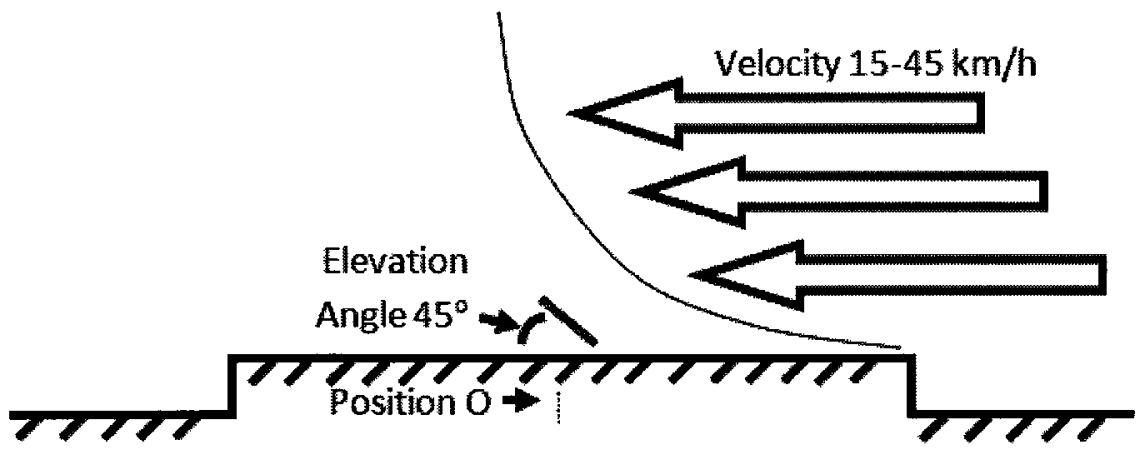

Figure 6.1: Example of schematic for velocity test case

The wind velocity was varied from 45 kilometers per hour down to 15 kilometers per hour.

In Figure 6.2 below, a composite plot of $F_{x}, F_{y}$, and $F_{z}$ is shown displaying the mean average of each time series sample as data points for each wind velocity.

\section{Effect of Wind Velocity on Reaction Force}

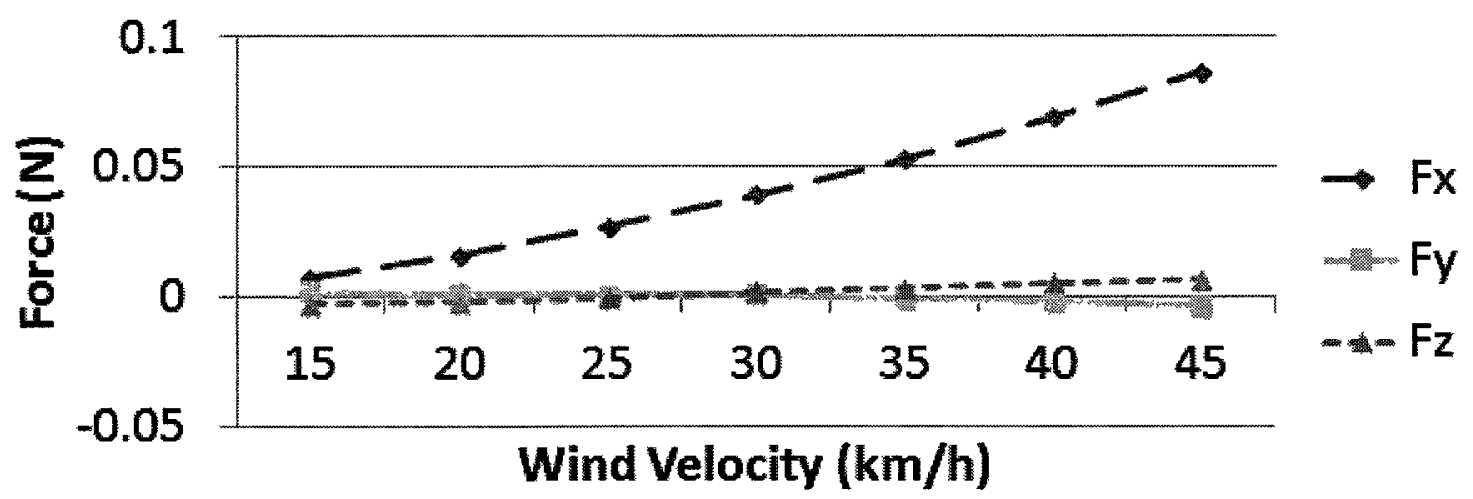

Figure 6.2: Wind velocity reaction forces

In Figure 6.3 below, a composite plot of $M_{x}, M_{y}$, and $M_{z}$ is shown displaying the mean average of each time series sample as data points for each wind velocity. 
Effect of Wind Velocity on Reaction Moments

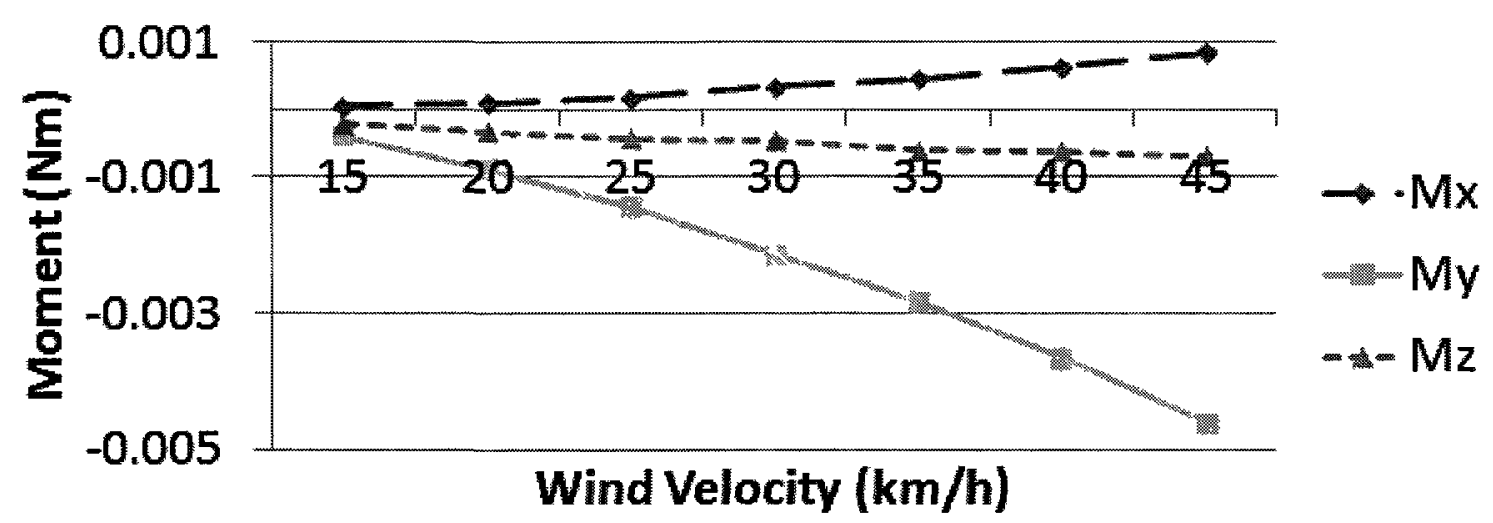

Figure 6.3: Wind velocity reaction moments

\subsubsection{Significance of Wind Velocity}

The most obvious concern for rooftop structures is strong wind gusts. Although the wind tunnel cannot operate much higher than $45 \mathrm{~km} / \mathrm{h}$, the drag coefficient can be used for full scale, full velocity models. The trend seen in forces and moments can also give an idea what the full scale model would experience. As seen in Figure 6.2, it is seen that $F_{y}, F_{z}$, and thus $M_{x}$ and $M_{z}$, show only small variation. However the most obvious trend is the traumatic increase of drag in the X-direction. This significant increase corresponds to the large increase in bending moment in the Y-axis, as seen in Figure 6.3. It can be said that the greatest concern when designing for high speed winds is the stress observed due to high forces and torques in the $F_{x}$ and $M_{y}$ direction.

\subsubsection{Reynolds Number Insensitivity}

As presented in Chapter 3, research [14] has shown that for Reynolds numbers between the range of $10^{3}$ and $3 \times 10^{6}$, the force coefficient for rectangular plates and 
sharp edged blunt objects does not vary significantly with changes in Reynolds number. The operating conditions in the wind tunnel at the panel model in the test section experience a Reynolds number of approximately 17,700 . This is based off the assumption that the velocity at the center of the panel is $11.11 \mathrm{~m} / \mathrm{s}$, the panel chord length is $0.025 \mathrm{~m}$, and the air viscosity is $1.98 \times 10^{-} 5 \mathrm{~kg} /(\mathrm{ms})$.

The Reynolds number was calculated using the equation below.

$$
R e=\frac{V L}{\nu}
$$

Since the Reynolds number is within the range limit described in literature research [14], this theory can be applied to the panel testing in the wind tunnel. This means that as long as the wind tunnel velocity keeps the Reynolds number within the prescribed range, the boundary layer separation point remains unchanged and the turbulence forces at the small scale may be proportionally scaled to full scale using the force coefficient.

However, the wind tunnel test section setup is not just a simple plate in a free stream flow. The panel itself does not have a completely smooth and uniform front and rear surface, and is located within the thick boundary layer of the wind tunnel, as well as the boundary layer of the building. This warrants a confirmation check to ensure that with a constant velocity change, the forces experienced change inversely proportional to the square of velocity. This data was presented in Figure 6.2. As seen, the forces change in a shallow parabolic fashion as the velocity increases. To relate this to the drag coefficient the following equation is examined:

$$
C_{d}=\frac{2 F_{d}}{\rho V^{2} A}
$$


Assuming that $\rho$ and $\mathrm{A}$ are constant, to have a constant $C_{d}$ the force must be proportional to the velocity as follows

$$
F_{d} \alpha V^{2}
$$

This trend is observed in how the velocity affects both the force, and the moments in Figure 6.3. It can therefore be said that $C_{F_{x}}, C_{F_{y}}, C_{F_{z}}, C_{M_{x}}, C_{M_{y}}$, and $C_{M_{z}}$ are insensitive to changes in velocities, and assuming the viscosity remains constant, is also insensitive to changes in Reynolds number within the $10^{3}$ and $3 \times 10^{6}$ range.

\subsection{Effect of Varying Panel Elevation Angle}

A panel submersed in wind flow at 40 kilometers per hour was situated in the center of the rooftop, with the panel and rooftop positioned at the zero degree mark. The panel angle was varied from 60 degrees down to 15 degrees.

In Figure 6.4 below, a composite plot of $F_{x}, F_{y}$, and $F_{z}$ is shown displaying the mean average of each time series sample as data points for each panel angle.

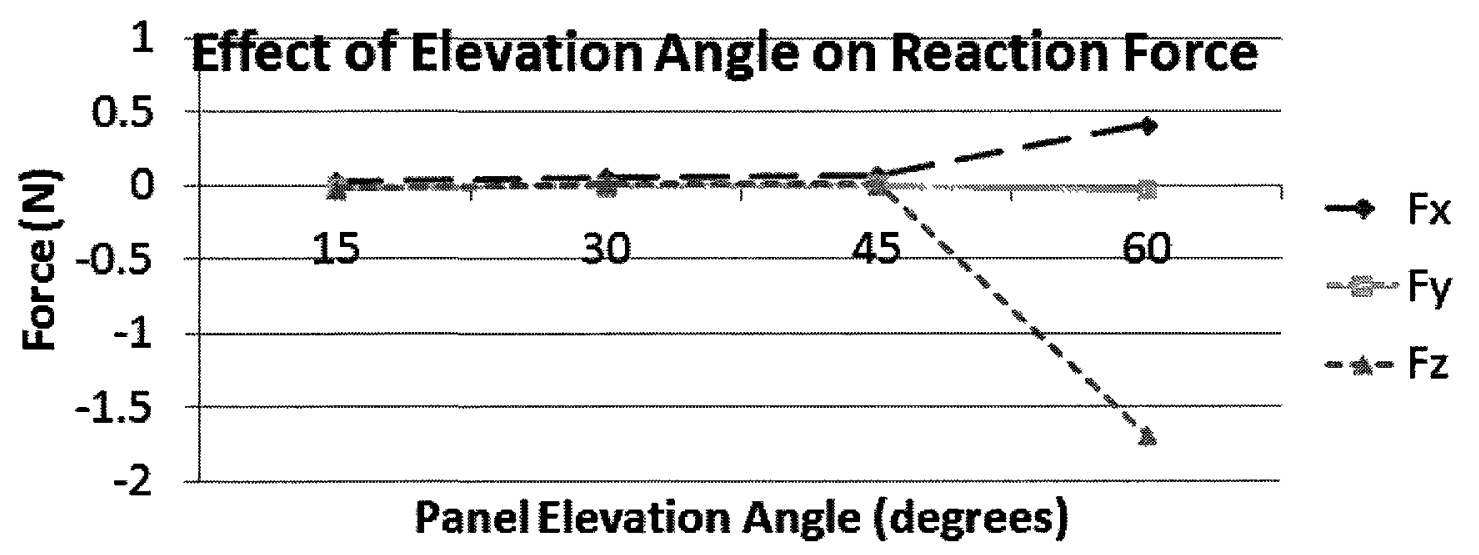

Figure 6.4: Panel elevation angle reaction forces

In Figure 6.5 below, a composite plot of $M_{x}, M_{y}$, and $M_{z}$ is shown displaying the 
mean average of each time series sample as data points for each panel angle.

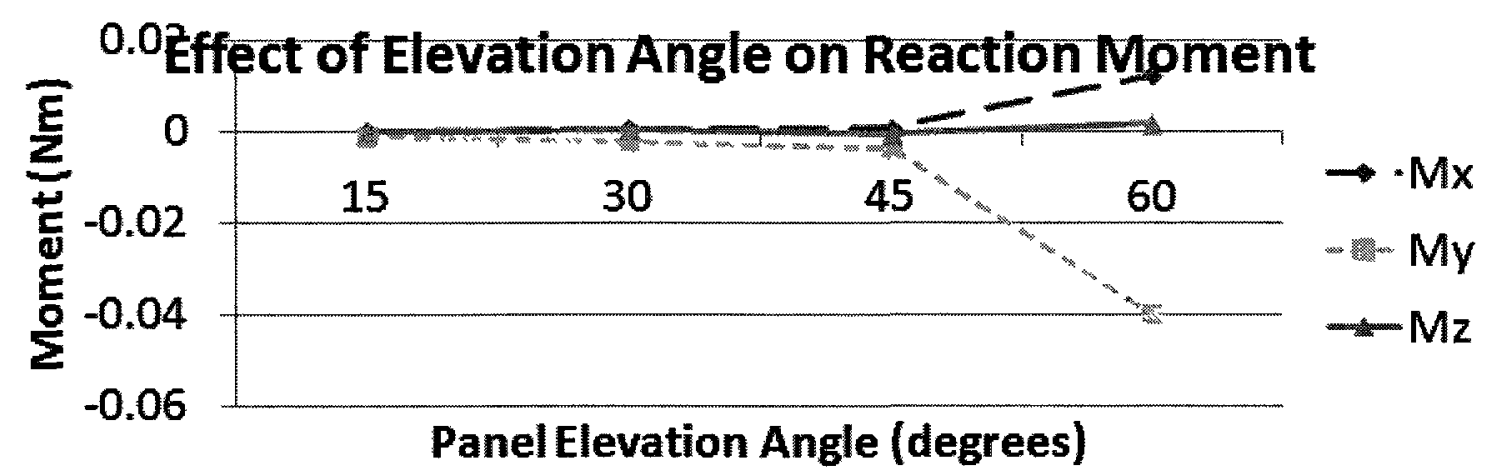

Figure 6.5: Panel elevation angle reaction moments

\subsubsection{Significance of Panel Elevation Angle}

An interesting trend was noticed in the force and moment measurements taken while the panel elevation angle was varied. As seen in Figures 6.4 and 6.5, the variation of $F_{y}$ and $M_{z}$ is practically unchanged. However, it is seen that there is a significant increase in drag $\left(F_{x}\right)$ and lift $\left(F_{z}\right)$ as the panel elevation angle moves from 45 degrees to 60 degrees. This is likely due to a change in flow separation over the panel, in which at 60 degrees the panel acts much more as a blunt object opposing the flow, causing pressure drag behind the panel. In turn, the lift increases due to a decrease of downward pressure acting on the surface of the panel, due to less exposed area in the $\mathrm{XY}$ plane. The increased drag in the X-direction contributes to the rise of bending torque in the Y-axis, as seen in Figure 6.5.

It is recommended that panel elevation angles remain below 60 degrees in order to substantially decrease the force and moments on the solar panel. Fortunately, high elevation angles are only used when the sun is low in the sky, only indirectly shining sunlight on the panel, meaning that high elevation angles can be most easily removed since they are the least efficient at collecting energy. 


\subsection{Effect of Varying Panel Azimuth Angle}

A panel submersed in wind flow at 40 kilometers per hour was situated in the center of the rooftop, with the rooftop positioned at the zero degree mark and the panel angle at 45 degrees. The panel yaw was varied from 0 degrees down to 180 degrees.

In Figure 6.6 below, a composite plot of $F_{x}, F_{y}$, and $F_{z}$ is shown displaying the mean average of each time series sample as data points for each wind direction.

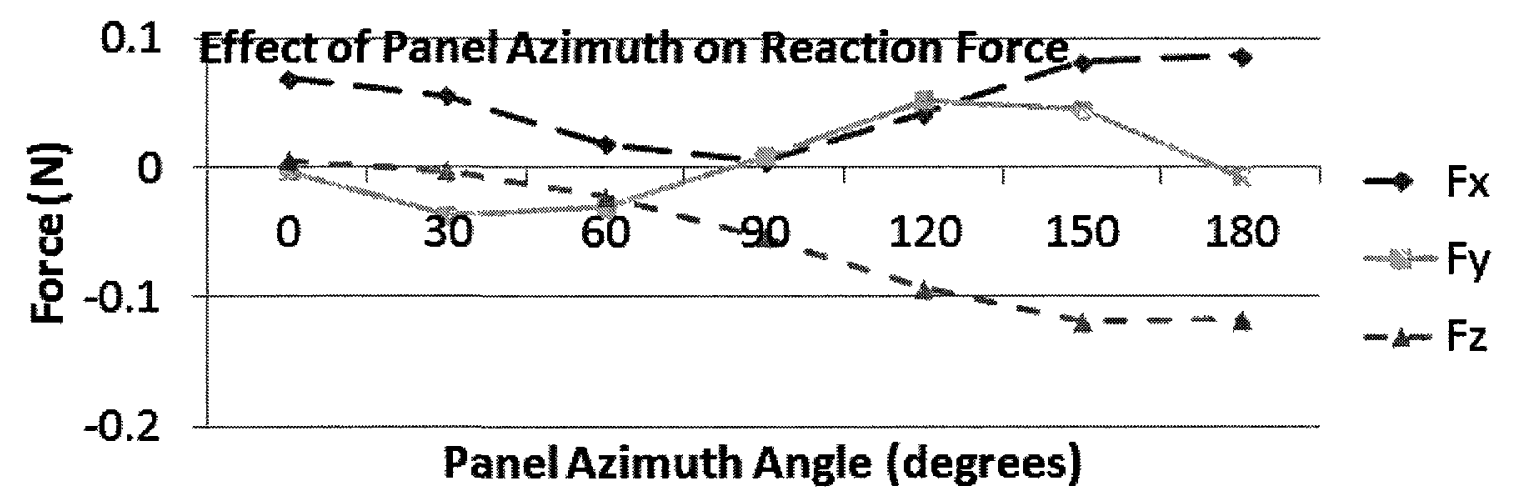

Figure 6.6: Panel azimuth angle reaction forces

In Figure 6.7 below, a composite plot of $M_{x}, M_{y}$, and $M_{z}$ is shown displaying the mean average of each time series sample as data points for each wind direction.



Figure 6.7: Panel azimuth angle reaction moments 


\subsubsection{Significance of Panel Azimuth Angle}

Very clear trends were observed when varying the panel azimuth angle in the wind tunnel. To review, the panel forces and moments were measured at 7 different azimuth angles varying from 0 degrees to 180 degrees. In all cases, the forces and moments appear to follow a sinusoidal trend, as shown respectively in Figure 6.6 and Figure 6.7.

The drag in the X-direction is shown to decrease as the azimuth approaches 90 degrees, and then increase as the azimuth approaches 180 degrees. This correlates with the change in the panel's exposed area in the YZ-plane. The panel reaction force in the Y-direction increases as the azimuth approaches 45 degrees, decreases approaching 90 decrease, and then increases and decreases as the azimuth approaches 135 degrees and 180 degrees, respectively. This is expected since the drag force in the $\mathrm{X}$-direction will contribute a component of its force into the $\mathrm{Y}$-direction as the panel area in the $\mathrm{XZ}$ plane increases and decreases twice in the azimuth revolution. The lift experienced on the panel is shown to increase as the panel swings from 0 to 180 degrees. This is expected since as the azimuth angle changes, the downward force on the panel due to the panel elevation angle is experienced less. Past 90 degrees the elevation angle at the rear of the panel begins to push the panel upwards. This force, combined with the change in dynamic pressure change above, account for the high lift force.

The moment torques experienced by the panel also showed similar trends as shown in Figure 6.6. While the torque in the Z-axis did not vary significantly (due to a balance of exposed area on each side of the axis), torque in the Y-axis was shown to decrease as the azimuth approached 90 degrees, and then increase as the azimuth moves towards 180 degrees. This correlates perfectly with the azimuth trend seen in $F_{x}$, which is the main cause for bending in the $\mathrm{Y}$-axis. Interestingly, torque in the 
$\mathrm{X}$-axis followed a trend that showed $M_{x}$ decrease approaching 45 degrees, increase approaching 135 degrees, and then decrease approaching 180 degrees. This pattern may be explained by wind flow pressing down more on the side of the panel it reaches first, thus slowing the wind that is pressing on the opposite side. This would create the bending observed in the $\mathrm{X}$-axis.

In terms of exposure limits, the panel will see the highest lift forces when the azimuth is 180 degrees away from the wind flow direction. The drag forces in the $\mathrm{X}$-direction will be highest when the panel is facing away from the wind direction, and the drag in the Y-direction is highest when the panel azimuth is half way between pointing into or away from the wind and being completely sideway into the wind.

\subsection{Effect of Varying Wind Direction}

A panel submersed in wind flow at 40 kilometers per hour was situated in the center of the rooftop, with the panel angle at 45 degrees. The rooftop was varied from 0 degrees down to 45 degrees while the panel remained at 0 degrees.

In Figure 6.8 below, a composite plot of $F_{x}, F_{y}$, and $F_{z}$ is shown displaying the mean average of each time series sample as data points for each building orientation.

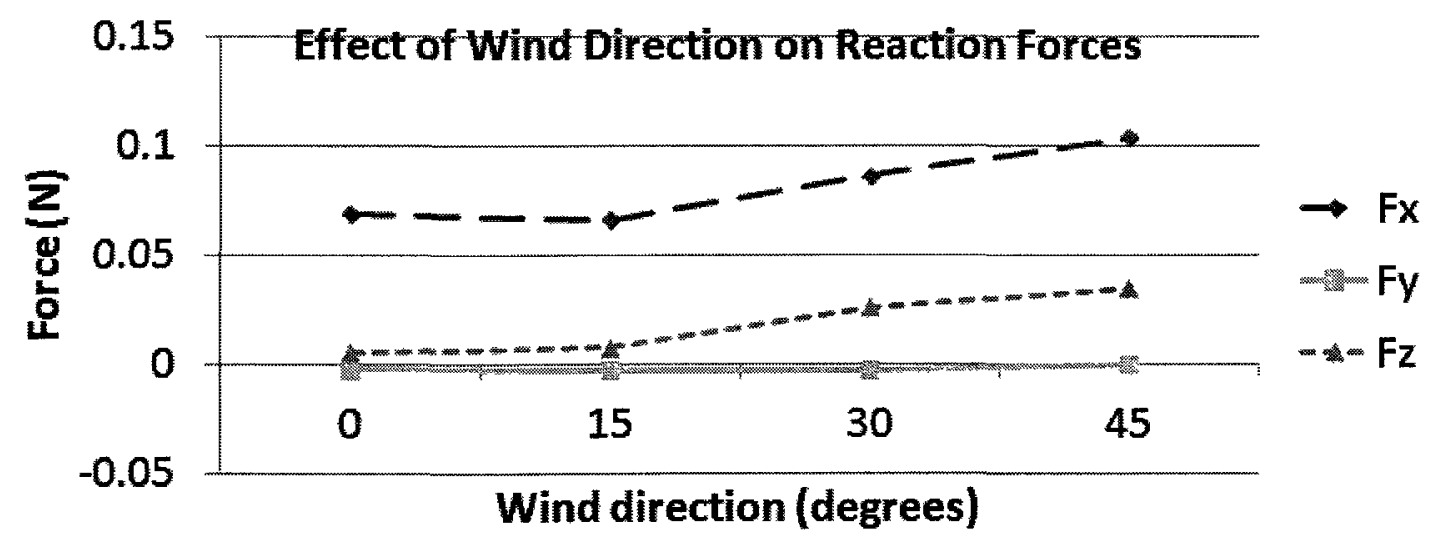

Figure 6.8: Wind direction reaction forces 
In Figure 6.9 below, a composite plot of $M_{x}, M_{y}$, and $M_{z}$ is shown displaying the mean average of each time series sample as data points for each building orientation.

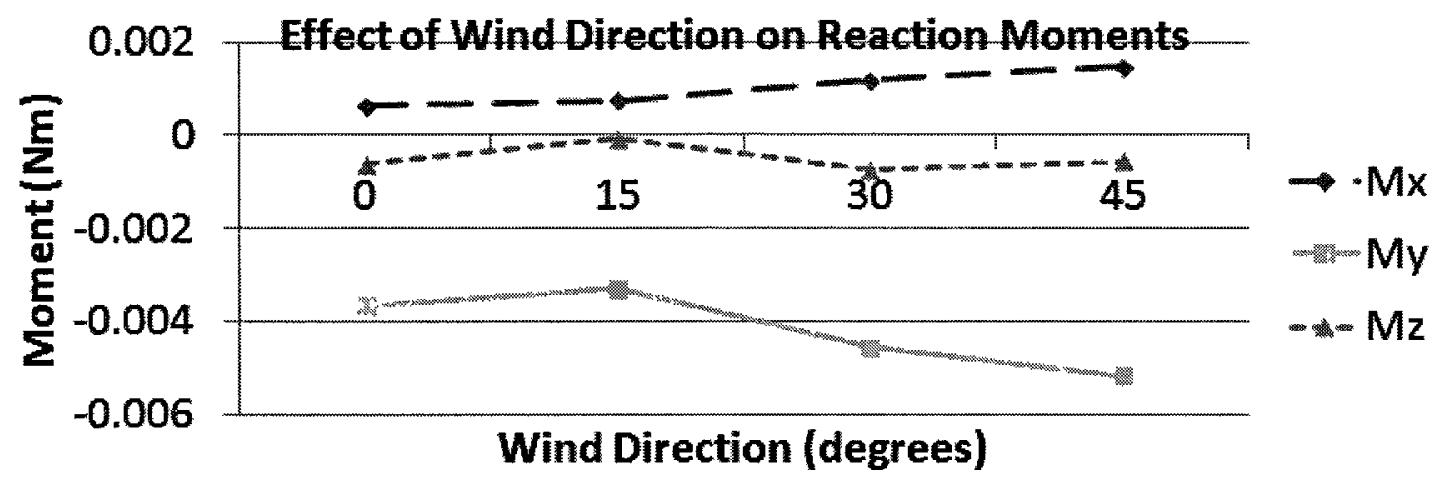

Figure 6.9: Wind direction reaction moments

\subsubsection{Significance of Wind Direction}

The effect of changing the wind direction, accomplished by rotating the building in the wind tunnel, was monitored on the solar panel model. The forces measured, shown in Figure 6.8, show that while the force in the Y-direction remained relatively unchanged, both the force in the $\mathrm{X}$ and $\mathrm{Z}$ direction were shown to increase substantially as the wind direction approached 45 degrees. It is likely that the effect of the boundary layer separation of the building' leading edge on the panel model was lessened. With wind striking the building closer to the rooftop corner, which is farther away from the panel model, the boundary layer likely reattaches closer to the solar panel. Instead of the being diverted up and over the panel (which would create more lift and less drag), the effect is that the wind flow comes back down on the panel more, creating higher drag in the X-direction and pushing the panel into the rooftop.

Correlating to the force in the X-direction, as seen in Figure 6.9, the torque in the $\mathrm{Y}$-axis is shown to increase approaching a wind direction of 45 degrees, as the drag force in the X-direction increases. This is expected since the drag force is the main 
contributor to bending moments in the $\mathrm{Y}$-axis. Bending moments in the $\mathrm{X}$ and $\mathrm{Z}$ axis are shown to slightly increase or decrease as the wind direction changes. This is likely due to the unsymmetrical separation of the wind boundary layer as it hits the corner of the building off to one side first, instead of separating direction in front of the panel on the buildings leading edge.

It is less likely that the panel will experience lift problems when wind approaches from a building's corner, however this will create higher drag forces and thus bending moments on the panel.

\subsection{Effect of Varying Panel Rooftop Locations}

A panel submersed in wind flow at 40 kilometers per hour was situated at various locations on the rooftop, with the panel angle at 45 degrees. The rooftop remained at 0 degrees while the panel remained at 0 degrees. The results are presented below.

In Figure 6.10 below, a composite plot of $F_{x}, F_{y}$, and $F_{z}$ is shown displaying the mean average of each time series sample as data points for each panel position.

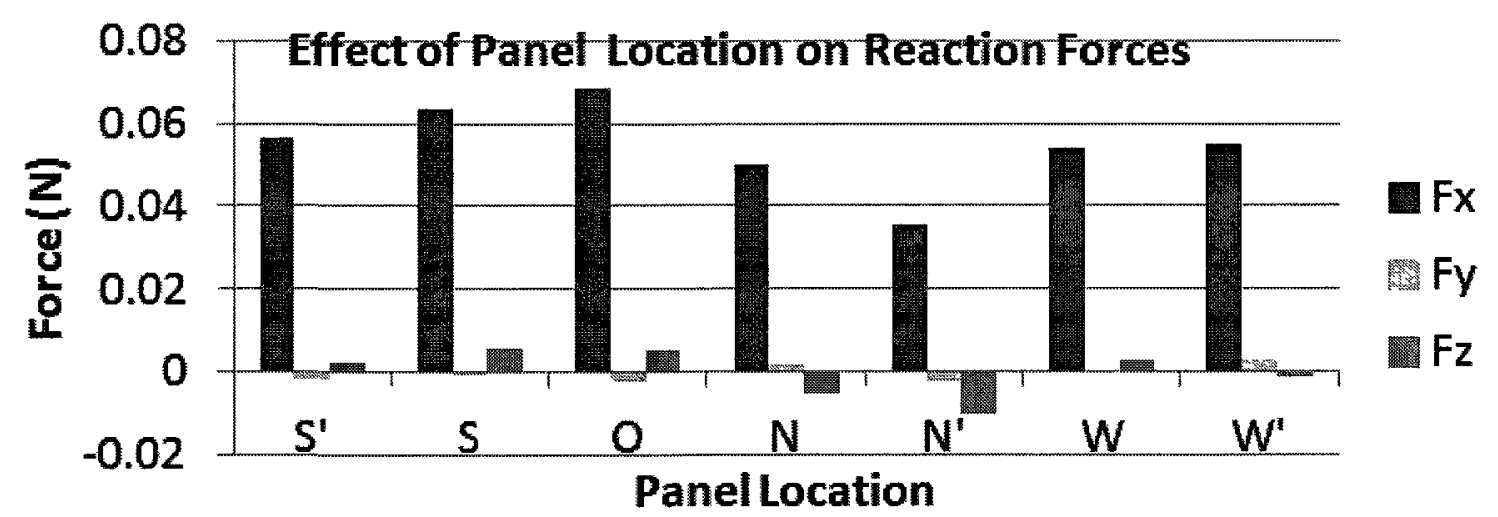

Figure 6.10: Panel position reaction forces

In Figure 6.11 below, a composite plot of $M_{x}, M_{y}$, and $M_{z}$ is shown displaying the mean average of each time series sample as data points for each panel position. 




Figure 6.11: Panel position reaction moments

\subsubsection{Significance of Panel Rooftop Location}

A solar panel can be placed anywhere on a rooftop. With wind flowing over the building, different locations on the roof will exert different forces on the solar panel. During the wind tunnel experiment, locations were monitored one and two spaces ahead, behind, and beside the building center O. As seen in Figure 6.10, the location did not affect the force in the Y-direction. However, a visible trend is seen regarding the force in the X-direction. Drag is shown to be lowest close to the leading edge of the building. As the panel is shifted downstream toward position $\mathrm{O}$, the drag increases. Moving downstream of position $\mathrm{O}$, the drag force has a decreasing trend. This may be explained by the boundary layer separation at the leading edge of the building. Flow diverted up and over the panel would reduce its exposure to drag, and great a change in dynamic pressure overhead that would pull the panel upwards. This correlation between lift and drag is also clearly visible in Figure 6.10. Another trend noticed is the increase of lift force in the Z-direction as the panel is positioned closer to the side of the building. This is likely due to edge effects along the side of the building. Therefore, to decrease the drag force on the panel, the panel should be close to the leading edge of the building along its centerline, however this not only 
increases the lift force, but is also subjective to the relative direction of the wind on the building (should it happen to change and make the panel "appear" to be near a side edge instead of the leading edge). Also, as shown in Figure 6.11, positioning of the panel closer to the leading edge significantly reduces the panel torque experienced in the Y-axis.

\subsection{Effect of Nearby Panels}

A panel submersed in wind flow at 40 kilometers per hour was situated in the center the rooftop, with the panel angle at 45 degrees. The rooftop remained at 0 degrees while the panel remained at 0 degrees. A second panel was placed at various locations on the rooftop, and its effects on the first panel are presented below. Although not addressed in this thesis, locating a panel ahead of another panel could cause cooling problems for the downstream panel, and should be investigated in the future.

In Figure 6.12, a composite plot of $F_{x}, F_{y}$, and $F_{z}$ displays the mean average of each time series sample as data points for each dummy panel position.



Figure 6.12: Adjacent panel position reaction forces

In Figure 6.13, a composite plot of $M_{x}, M_{y}$, and $M_{z}$ displays the mean average of each time series sample as data points for each dummy panel position. 


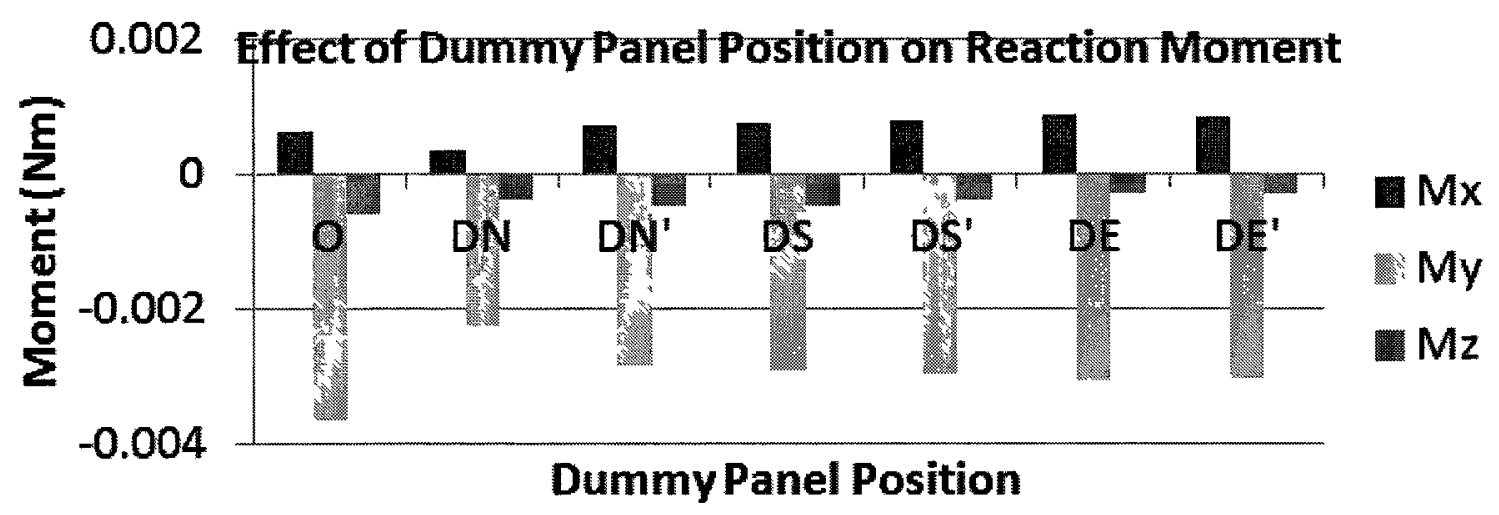

Figure 6.13: Adjacent panel position reaction moments

\subsubsection{Significance of Nearby Panels}

With the active panel in the building center at position $\mathrm{O}$, six different test cases were examined. A dum $M_{y}$ panel was placed one or two spaces behind, beside, or in front of the active panel. As seen in Figure $6.12, F_{x}$ was largely affected with the placement of a panel one position in front of it. This is likely due to the dummy panel separating the flow and diverting it overhead. The increased flow over top of the active panel would create higher dynamic pressure, thus creating more lift in the negative Z-direction as shown in Figure 6.12. This in turn also marks a significant decrease in the torque on the panel in the $\mathrm{Y}$-axis. The remainder of the test conditions did not show a significant change. Thus, when choosing where to place two panels on a rooftop, it is best if they are not side by side, but one in front of the other as close as possible without infringing the sun exposure.

\subsection{Effect of Panel Submersion in a Field of Panels}

A panel submersed in wind flow at 40 kilometers per hour was situated in the center the rooftop, with the panel angle at 45 degrees. The rooftop remained at 0 degrees 
while the panel remained at 0 degrees. Clusters of panels of various sizes were placed on the rooftop. Their effects on the first panel are presented below.

In Figure 6.14 below, a composite plot of $F_{x}, F_{y}$, and $F_{z}$ is shown displaying the mean average of each time series sample as data points for each cluster arrangement.

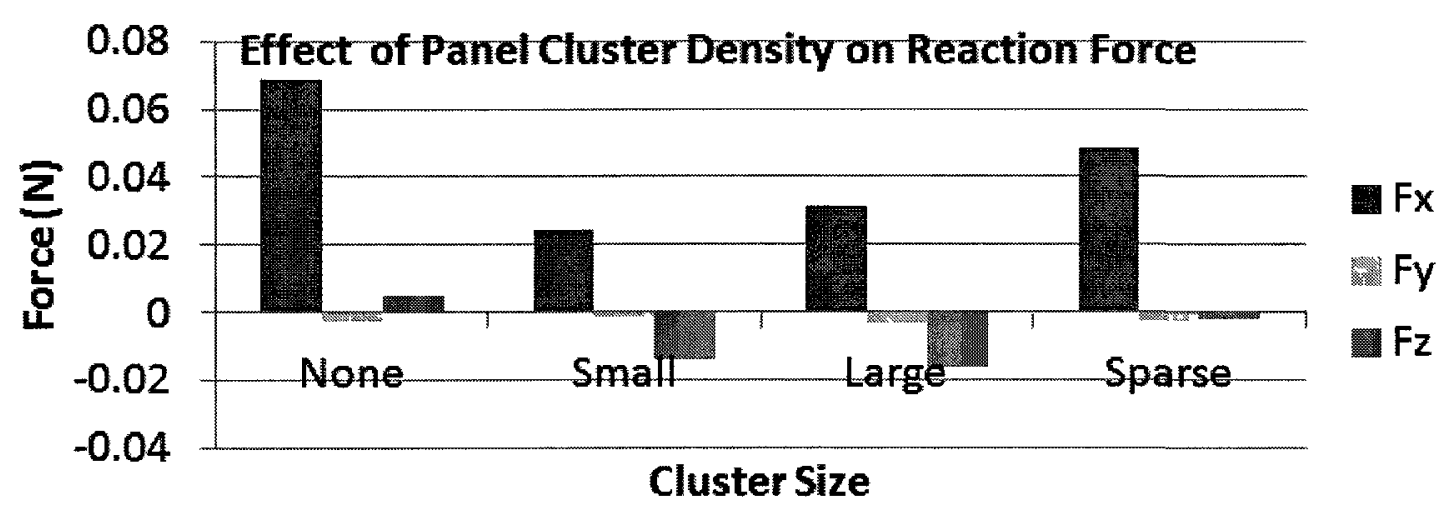

Figure 6.14: Panel field reaction forces

In Figure 6.15 below, a composite plot of $M_{x}, M_{y}$, and $M_{z}$ is shown displaying the mean average of each time series sample as data points for each cluster arrangement.

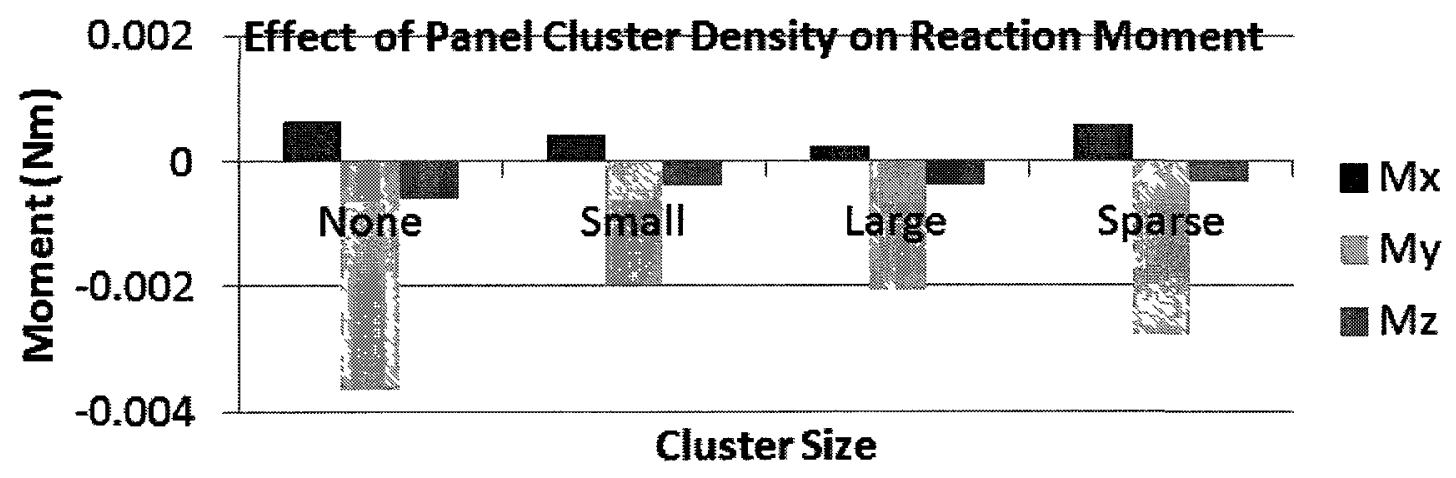

Figure 6.15: Panel field reaction moments 


\subsubsection{Significance of Panel Fields}

The four different cases tested with the active panel in the building center at position O were one row of surrounding panels, two rows of surrounding panels, and then a sparse mix between the two. As shown in Figure 6.14, $F_{y}$ is unaffected throughout. The highest $F_{x}$ is experienced when there are no surrounding panels. It appears that having panels immediately surrounding the active panel creates a lower $F_{x}$ and higher negative $F_{z}$. This may be due to wind flow being projected over top of the active panel, reducing its direct exposure in the $\mathrm{X}$-direction while at the same time increasing flow rate over top, which would thus increase dynamic pressure and pull the panel upwards in the negative Z-direction. The larger field would therefore allow the flow separation to reattach, raising the $F_{x}$ slightly. The sparse panel distribution supports this theory, as it would expose the panel to more direction wind flow in the X-direction, while still diverting some flow overhead and creating lift in the Zdirection. It can thus be said that if drag force is a problem, having a close panel cluster is best. As such, moment in the $\mathrm{Y}$-axis is primarily affected by $F_{x}$. Thus, as shown in Figure 6.15, the bending moment is most reduced in a tight cluster of panels. If panel lift force is a problem, having a larger distribution of panels is best. 


\section{Chapter 7}

\section{Computational Simulation Setup}

Using CFD to compliment the wind tunnel experiment was motivated by numerous benefits. It will give a clear visual representation of the physics of fluid flow, and provide approximate magnitudes of panel load data to assist with selection of wind tunnel data acquisition hardware. Ansys CFX Version 12 was used for the simulation. The geometry for the simulation was developed in Ansys ICEM. The setup for this simulation was designed to replicate the wind tunnel experiment. This section will examine the geometry and meshing used to implement the described solution method. The next subsection will first provide an overview of the result obtained from attempting to implement randomized upstream turbulence.

\subsection{Introducing Randomized Turbulence}

Although CFD code developed in FORTRAN was further developed, it is not ready to be used for implementing upstream atmospheric boundary layer turbulence. Continual research is required. With further work, modeling atmospheric flow will be specifically enhanced. For example instead of the lengthy process of modeling the entire upstream flow of a solar panel or wind turbine with a few kilometres of forest ahead of it, the code will calculate the flow developed over the upstream ground much 
faster. This upstream data can then be imported into a commercial package such as Ansys CFX as initial conditions of the flow. This will save a number of computation resources, as it reduces the domain size, and has more accurate input flow. There are several next steps that will ensure the current FORTRAN code goes to the proper phase where it is applicable. The current code exists for flows in all directions; however in some conditions restrictions could occur. For example, flow over a surface like the ground. Another boundary condition that should be included is that of obstacles in the flow, such as trees in a field. Modelling these boundary conditions would allow a wider variety of upstream flow to be simulated, such as boundary layer development and its turbulence.

In lieu of modeling the development of the atmospheric boundary layer, the correct fully developed boundary layer profile will be directly setup in the initial conditions.

\subsection{Domain and Boundary Condition Setup}

Using Ansys Workbench, the geometry and mesh are loaded onto a CFX system. Then, two types of simulations were run. The first is a steady Reynolds Averaged Navier Stokes analysis. This is used for domain sizing and grid convergence analysis, and for establishing initial conditions to run the second type of simulation. The second type is a Large Eddy Simulation that will use the converged domain and grid to compute and render vortexes being shed off domain flow obstructions such as the panel. A description of the RANS and LES models along with background CFD information can be found in Appendix D.

\subsubsection{Boundary Conditions}

The boundaries in the domain include the inlet, outlet, walls, ground, ceiling, and panel. The ground, ceiling, and panel are setup as no-slip wall conditions. The outlet 
is defined with a zero pressure difference and the walls are set up with symmetry. Symmetrical walls restrict the system to two dimensions during simulations, and can be thought of as having slip free walls as well.

The inlet is defined as subsonic with a velocity profile matching a power law distribution of the atmospheric boundary layer. The roughness coefficient matching the spires in the wind tunnel was found by using wind tunnel boundary layer data. The wind tunnel boundary layer at a mean velocity of $40 \mathrm{~km} / \mathrm{h}$ is shown below in Figure 7.1.

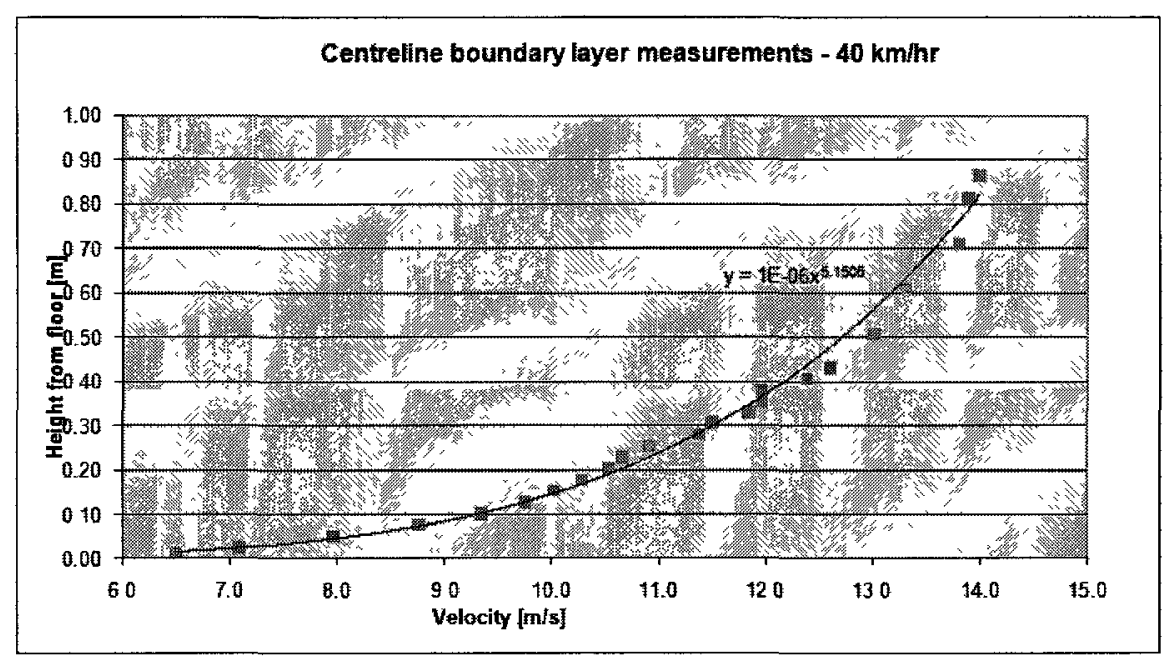

Figure 7.1: Wind tunnel simulated atmospheric boundary layer [17]

Using Microsoft Excel's trend line function, a power law curve was fitted to the graph. Equating this trend line to the power law equation, and taking a known data point in the boundary layer, the roughness coefficient was found to be 0.19415 . Using this information, the full power law function was defined in the inlet as:

$$
U=U_{\text {ref }}\left(\frac{Z}{Z_{\text {ref }}}\right)^{\alpha} \quad=12.9396 \mathrm{~m} / \mathrm{s}\left(\frac{z}{0.5334 m}\right)^{0.19415}
$$




\subsubsection{Domain and Solver Setup}

For the RANS test, a steady state domain using an SST turbulence model was used with an upwind advection scheme. Medium turbulence was assigned to the inlet. The default flow medium of sea level air was used. Solver control was set up to run 1000 iterations, running 4 local processors in parallel. Monitor points for the panel's force and moment were introduced to allow their data to be analyzed after the simulation.

For the LES simulation, a transient domain using 20,000 time steps for 2 seconds was used. The Smagorinsky modeling method was used, with a constant of 0.1. Up to 3 coefficient loops were permitted for each time step. For the initial domain velocities, the converged RANS solutions were used. Central differencing was set as the advection scheme, and second order backward Euler as the transient scheme. The transient results were output for every 100 time steps to save hard drive space. The transient results were set to monitor the Courant number so that the amount of dissipation could be recognized during and after the simulation, as well as the monitoring of $F_{x}, F_{z}$, and $M_{y}$. The simulation was performed over the duration of a week on a networked computer cluster consisting of twenty processors.

\subsection{Domain Sizing}

Due to the relative simplicity of the geometry, it was decided early on that a twodimensional computer simulation would be performed. This geometric simplicity is seen as a cross section along the length of the test section remaining relatively constant, with the exception being at the side edges of the building, the side edges of the panel model, and the wind tunnel walls. Although the domain is presented as two dimensional, the setup of the structured grid is more accurately described as a quasi-3D domain, which is a $3 \mathrm{D}$ domain acting as $2 \mathrm{D}$. This is done by making the thickness of the domain in the y-direction only one cell wide, with the cell set at 1 
inch in length allowing for easy result extrapolation to represent a 3D solution. The resulting expanded domain can be seen in Figure 7.2 below.

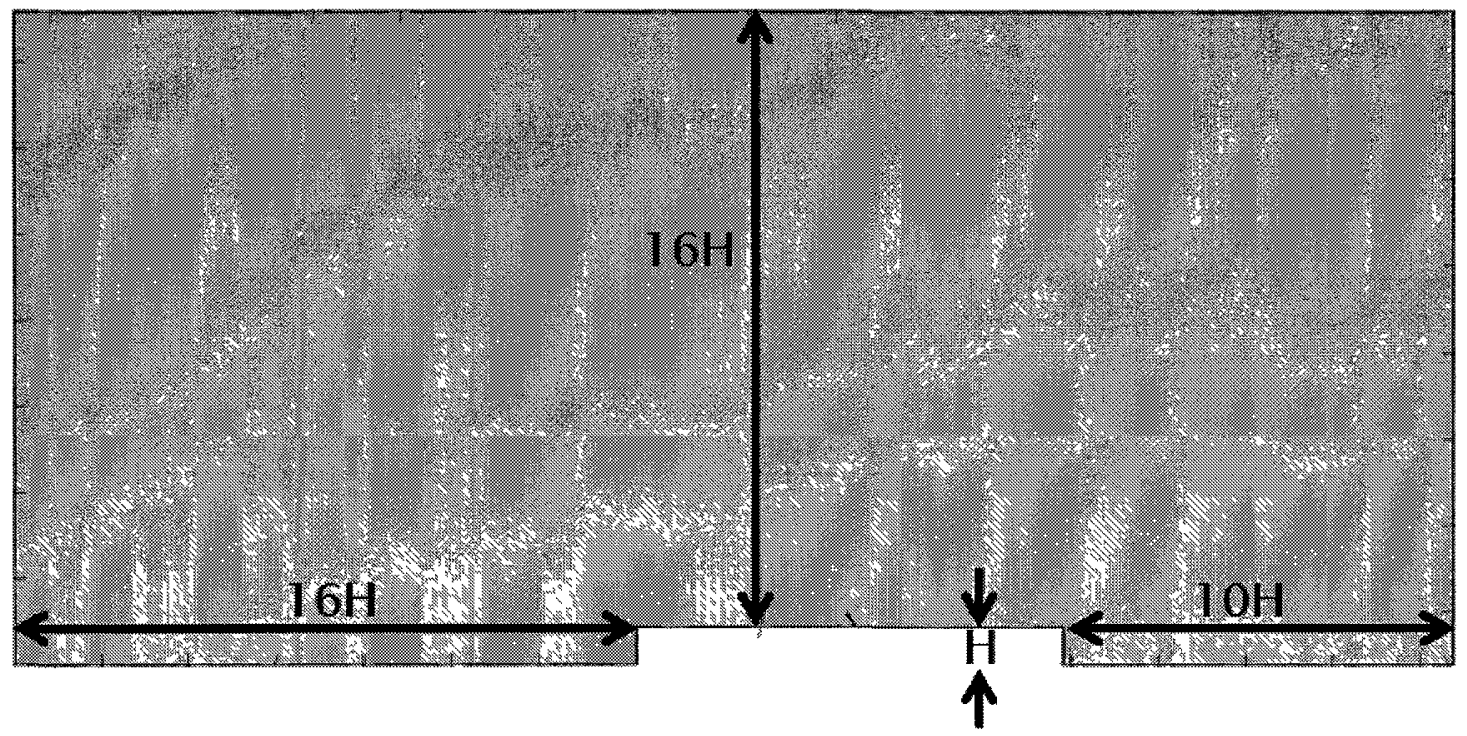

Figure 7.2: Final domain size

The domain was based on the cross-section of the wind tunnel building. The domain distance upstream and downstream needed to be determined, as well as the domain height above the building. Iterative increases of various intervals of the building height were used to extend the domain. A rough mesh was used so quick iterations could be performed. The drag force was measured on the panel. Since the panel cross section reveals the center panel gap, force was able to be measured for the top and bottom half of the panel. These drag forces were plotted against iterations of domain size in terms of building height intervals. The domain downstream of the building was first checked. The results can be shown below in Figure 7.3. 


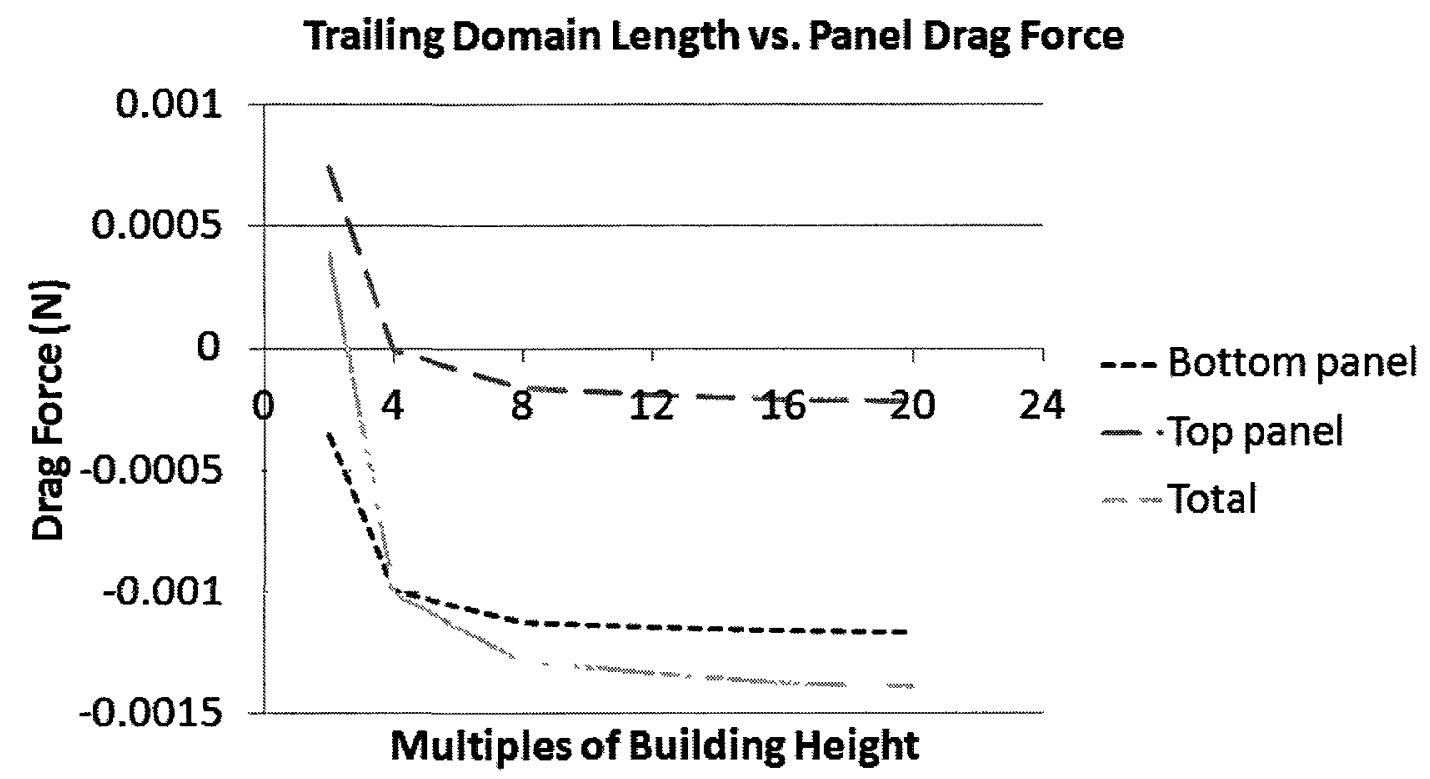

Figure 7.3: Downstream domain sizing

As seen, the force on each half of the panel as well as the total panel converged to a specific value once the downstream domain distance reached approximately 16 times the height of the building. Second, the domain above the building was checked. The results can be shown below in Figure 7.4. 
Domain Height vs. Panel Drag Force

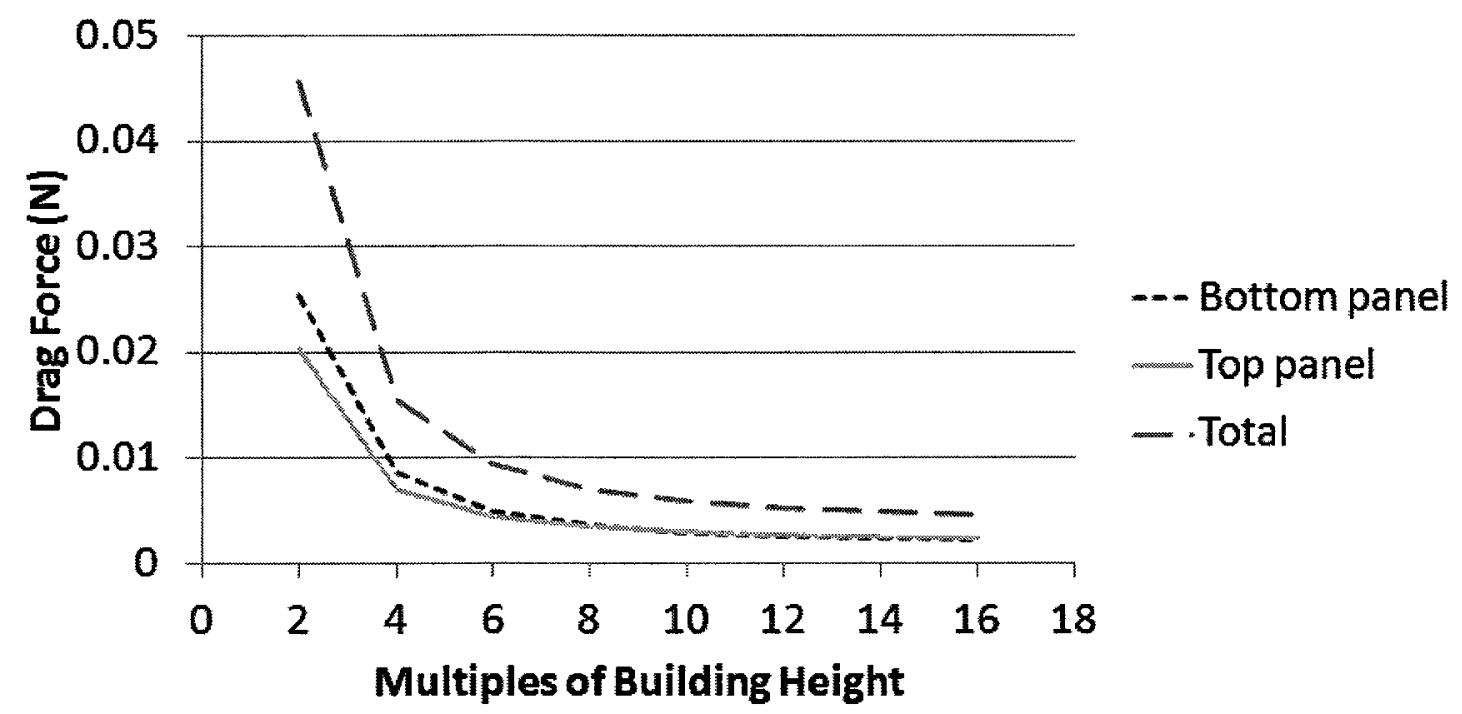

Figure 7.4: Domain ceiling sizing

The force on each half of the panel as well as the total panel converged to a specific value. Again, this occurred once the downstream domain distance reached approximately 16 times the height of the building. The domain upstream of the building was checked third. The results can be shown below in Figure 7.5. 


\section{Inlet Domain Length vs. Panel Drag Force}

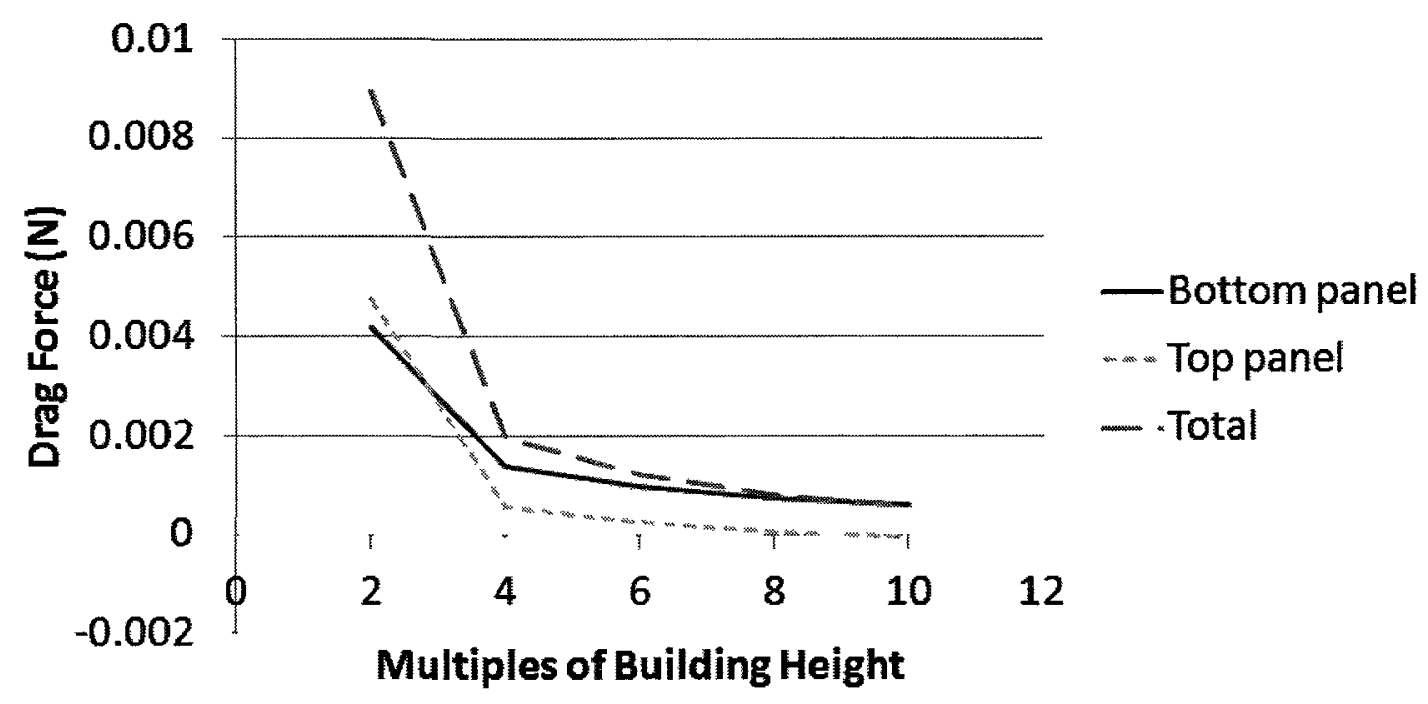

Figure 7.5: Upstream domain sizing

The force on each half of the panel as well as the total panel converge to a specific value. Again, this occured once the downstream domain distance reached approximately 10 times the height of the building. The domain convergence study showed that the domain size will be 16 times the building height downstream, 10 times the building height upstream, and 16 times the building height above the building. With domain size determined, the next step was to determine a proper mesh distribution.

\subsection{Grid Density}

The flow domain contains areas where the flow changes drastically. Such locations could include friction along the domain walls, and possible boundary layer separation areas such as the building and panel leading edges. The rate at which distance changes the flow is much smaller than other locations in the domain. Finding a correct distribution of grid nodes will allow the desired flow characteristics to be properly resolved, namely the flow around the rooftop panels. The initial grid mesh 
used with the converged domain can be seen below in Figure 7.6

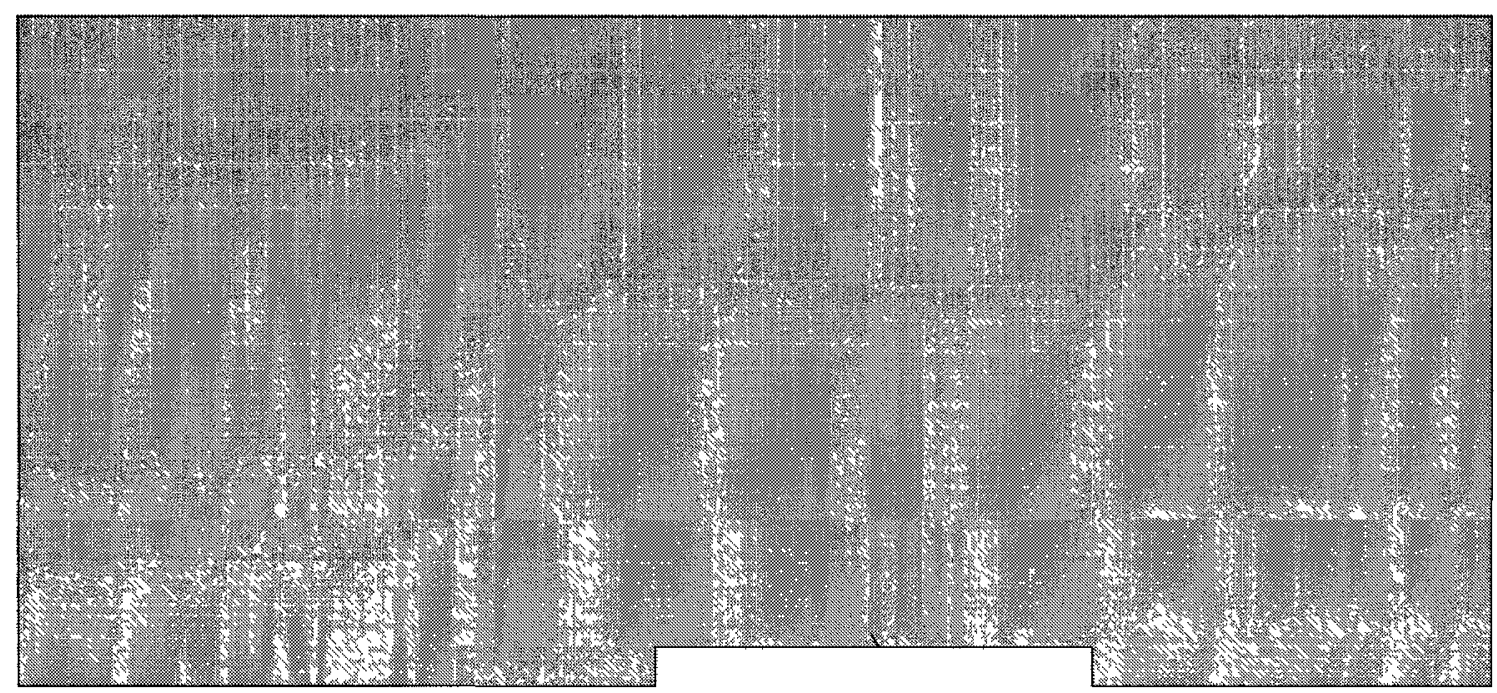

Figure 7.6: Initial mesh with converged domain

The method used for increasing grid density to the optimum distribution of nodes involved starting far away from the panels and moving inwards. As the density was increased, the drag force on the bottom and top half of the panel was measured. Once the drag force measured was shown to have converged, that section of the domain's mesh was considered optimized. This process was repeated until every area of the mesh had its density increased to the point of drag force convergence. A plot of the various steps of convergence can be found below in Figure 7.7. 


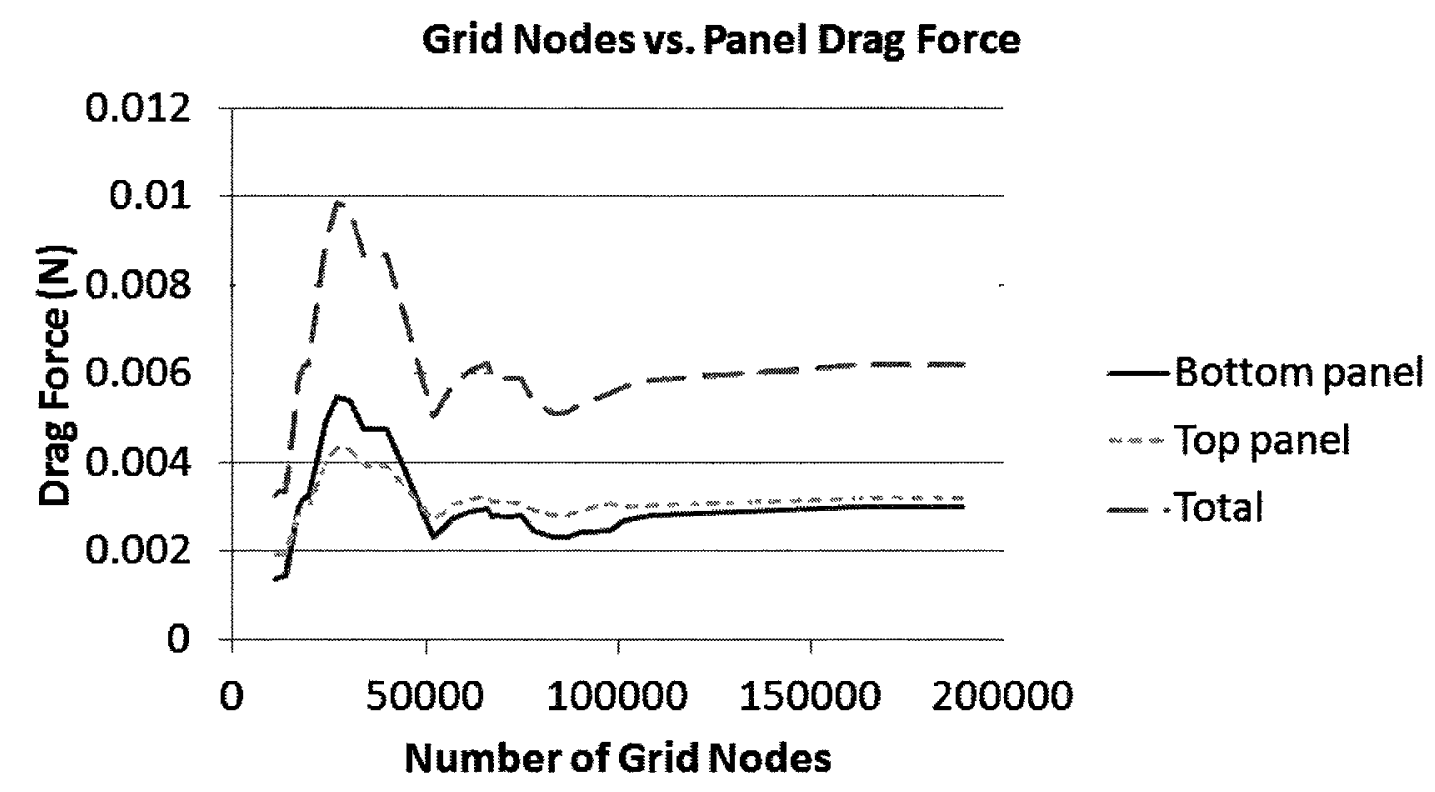

Figure 7.7: RANS grid convergence

As can be seen in the above graph, the force measured on the panel varied but eventually approached a converged value after various areas of the domain were systematically altered with a denser mesh.

This converged mesh used with the sized domain is optimized to provide the most efficient computation that will still produce the desired result accuracy. An image of the converged grid and domain is shown below in Figure 7.8. 


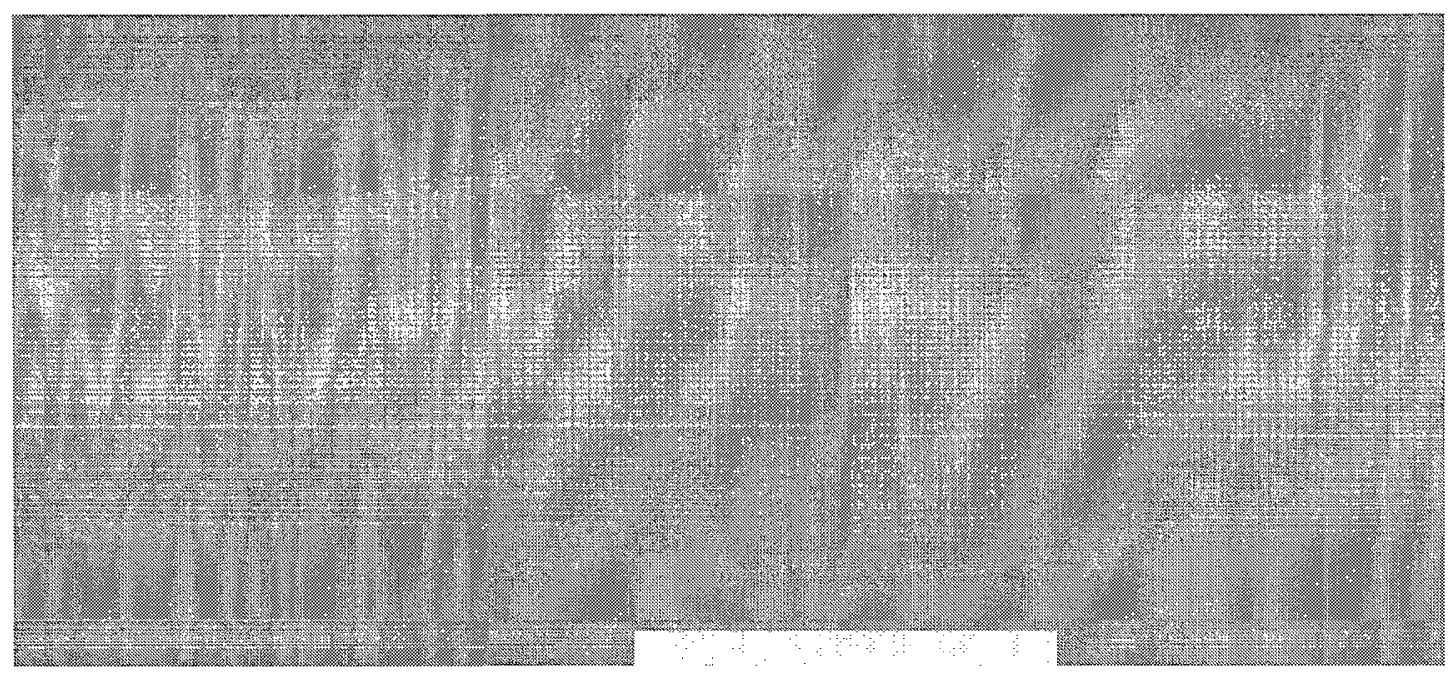

Figure 7.8: RANS converged mesh

Additionally, a grid convergence study was also performed using a transient LES setup, in which the osciallating forces and moment were averaged, and plotted against the number of nodes in the mesh. A plot of this convergence can be found below in Figure 7.9 .

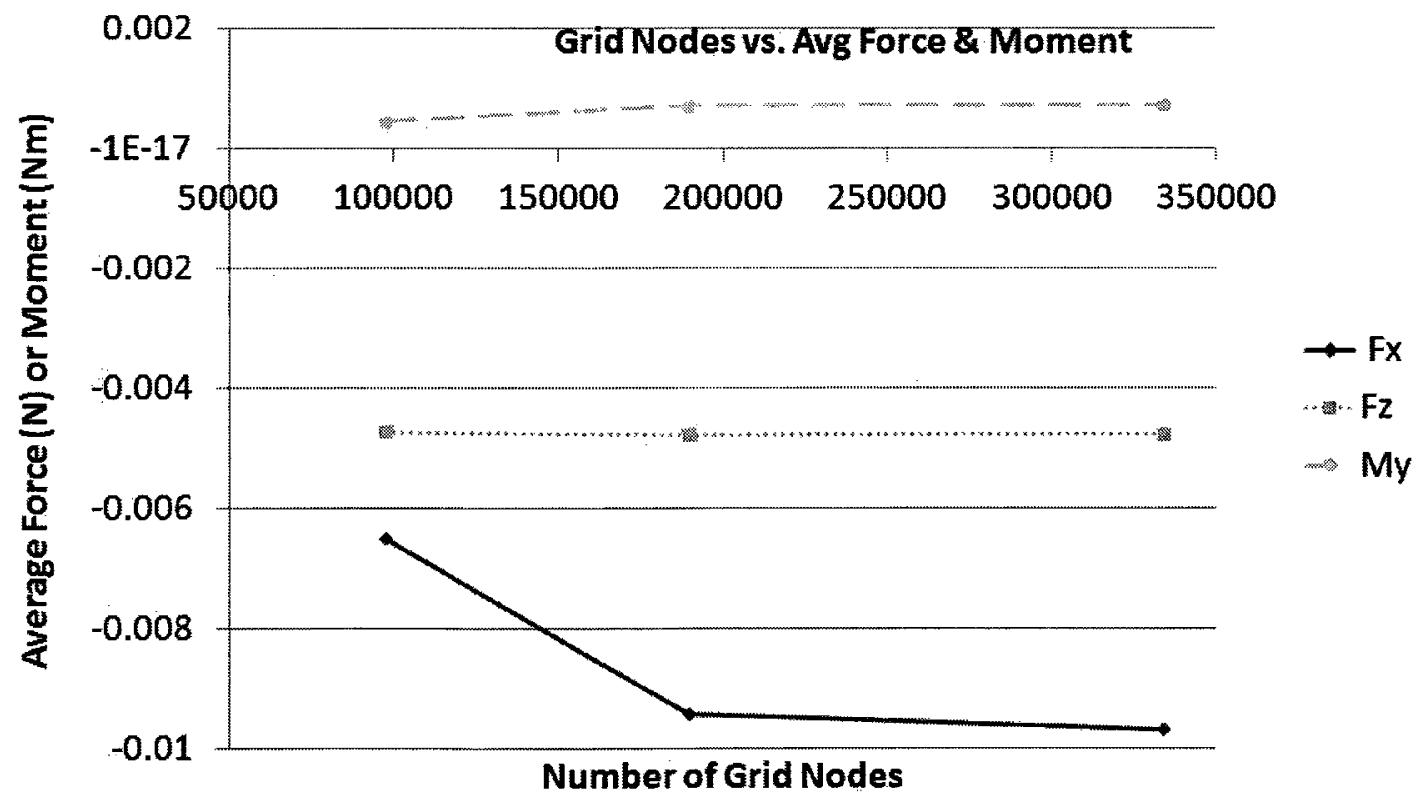

Figure 7.9: LES grid convergence 
As can be seen in the above graph, the forces and moment measured on the panel quickly converge by the second iteration. Therefore the grid density of approximately 190,000 nodes is sufficient for both RANS and LES setups. 


\section{Chapter 8}

\section{Computational Results and Discussions}

Computational results using the commercial package CFX are presented and discussed, giving a representation of the flow simulation being experienced as it travels over the top of the building. Submersion of a solar panel into the flow is used to derive expected forces and moments acting on the panel and to compare to wind tunnel results. The Reynolds Averaged Navier-Stokes simulation is first shown, followed by the Large Eddy Simulation.

\subsection{D LES Forces and Torque}

The first simulation ran over the course of a few hours and was a steady state RANS flow using SST turbulence modelling. To have an idea of how the averaged flow was represented, a plane cut vertically through the panel and building shows a cross section of the flow velocity and pressure distribution, displayed below in Figure 8.1. 


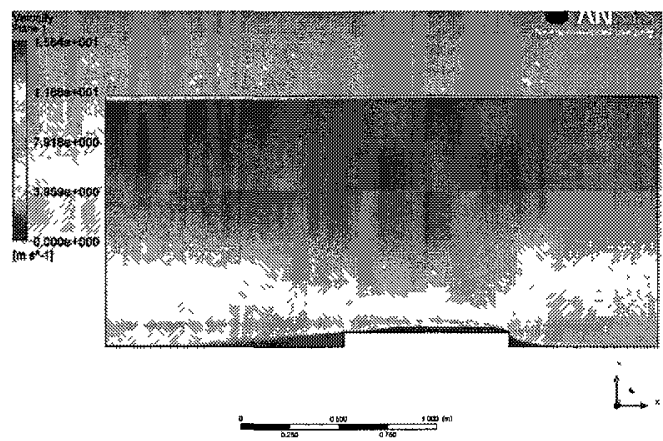

(a) Velocity Distribution



(b) Pressure Distribution

Figure 8.1: Steady state RANS

The initial steady state RANS computation provided an initial understanding of what the flow was experiencing moving over the building. As seen in Figure 8.1, the building rooftop creates a clear separation in the boundary layer. This effect in turn exposes the rooftop solar panel to much lower wind velocities than if it were simply on the ground in the normal atmospheric boundary layer flow. The pressure distribution shown in Figure 8.1 provides a clear picture of the pressure drag experienced by the solar panel. From this image it is clear that moving the solar panel toward the leading edge of the building would create equal pressure on the front and rear of the panel, reducing its drag in the X-direction. This is of a course a simplification since the unsteady turbulent nature of the flow is not presented. The averaged forces and moments calculated from the RANS simulation are presented in the Figure 8.1 below:

Table 8.1: RANS forces and torque

\begin{tabular}{|c|c|}
\hline $\mathrm{Fx}$ & $0.006193 \mathrm{~N}$ \\
\hline $\mathrm{Fz}$ & $-0.003316 \mathrm{~N}$ \\
\hline $\mathrm{My}$ & $0.0005606 \mathrm{Nm}$ \\
\hline
\end{tabular}

As seen in the results, the wind flow creates drag in the stream wise direction, while pushing the panel down into the building roof top. A torque is also present 
on the panel, attempting to prying the panel downstream. The solution from the RANS flow was used as the initial conditions for the transient LES flow. Without these initial conditions, LES cannot be performed. After the course of a week, the simulation was complete, and every 100 time steps of the simulation was recorded for data analysis. In Figure 8.2 an example of an eddy vortex can be seen shedding off the building leading edge and panel trailing edge.

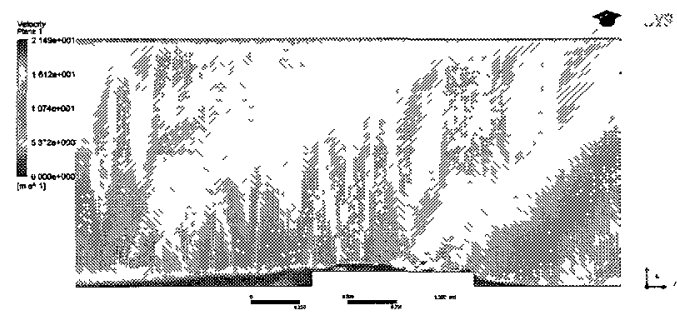

(a) Vortex starting



(c) Vortex after 0.5 milliseconds

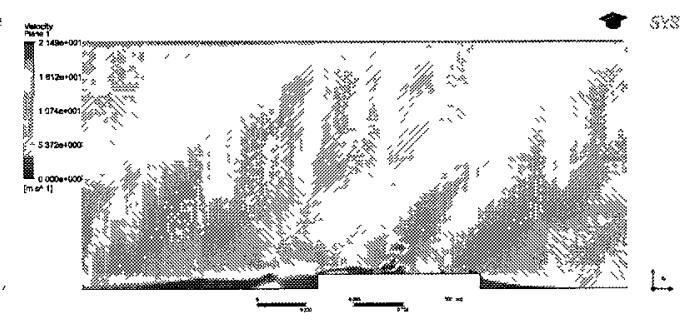

(b) Vortex after 0.25 milliseconds

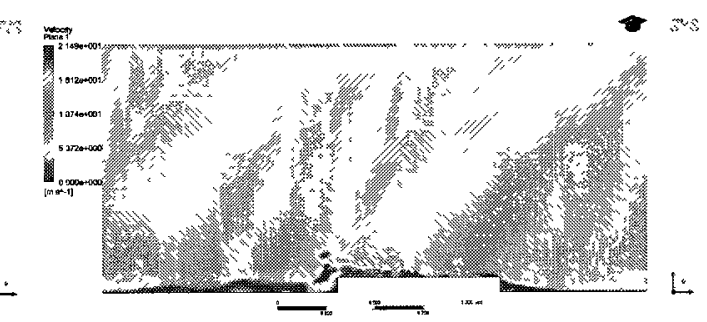

(d) Vortex after 0.75 milliseconds

Figure 8.2: Example of vortex shedding

As seen in Figure 8.2, the boundary layer separation at the leading edge of the building is much more dynamic than the steady state model. A large vortex is seen being shed from the leading edge of the building and passes overhead of the panel, moving on past the building. As the vortex moves downstream, the boundary layer below it is disrupted, creating flow velocity much faster than when examined in the steady state simulation.

This vortex causes fluctuations in the forces and moment experienced on the panel by the air flow. From the monitor points that were setup before the simulation, the 
force fluctuations can be viewed in Figure 8.3.

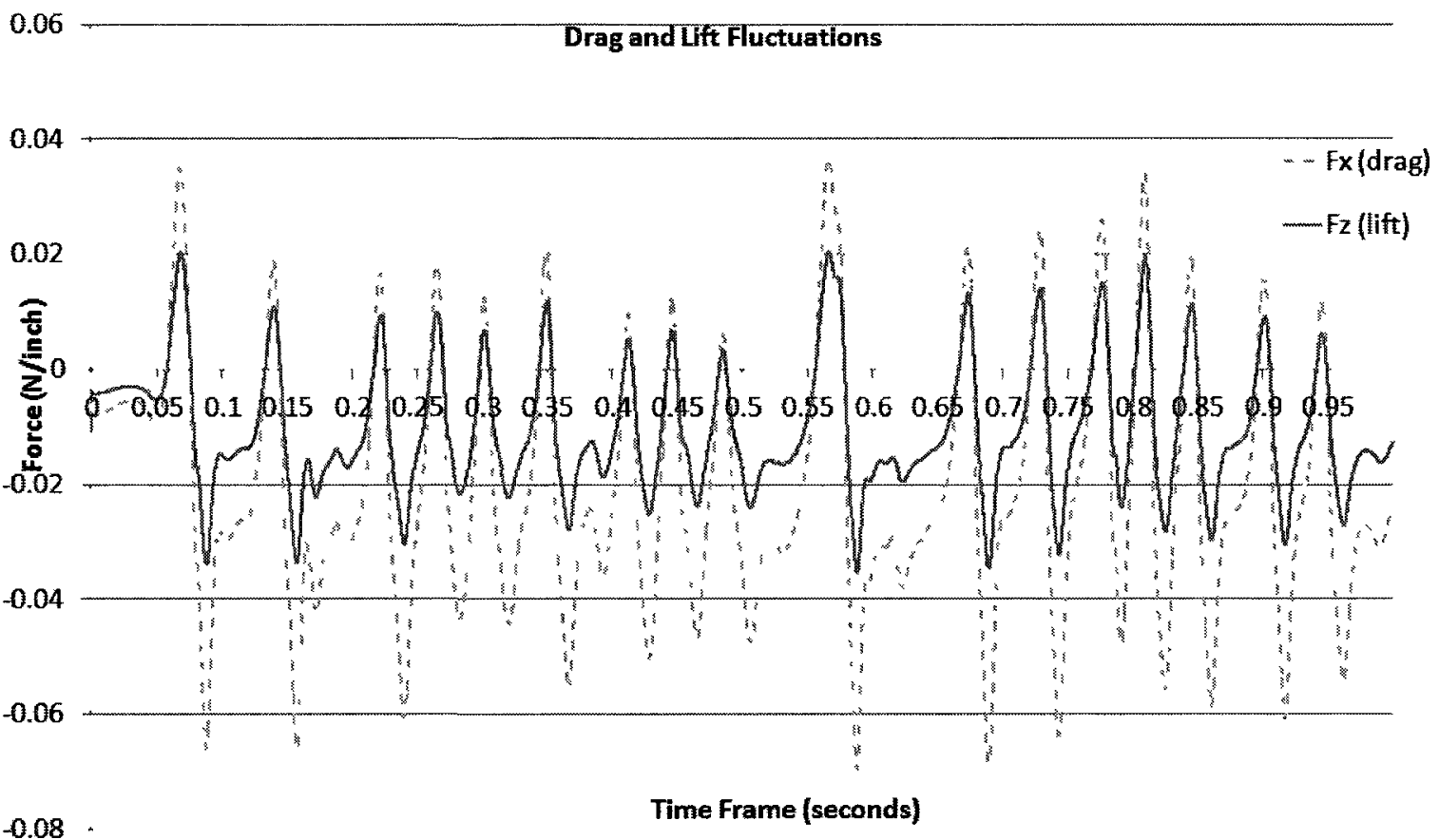

Figure 8.3: Panel forces over time

Over the course of the simulated time, 17 large oscillations occur in both the drag force in the X-direction and the lift force in the Z-direction. The oscillations for lift and drag, which occur at the same moments, are in response to a shed vortex from the leading edge of the building passing overhead of the panel. Between these large oscillations, several small oscillations are also observed. These may be due to smaller vortex shedding off the actual panel itself, because as presented in Figure 3.1, having the panel elevation angle at 60 degrees will induce this vortex shedding.

The same oscillations are noticed in the panel torque seen below in Figure 8.4 


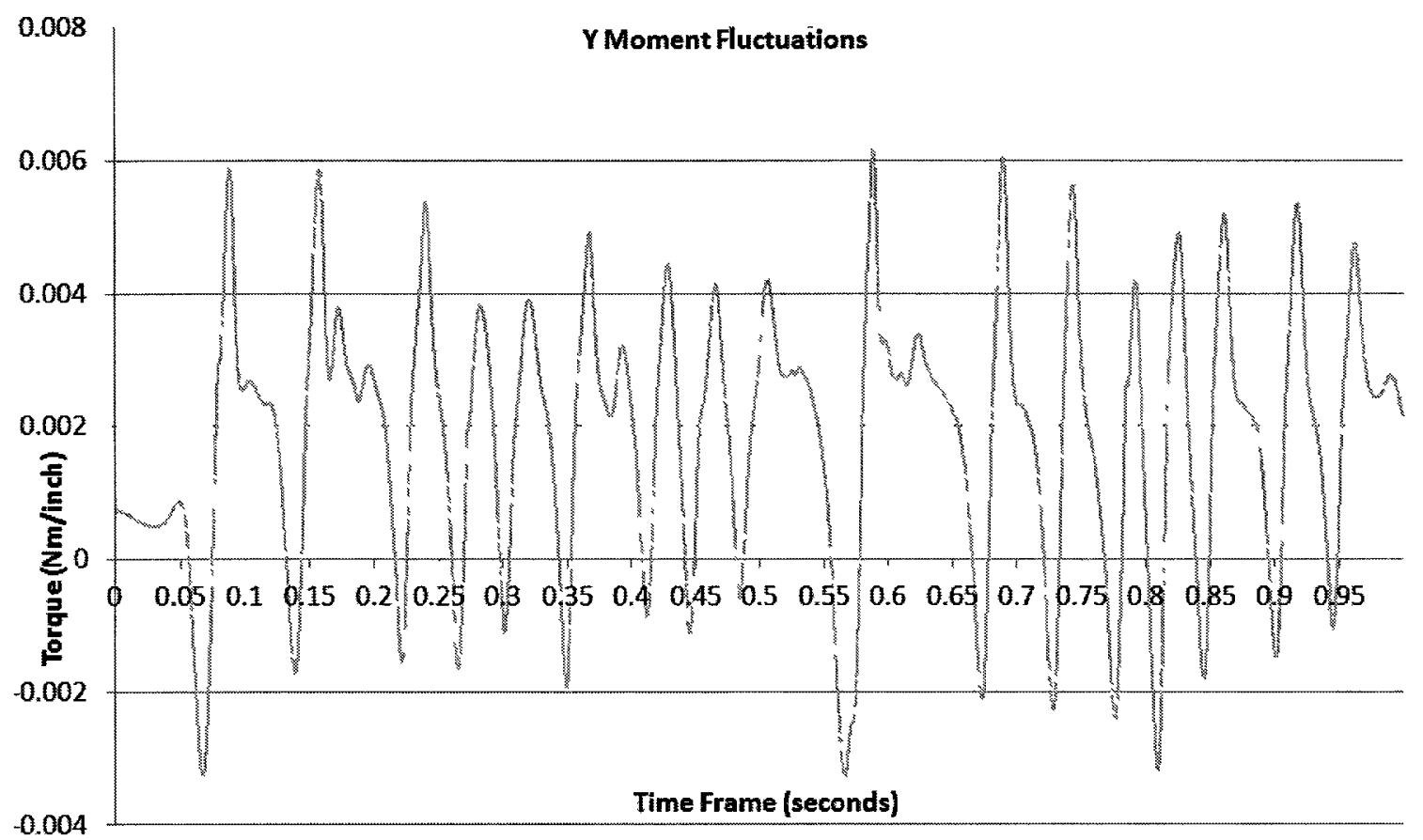

Figure 8.4: Panel torque over time

Since the lift and drag cause the torque along the $\mathrm{Y}$-axis, it makes sense that there are also 17 oscillations in the panel torque. Along with these results, the Courant number was also monitored.

\subsection{LES Frequency Analyses}

Measurements from the LES simulation were taken over 1 second. Data was acquired at a rate of $20 \mathrm{kHz}$. An example of $\mathrm{Fx}, \mathrm{Fz}$, and $\mathrm{My}$ at $40 \mathrm{~km} / \mathrm{hr}$ with a 60 degree angle panel using the LES setup previously described is shown in the time series below in Figure 8.5 . 




Figure 8.5: LES fluctuations

The spectral plot of these LES fluctuations is shown below in Figure 8.6. The vertical axis, representing the spectral power, is the amount a specific force or moment contributes across the spectrum.

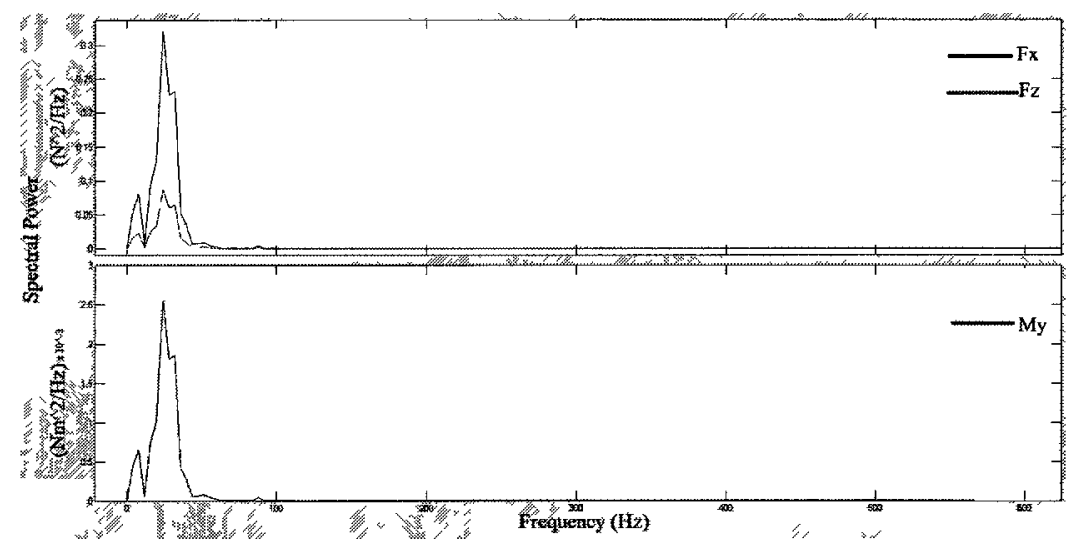

Figure 8.6: LES frequency spectrum

\subsubsection{Wind Tunnel Frequency Analyses}

The measurements taken were accumulated over 100 seconds. Data was acquired at a rate of $50 \mathrm{~Hz}$. This allowed the capture of force variations from the mean results. An example of $\mathrm{Fx}, \mathrm{Fz}$, and $\mathrm{My}$ at $40 \mathrm{~km} / \mathrm{hr}$ using the 60 degree angle panel at zero panel and building yaw, shown in the time series below in Figure 8.7 at position O. 


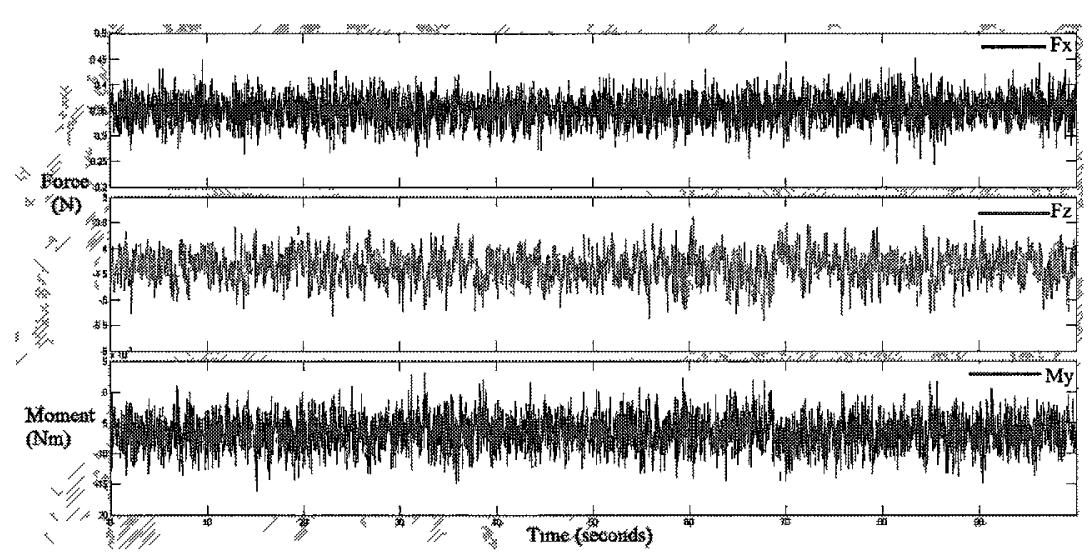

Figure 8.7: Wind tunnel fluctuations

The spectral plot of these wind tunnel fluctuations is shown below in Figure 8.8.

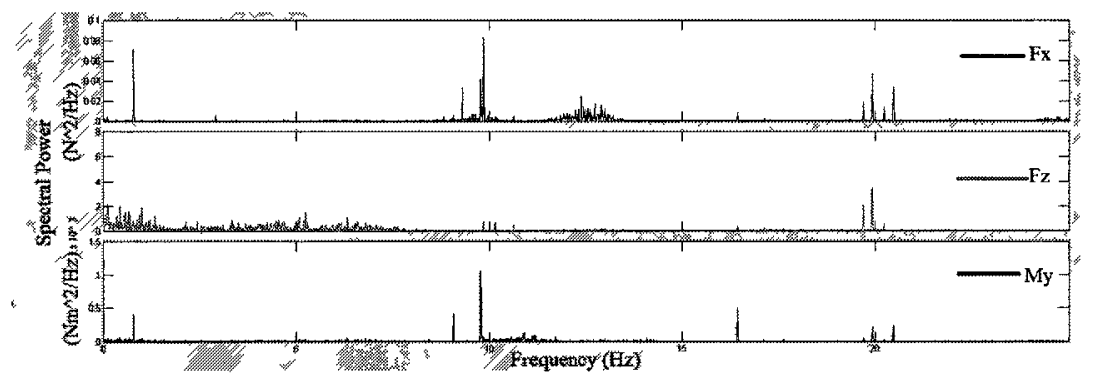

Figure 8.8: Wind tunnel frequency spectrum

\subsubsection{Comparison of LES to Wind Tunnel Data}

Two different methods exist in which to compare the data collected in the wind tunnel to that of the LES simulation. These are the magnitude of the forces and moments, and the frequencies of these forces and moments. Since the computer simulation was only performed in two dimensions, only $\mathrm{Fx}, \mathrm{Fz}$, and $\mathrm{My}$ can be compared.

As seen in Figure 6.3, the min and max Fx drag found in the LES computation was approximately $-0.06 \mathrm{~N} /$ in and $0.03 \mathrm{~N} /$ in. The measurements are in $\mathrm{N} /$ in because the domain thickness is one inch, effectively making the results two-dimensional. Since the panel width is known (3.69150 inches), multiplying the min and max Fx 
drag by the panel width effectively extrapolates the two-dimensional results to three dimensions, making the min and max drag for the entire panel $-0.22 \mathrm{~N}$ and $0.11 \mathrm{~N}$, respectively. In the wind tunnel model, the min and max Fx drag was approximately $0.25 \mathrm{~N}$ and $0.45 \mathrm{~N}$. Though there are differences, the values are the same order of magnitude, and discrepancies are likely due to described assumptions and errors. However, the frequency of oscillations may still be compared. From the computer simulation, it was previously stated that a frequency of $17 \mathrm{~Hz}$ was recorded. Looking at the wind tunnel Fx frequency spectrum in Figure 8.8, a peak in the spectrum occurs not only at approximately $17 \mathrm{~Hz}$, but also $10 \mathrm{~Hz}, 14 \mathrm{~Hz}$, and $20 \mathrm{~Hz}$. This does not however represent the frequency of vortex shedding off the solar panel, but represents the frequency of force oscillations from numerous sources. The 10 $\mathrm{Hz}$ matches the theoretical prediction for vortex shedding off the panel, and $14 \mathrm{~Hz}$ matches the frequency of shedding expected off the building's leading edge. The peak at $20 \mathrm{~Hz}$ is likely a harmonic multiple of the panel's fundamental tone at $10 \mathrm{~Hz}$, which can be present at the same time [21].

Besides errors sources, the source of discrepancies may be due to three dimensional effects in the wind tunnel that cannot be accounted for in the two dimensional computer simulation. The most obvious effect being that the actual solar panel width has end tips, which are not simulated in the computer. Just as an aircraft can develop wingtip vortexes, the solar panel may create similar fluctuating effects that are not shown in the LES computation, but in reality affect the solar panel model. The other possible cause could be edge effects from the side of the building. Being present in the wind tunnel, these effects are not simulated in the LES computation. Just as vortex shedding was observed off the leading edge of the building, similar vortexes could be shedding off the sides of the building and oscillating the wind flow in the Y-direction. Finally, the wind tunnel models a more accurate solar panel that included a post attached to the force plate. This means that forces measured represented the force 
the panel at its base on the building rooftop. The LES computation did not include this post due to the $2 \mathrm{D}$ nature of the simulation, and instead measured the forces acting directly on the surface of the panel. This would produce slightly different torque values since the moment arm of the post is not included, as well as the drag induced by the post in the wind tunnel.

The strut connecting the wind tunnel model to the force plate will contribute three quantities to the forces measured. First is the drag from the strut, second is the effect of the strut on the free air flow, and third is the models effect on the free air flow about the strut. The second and third items are called interference [14]. These properties are not measured in the computational models, and are thus not present in the result for the CFD simulations. 


\section{Chapter 9}

\section{Conclusions}

Successful research was performed to study the effect of wind over roof top solar panels. Using wind tunnel and CFD experiments, the flow characteristics of different solar panel configurations were obtained. It was found that the force and moment coefficients in the wind tunnel were insensitive to the Reynolds number. This allows for the coefficients to be used on full scale models to predict their expected loads.

After examining numerous roof top configurations in the wind tunnel, the following conclusions were made. For groups of panels placed together, the closer they are placed together, the less drag force and bending moment will be placed on the panels. Conversely, spacing panels further apart will decrease lift forces. Similarly, when locating just two panels, it is best to place them one behind the other to again reduce drag forces. The location of a panel on the roof surface produces the least drag when it is closer to the building's leading edge; however this also produces higher lift force. When implementing azimuth angles, it will be important to know that the lift and drag are highest when the panel faces away from the wind direction. Maintaining an elevation angle below 60 degrees will significantly reduce the forces and bending moments on the panel. In regards to the independent direction of the wind, low lift forces, and much higher drag force and bending moment will be experienced on the panel if the wind approaches the building from the direction of its corner. High 
velocity winds produce the most change in the drag force and bending moments.

Computer simulations revealed a vortex shedding frequency of $17 \mathrm{~Hz}$. Although this frequency is found in the wind tunnel data spectrum, the frequencies of $10 \mathrm{~Hz}, 20$ $\mathrm{Hz}$, and $14 \mathrm{~Hz}$ are much more prominent, and match the theoretical values expected for the panel's fundamental tone, first harmonic, and building's shedding frequency, respectively. The force and moment values from each experiment are of the approximate same magnitude, and although variations are present the reliability of the wind tunnel's measured frequency leads to more confidence in the wind tunnel values. The LES results, being based on a two dimensional model, likely did not represent the three dimensional wind tunnel experiment. However, assumptions such as panel rigidity, present in the computer simulation, may not be as confident in the wind tunnel.

Considerations to improve the experiment and decrease error include a more rigid wind tunnel model, and a more rigid connection of the panel post to force plate. More accurate markings and alignment methods would provide greater accuracy when aligning the building and panel models with the desired angles. A method for measuring the panel post height would also create a better confirmation of data.

The data trends in this research may be used as a basis for further work that may be used to justify equivalence with the National Building Code of Canada. Continuation of this work will benefit the solar industry by providing an inexpensive means to bring a solar panel product from the prototype phase to the public installation phase that satisfies the building code. For further study, it is recommended that the following section be considered. 


\subsection{Future Testing and Design Considerations}

Many more opportunities exist to examine certain design cases and scenarios. This section will highlight key areas that may be examined in the future in the event that this project is taken to the next level of development.

\subsubsection{Significance of Snow Accumulation}

Atmospheric wind loads are just one of the factors that can affect the integrity of a rooftop mounted solar panel. Another significant load is the effect from snow accumulation. The significance of snow load increases the further north a solar panel is installed. The wind tunnel at Carleton University has in the past been used for snow drift experiments, leading to the idea that this type of experiment can be repeated with the introduction of the building and its solar panel. The grains used to simulated the snow will be of critical importance in replicating the same snow conditions that would exist for a full scale model. The effect of the wind on snow drifting will be the benefit of accumulating snow in the wind tunnel. Being able to predict the amount and distribution of snow on the solar panel will also be better for structural designs, as well as better planning for panel exposure to the sun. Research in this area will be importance for to conformation with data from the National Building Code of Canada. Developing methods that trigger the panel under high stress (either from high winds or snow load) to collapse and lay flat, using possibly a spring mechanism, would be a valuable design to investigate.

\subsubsection{Significance of Model Variation}

This project utilized a specific model design for the solar panel. Although a unique design, it would be hoped that general characteristics of the model such as its general two dimensional panel shape, it's relatively smooth front surface and structure back 
surface, and positioning above the ground, would allow for a degree of relationship to exist between it and other solar panel designs of similar general characteristics. Such research that would establish these relationships would be the collecting of load data from various panel spans, chords, heights, and structural patterns. Any trends discovered could indeed reveal improved designs that would better withstand wind and snow loads. Furthermore, being able to relate the results of analysis on one panel could be more confidently interpolated into the design and manufacturing of a different panel.

\subsubsection{Panel Characteristics without Building}

During testing, the panel was continuously monitored while on the rooftop of the building. While this exposed the panel to its ideal environment, i.e. the atmospheric boundary layer moving over the building, the flow separation and vortex shedding of the building make it difficult to concentrate on the effect of the solar panel alone. Being able to test the solar panel without the building in a fully developed flow would allow a more complete understanding of the effects already tested in this project such as elevation angle, azimuth angle, and proximity to nearby panels. Also, while the current vision sees such solar panels existing on roof tops of large retail buildings, it is a fair assumption to say that investment may be made to have such solar panels as installations on the ground, even having large installations of many panels in a field. In such a case, testing the panel models without a building would be critical.

\subsubsection{Improve Model Assumptions}

As discussed earlier, it was assumed that the wind tunnel solar panel model was perfectly rigid. However, due to the material used in the model, this was not likely the case. Investigating new model materials, such as a type of metal (higher modulus 
of elasticity), or perhaps changing the geometry, would allow for this assumption to become more valid. A more rigid model would reduce possible aeroelastic effects, as well as reduce the effect of bending moments on the solar panel post. However, using a different material may require a new manufacturing process, since rapid prototyping is not possible with metal.

\subsubsection{Panel Modification Suggestions}

The testing process can also become a design loop, in which the results of tests can lead to the design of a panel that can better withstand wind and snow loads. Adding structural devices to the leading or trailing edge of the solar panel could reduce the lift or drag on the panel. This effect is seen on such devices installed on aircraft wings. Besides the desire to change the loads, being able to channel the airflow along the panel would allow the ability to remove snow off of the panel's surface. This eliminates the need to sweep the panels of snow accumulation.

\subsubsection{FEM stress analysis}

With the magnitude of the solar panel loads being known, it would be interesting to have an idea of how these loads affect the distribution of stress on the structure. Being able to plot trends in stress just as this project plotted trends in wind loads would provide unique information that could be used to design a more efficient solar panel with various reinforcements at areas known to have high stress, and as well as a reduction in material at areas of relatively lower stress. Being able to both design and test the panel structure repeatedly within one project would be a very efficient method of design rather than solely testing a design and giving the results to another project or company to design themselves. 


\subsubsection{Aeroelastic Effects}

In reality, perfect rigidity cannot exist for the full scale solar panel, and thus testing a non-rigid model would provide dynamic information to compliment the static information. Testing a flexible model would allow for the various modes of vibration that exist within the model, based on its mass distribution and shape, to be known. The mass and shape of the model would need to be properly related to the full scale model. These frequency modes could be verified with computational software, and could provide guidance on what the operating parameters of the solar panel are so that it may avoid aeroelastic fluctuations at key modal frequencies. Coupling FEM with CFD to model aeroelastic effects would be an ideal computational simulation. Identifying the stresses induced from fluttering, and the stability of the system along a spectrum of wind frequencies, would provide a complete understanding of the panel's operating conditions. 


\section{List of References}

[1] "Canadian action plan - ecoEnergy." http://www.ecoaction.gc.ca/ecoenergyecoenergie/index-eng.cfm.

[2] H. H. Mehrdad Shademan, "Wind loading on solar panels at different inclination angles," 2009.

[3] C. A. v. B. Chris P.W. Geurts, "Local wind loads on roof-mounted solar energy systems," 2007.

[4] C. C. on Building and F. Codes, "National Building Code of Canada Volume 1," tech. rep., National Research Council of Canada - Institute for Research Inconstruction, Ottawa, 2005.

[5] C. C. on Building and F. Codes, "National Building Code Structural Commentaries," tech. rep., National Research Council of Canada - Institute for Research Inconstruction, Ottawa, 2005.

[6] C. C. on Building and F. Codes, "National Building Code of Canada Volume 2," tech. rep., National Research Council of Canada - Institute for Research Inconstruction, Ottawa, 2005.

[7] E. S. D. U. ESDU70015, "Fluid forces and moments on flat plates," tech. rep., ESDU International Ltd, London, 1970.

[8] E. S. D. U. ESDU80003, "Mean forces and moments on rectangular prisms surface mounted structures in turbulence shear flow," tech. rep., ESDU International Ltd, London, 1979.

[9] Y.-C. F. Jerry M. Chen, "Strouhal numbers of inclined flat plates," Journal of Wind Engineering and Industrial Aerodynamics, 1996.

[10] C. KNISELY, "Stouhal numbers of rectangular cylinders at incidence: A review and new data," Journal of Fluids and Structures, 1989. 
[11] A. Davenport, "The relationship of wind structure to wind loading," tech. rep., University of Western Ontario, London, 1963.

[12] V. Karman, "The fundamentrals of statistical theory of turbulence," tech. rep., California Institute of Technology, California, 1933.

[13] A. Davenport, "Rationale for determining design wind velocities," tech. rep., University of Bistol, England, 1960.

[14] W. H. Rae and A. Pope, Low-Speed Wind Tunnel Testing. John Wiley and Sons, 1984.

[15] A. Davenport, "The dependence of wind loads on meteorological parameters," tech. rep., University of Western Ontario, London, 1967.

[16] H. Irwin, "The design of spires for wind simulation," tech. rep., National Research Council of Canada, Ottawa, 1980.

[17] S. McTavish, "Boundary layer wind tunnel spire, roughness, and turntable design." Carleton University, Ottawa, 2010.

[18] AMTI, HE6X6 User's Manual and Install Gunde. AMTI, 176 Waltham Street, Watertown, MA 02472 USA, version 2.0 ed., October 2005.

[19] AMTI, NetForce Acquısition Software Manual. 176 Waltham Street, Watertown, MA 02472 USA, 2.04.00 ed., December 2007.

[20] W. G. S. Hugh W. Coleman, Experımentatıon, Valıdatıon, and Uncertaınty Analysis for Engineers. Wiley, 2009.

[21] S. S. Rao, Mechanıcal Vıbratıons. Prentice Hall, 5th edition ed., 2010.

[22] J. L. Devore, Probabilnty and Statıstics for Engineering and the Sciences. Duxbury, 5th edition ed., 2000.

[23] E. Matida, "Aero4304 - lecture 10 notes," 2010.

[24] E. Matida, "Aero4304 - lecture 12 notes," 2010.

[25] H. Versteeg and W. Malalasekera, An ıntroductıon to Computatıonal Fluıd Dynamics: The Finute Volume Method. Prentice Hall, 1995.

[26] H. C. L. et al, "Flow around a cube in a turbulent boundry layer: LES and experiment," tech. rep., School of Engineering Sciences, University of Southhampton, UK, 2009. 


\section{Appendix A}

\section{The Taylor Series Method}

Considering the experimental result $F_{x}$ as an example, it is a function of the 12 data channels, the data reduction equation for $F_{x}$ takes the form of:

$$
\begin{aligned}
F_{x} & =(A x * S 11)+(A y * S 12)+(A z * S 13) \\
& +(B x * S 14)+(B y * S 15)+(B z * S 16) \\
& +(C x * S 17)+(C y * S 18)+(C z * S 19) \\
& +(D x * S 110)+(D y * S 111)+(D z * S 112)
\end{aligned}
$$

In this equation, the values S11 through S112 are representations of the calibration matrix supplied by the force plate manufacturer. The uncertainty of the result, $F_{x}$, is given by: 


$$
\begin{aligned}
U_{F_{x}}^{2} & =\left(\frac{\partial F_{x}}{\partial A x}\right)^{2} * U_{A x}^{2}+\left(\frac{\partial F_{x}}{\partial A y}\right)^{2} * U_{A y}^{2}+\left(\frac{\partial F_{x}}{\partial A z}\right)^{2} * U_{A z}^{2} \\
& +\left(\frac{\partial F_{x}}{\partial B x}\right)^{2} * U_{B x}^{2}+\left(\frac{\partial F_{x}}{\partial B y}\right)^{2} * U_{B y}^{2}+\left(\frac{\partial F_{x}}{\partial B z}\right)^{2} * U_{B z}^{2} \\
& +\left(\frac{\partial F_{x}}{\partial C x}\right)^{2} * U_{C x}^{2}+\left(\frac{\partial F_{x}}{\partial C y}\right)^{2} * U_{C y}^{2}+\left(\frac{\partial F_{x}}{\partial C z}\right)^{2} * U_{C z}^{2} \\
& +\left(\frac{\partial F_{x}}{\partial D x}\right)^{2} * U_{D x}^{2}+\left(\frac{\partial F_{x}}{\partial D y}\right)^{2} * U_{D y}^{2}+\left(\frac{\partial F_{x}}{\partial D z}\right)^{2} * U_{D z}^{2}
\end{aligned}
$$

where $U_{A x}, U_{A y} \ldots$ etc are the uncertainties in the measured variables $\mathrm{Ax}, \mathrm{Ay}, \mathrm{Az}$, $\mathrm{Bx}$, etc, called the absolute uncertainties [20]. These uncertainties are expressed with the same level of confidence, assumed to be 95

$$
\begin{aligned}
\left(\frac{U_{F_{x}}}{F_{x}}\right)^{2} & =\left(\frac{A x}{F_{x}} \frac{\partial F_{x}}{\partial A x}\right)^{2} * \frac{U_{A x}{ }^{2}}{A x}+\left(\frac{A y}{F_{x}} \frac{\partial F_{x}}{\partial A y}\right)^{2} * \frac{U_{A y}{ }^{2}}{A y}+\left(\frac{A z}{F_{x}} \frac{\partial F_{x}}{\partial A z}\right)^{2} * \frac{U_{A z}{ }^{2}}{A z} \\
& +\left(\frac{B x}{F_{x}} \frac{\partial F_{x}}{\partial B x}\right)^{2} * \frac{U_{B x}{ }^{2}}{B x}+\left(\frac{B y}{F_{x}} \frac{\partial F_{x}}{\partial B y}\right)^{2} * \frac{U_{B y}{ }^{2}}{B y}+\left(\frac{B z}{F_{x}} \frac{\partial F_{x}}{\partial B z}\right)^{2} * \frac{U_{B z}{ }^{2}}{B z} \\
& +\left(\frac{C x}{F_{x}} \frac{\partial F_{x}}{\partial C x}\right)^{2} * \frac{U_{C x}{ }^{2}}{C x}+\left(\frac{C y}{F_{x}} \frac{\partial F_{x}}{\partial C y}\right)^{2} * \frac{U_{C y}{ }^{2}}{C y}+\left(\frac{C z}{F_{x}} \frac{\partial F_{x}}{\partial C}\right)^{2} * \frac{U_{C z}{ }^{2}}{C z} \\
& +\left(\frac{D x}{F_{x}} \frac{\partial F_{x}}{\partial D x}\right)^{2} * \frac{U_{D x}{ }^{2}}{D x}+\left(\frac{D y}{F_{x}} \frac{\partial F_{x}}{\partial D y}\right)^{2} * \frac{U_{D y}{ }^{2}}{D y}+\left(\frac{D z}{F_{x}} \frac{\partial F_{x}}{\partial D z}\right)^{2} * \frac{U_{D z}{ }^{2}}{D z}
\end{aligned}
$$

where $U_{F_{x}} / F_{x}$ is the relative uncertainty of the result $F_{x}$. Factors like $U_{A x} / A x$ are the relative uncertainties for each variable. The factors in parentheses that multiply the relative uncertainties of the variables are called uncertainty magnification factors (UMFs) [20], defined as the example below:

$$
\begin{aligned}
U M F_{A x} & =\frac{A x}{F_{x}} \frac{\partial F_{x}}{\partial A x}=S 11 \frac{A x}{F_{x}} \\
U M F_{A y} & =\frac{A y}{F_{x}} \frac{\partial F_{x}}{\partial A y}=S 12 \frac{A y}{F_{x}}
\end{aligned}
$$




$$
\begin{aligned}
U M F_{A z} & =\frac{A z}{F_{x}} \frac{\partial F_{x}}{\partial A z}=S 13 \frac{A z}{F_{x}} \\
U M F_{B x} & =\frac{B x}{F_{x}} \frac{\partial F_{x}}{\partial B x}=S 14 \frac{B x}{F_{x}} \\
U M F_{B y} & =\frac{B y}{F_{x}} \frac{\partial F_{x}}{\partial B y}=S 15 \frac{B y}{F_{x}} \\
U M F_{B z} & =\frac{B z}{F_{x}} \frac{\partial F_{x}}{\partial B z}=S 16 \frac{B z}{F_{x}} \\
U M F_{C x} & =\frac{C x}{F_{x}} \frac{\partial F_{x}}{\partial C x}=S 17 \frac{C x}{F_{x}} \\
U M F_{C y} & =\frac{C y}{F_{x}} \frac{\partial F_{x}}{\partial C y}=S 18 \frac{C y}{F_{x}} \\
U M F_{C z} & =\frac{C z}{F_{x}} \frac{\partial F_{x}}{\partial C z}=S 19 \frac{C z}{F_{x}} \\
U M F_{D x}= & \frac{D x}{F_{x}} \frac{\partial F_{x}}{\partial D x}=S 110 \frac{D x}{F_{x}} \\
U M F_{D y}= & \frac{D y}{F_{x}} \frac{\partial F_{x}}{\partial D y}=S 111 \frac{D y}{F_{x}} \frac{\partial F_{x}}{\partial D z}=S 112 \frac{D z}{F_{x}} \\
U F_{D z} &
\end{aligned}
$$

Substituting the UMFs into the relative uncertainty equation for the result $F_{x}$ yields:

$$
\begin{aligned}
\left(\frac{U_{F_{x}}}{F_{x}}\right)^{2} & =\left(S 11 \frac{A x}{F_{x}}\right)^{2} *\left(\frac{U_{A x}}{A x}\right)^{2}+\left(S 12 \frac{A y}{F_{x}}\right)^{2} *\left(\frac{U_{A y}}{A y}\right)^{2}+\left(S 13 \frac{A z}{F_{x}}\right)^{2} *\left(\frac{U_{A z}}{A z}\right)^{2} \\
& +\left(S 14 \frac{B x}{F_{x}}\right)^{2} *\left(\frac{U_{B x}}{B x}\right)^{2}+\left(S 15 \frac{B y}{F_{x}}\right)^{2} *\left(\frac{U_{B y}}{B y}\right)^{2}+\left(S 16 \frac{B z}{F_{x}}\right)^{2} *\left(\frac{U_{B z}}{B z}\right)^{2} \\
& +\left(S 17 \frac{C x}{F_{x}}\right)^{2} *\left(\frac{U_{C x}}{C x}\right)^{2}+\left(S 18 \frac{C y}{F_{x}}\right)^{2} *\left(\frac{U_{C y}}{C y}\right)^{2}+\left(S 19 \frac{C z}{F_{x}}\right)^{2} *\left(\frac{U_{C z}}{C z}\right)^{2} \\
& +\left(S 110 \frac{D x}{F_{x}}\right)^{2} *\left(\frac{U_{D x}}{D x}\right)^{2}+\left(S 111 \frac{D y}{F_{x}}\right)^{2} *\left(\frac{U_{D y}}{D y}\right)^{2}+\left(S 112 \frac{D z}{F_{x}}\right)^{2} *\left(\frac{U_{D z}}{D z}\right)^{2}
\end{aligned}
$$


For the purpose of this analysis, it will be assumed that the measured variables are of the same value, i.e. $A x=A y=A z=B x=\ldots$. This reduces the above equation to the much simpler form below:

$$
\left(\frac{U_{F_{x}}}{F_{x}}\right)_{\text {relativeuncertainty }}=\frac{U_{A x}}{F_{x}} \sqrt{\left(S 11^{2}+S 12^{2}+\ldots\right)}
$$




\section{Appendix B}

\section{Wind Tunnel Results Matrix}

This appendix will present a tabulated representation of the entire dataset collected in the wind tunnel. The data code in the leftmost column that represents each data set is explained in the following diagram:

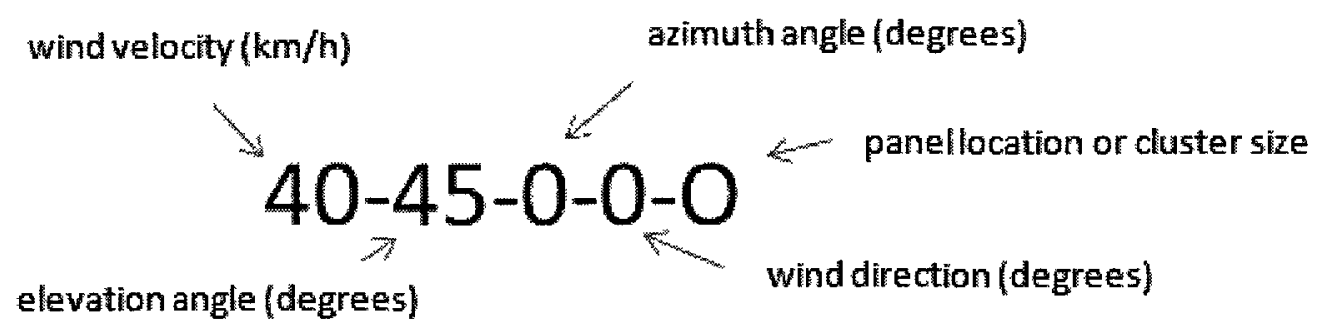

For each trial run, sampling was performed at $50 \mathrm{~Hz}$ over 100 seconds, creating a time series of 5000 samples. Since temperature, pressure, and the physical setup remained the same, only variations in the force existed. These values are averaged together to provide a single data point. The standard deviation from this mean is found using the following equation:

$$
S=\sqrt{\frac{1}{N-1} \sum_{i=1}^{N}\left(x_{i}-\bar{x}\right)^{2}}
$$

where $S$ is the standard deviation of the sample observed from the mean, $N$ is 
the amount of samples, $x$ is the value of the sample items, and $\bar{x}$ is the mean value of the sample observed [22]. For this experiment, $N$ was 5000 , while the mean value and sample item value varied for each experiment trial.

The wind tunnel data set and the sample standard deviations are presented in the follwing tables, first with a table for force values, and then for moments.

\begin{tabular}{|c|c|c|c|c|c|c|}
\hline Data Code & Fx(N) & Fy $(N)$ & $F_{2}(\mathrm{M})$ & S.FX(N) & S.F $(N)$ & S_F (N) \\
\hline $15-45-0-0-0$ & 0.0073 & 0.0018 & -0.0031 & 0.0483 & 0.0069 & 0.0303 \\
\hline $20-45-0-0-0$ & 0.0155 & 0.0010 & -0.0021 & 0.0164 & 0.0074 & 0,0310 \\
\hline $25-45-0-0-0$ & 0.0269 & 0.0003 & -0.0008 & 0.0185 & 0.0082 & 0.0227 \\
\hline $30-45-0-0-0$ & 0.0389 & 0.0009 & 0.0018 & 0.0319 & 0.0126 & 0.0393 \\
\hline $35-45-0-0-0$ & 0.0528 & -0.0015 & 0.0030 & 0.0417 & 0.0176 & 0.0390 \\
\hline $40-15-0-0-0$ & 0.0245 & -0.0123 & -0.0203 & 0.0233 & 0.0136 & 0.0476 \\
\hline $40-30-0-0-0$ & 0.0532 & -0.0085 & 0.0129 & 0.0358 & 0.0175 & 0.0471 \\
\hline $40-45-0-0-\mathrm{CLG}$ & 0.0315 & -0.0033 & -0.0163 & 0.0397 & 0.0192 & 0.0333 \\
\hline $40-45-0-0-\operatorname{csm}$ & 0.0241 & -0.0016 & -0.0140 & 0.0448 & 0.0215 & 0.0366 \\
\hline $40-45-0-0-\operatorname{CSP}$ & 0.0485 & -0.0028 & -0.0021 & 0.0472 & 0.0225 & 0.0381 \\
\hline $40-45-0-0-D E$ & 0.0634 & 0.0195 & 0.0001 & 0.0501 & 0.0234 & 0.0402 \\
\hline $40-45-0-0-D^{2}$ & 0.0611 & 0.0201 & -0.0010 & 0.0496 & 0.0225 & 0.0395 \\
\hline $40-45-0-0-0 N$ & 0.0359 & 0.0022 & -0.0225 & 0.0462 & 0.0195 & 0.0366 \\
\hline $40-45-0-0-\mathrm{DN}^{2}$ & 0.0547 & 0.0086 & -0.0101 & 0.0503 & 0.0222 & 0.0391 \\
\hline $40-45-0-0-D S$ & 0.0572 & 0.0140 & -0.0062 & 0.0478 & 0.0219 & 0.0372 \\
\hline $40-45-0-0-0 S^{3}$ & 0.0573 & 0.0154 & -0.0043 & 0.0504 & 0.0229 & 0.0379 \\
\hline $40-45-0-0-N$ & 0.0502 & 0.0018 & -0.0056 & 0.0567 & 0.0241 & 0.0428 \\
\hline $40-45-0-0-\mathrm{N}^{\prime}$ & 0.0356 & -0.0023 & -0.0102 & 0.0603 & 0.0224 & 0.0457 \\
\hline $40-45-0-0-0$ & 0.0688 & -0.0024 & 0.0050 & 0.0516 & 0.0217 & 0,0379 \\
\hline $40-45-0-0-5$ & 0.0634 & -0.0008 & 0.0055 & 0.0465 & 0.0224 & 0.0386 \\
\hline $40-45-0-0-5^{2}$ & 0.0565 & -0.0018 & 0.0020 & 0.0379 & 0.0190 & 0.0325 \\
\hline $40-45-0-0-W$ & 0.0540 & -0.0005 & 0.0028 & 0.1138 & 0.1021 & 0.0636 \\
\hline $40-45-0-0-W^{\prime}$ & 0.0552 & 0.0026 & -0.0011 & 0.0848 & 0.1003 & 0.0535 \\
\hline $40-45-0-15-0$ & 0.0661 & -0.0030 & 0.0077 & 0.0500 & 0.0221 & 0.0393 \\
\hline $40-45-0-30-0$ & 0.0860 & -0.0026 & 0.0260 & 0.0459 & 0.0211 & 0.0379 \\
\hline $40-45-0-45-0$ & 0.1037 & -0.0001 & 0.0346 & 0.0366 & 0.0181 & 0.0324 \\
\hline $40-45-120-0-0$ & 0.0412 & 0.0515 & -0.0933 & 0.0440 & 0.0439 & 0.0450 \\
\hline $40-45-150-0-0$ & 0.0819 & 0.0444 & -0.1191 & 0.0594 & 0.0353 & 0.0538 \\
\hline $40-45-180-0-0$ & 0.0864 & -0.0077 & -0.1180 & 0.0648 & 0.0215 & 0.0554 \\
\hline $40-45-30-0-0$ & 0.0555 & -0.0369 & -0.0027 & 0.0377 & 0.0332 & 0.0368 \\
\hline $40-45-60-0-0$ & 0.0180 & -0.0311 & -0.0228 & 0.0232 & 0.0360 & 0.0346 \\
\hline $40-45-90-0-0$ & 0.0047 & 0.0083 & -0.0541 & 0.0246 & 0.0311 & 0.0338 \\
\hline $40-60-0-0-0$ & 0.4126 & -0.0259 & -1.6948 & 0.0758 & 0.3651 & 0.1556 \\
\hline $45-45-0-0-0$ & 0.0862 & -0.0037 & 0.0063 & 0.0692 & 0.0267 & 0.0460 \\
\hline
\end{tabular}




\begin{tabular}{|c|c|c|c|c|c|c|}
\hline Data Code & $\mathrm{Mx}(\mathrm{Nm})$ & $\mathrm{My}(\mathrm{Nm})$ & $\mathrm{Mz}(\mathrm{Nm})$ & $S_{\mathrm{M}} \times(\mathrm{Nm})$ & $5 \mathrm{My}(\mathrm{Nm})$ & $\mathrm{S}^{\prime} \mathrm{Mz}(\mathrm{Nm})$ \\
\hline $15-45-0-0-0$ & -0.0029 & -0.0027 & -0.0042 & 0.0007 & 0.0004 & 0.0005 \\
\hline $20-45-0-0-0$ & -0.0028 & -0.0032 & -0.0044 & 0.0006 & 0.0007 & 0.0007 \\
\hline $25-45-0-0-0$ & -0.0027 & -0.0037 & -0.0046 & 0.0007 & 0.0007 & $0.000 \%$ \\
\hline $30-45-0-0-0$ & -0.0025 & -0.0044 & -0.0046 & 0.0012 & 0.0011 & 0.0010 \\
\hline $35-45-0-0-0$ & -0.0024 & -0.0051 & -0.0049 & 0.0016 & 0.0016 & 0.0014 \\
\hline $40-15-0-0-0$ & .0 .0029 & -0.0035 & -0.0042 & 0.0027 & 0.0023 & 0.0011 \\
\hline $40-30-0-0-0$ & -0.0024 & -0.0045 & -0.0035 & 0.0023 & 0.0020 & 0.0015 \\
\hline $40-45-0-0-C L G$ & -0.0026 & -0.0043 & -0.0045 & 0.0019 & 0.0017 & 0.0016 \\
\hline $40-45-0-0-C 5 M$ & -0.0025 & -0.0042 & -0.0045 & 0.0023 & 00021 & 0.0018 \\
\hline $40-45-0-0-\operatorname{CSP}$ & -0.0023 & -0.0050 & -0.0044 & 0.0021 & 0.0021 & 0.0019 \\
\hline 40-45-0-0-DE & -0.0020 & -0.0053 & -0.0044 & 0.0021 & 0.0022 & 0.0019 \\
\hline $40-45-0-0-\mathrm{DE}^{i}$ & -0.0020 & -0.0053 & -0.0043 & 0.0022 & 0.0022 & 0.0020 \\
\hline $40-45-0-0-0 N$ & -0.0025 & -0.0045 & -0.0045 & 0.0025 & 0.0022 & 0.0018 \\
\hline $40-45-0-0-D^{\prime}$ & -0.0022 & -0.0051 & -0.0047 & 0.0023 & 0.0022 & 0.0019 \\
\hline $40-45-0-0-05$ & -0.0021 & -0.0052 & -0.0047 & 0.0021 & 0.0021 & 0.0019 \\
\hline $40-45-0-0-05^{4}$ & -0.0021 & -0.0052 & -0.0045 & 0.0021 & 0.0021 & 0.0019 \\
\hline $40-45-0-0-\mathrm{N}$ & -0.0022 & -0.0050 & -0.0040 & 0.0024 & 0.0025 & 0.0020 \\
\hline $40-45-0-0-\mathrm{N}^{\prime}$ & -0.0026 & -0.0045 & -0.0042 & 0.0034 & 0.0032 & 0.0022 \\
\hline $40-45-0-0-0$ & -0.0022 & -0.0059 & -0.0049 & 0.0022 & 0.0022 & 0.0018 \\
\hline $40-45-0-0-5$ & -0.0017 & -0.0057 & -0.0038 & 0.0020 & 0.0020 & 0.0019 \\
\hline $40-45-0-0-5^{t}$ & .0 .0022 & -0.0052 & -0.0046 & 0.0016 & 0.0017 & 0.0016 \\
\hline $40-45-0-0-W$ & -0.0032 & -0.0058 & -0.0034 & 0.0045 & 0.0049 & 0.0023 \\
\hline $40-45-0-0-W^{t}$ & -0.0027 & -0.0059 & -0.0043 & 0.0035 & 0.0041 & 0.0019 \\
\hline $40-45-0-15-0$ & -0.0021 & -0.0056 & -0.0040 & 0.0021 & 0.0022 & 0.0018 \\
\hline $40-45-0-30-0$ & -0.0017 & -0.0068 & -0.005 & 0.0019 & 0.0020 & 0.0018 \\
\hline $40-45-0-45-0$ & -0.0013 & -0.0074 & -0.0048 & 0.0015 & 0.0016 & 0.0015 \\
\hline $40-45-120-0-0$ & -0.0005 & -0.0056 & -0.0047 & 0.0026 & 0.0027 & 0.0016 \\
\hline $40-45-150-0-0$ & -0.0009 & 0.0076 & -0.0040 & 0.0028 & 0.0032 & 0.0020 \\
\hline $40-45-180-0-0$ & -0.0026 & -0.0078 & -0.0044 & 0.0020 & 0.0029 & 0.0020 \\
\hline $40-45-30-0-0$ & -0.0044 & -0.0047 & -0.0041 & 0.0021 & 0.0018 & 0.0013 \\
\hline $40-45-60-0-0$ & -0.0045 & -0.0032 & -0.0034 & 0.0020 & 0.0019 & 0.0013 \\
\hline $40-45-90-0-0$ & -0.0027 & -0.0034 & -0.0040 & 0.0018 & 0.0019 & 0.0012 \\
\hline $40-60-0-0-0$ & 0.0102 & -0.0417 & -0.0010 & 0.0148 & 0.0057 & 0.0078 \\
\hline $45-45-0-0-0$ & -0.0020 & -0.0068 & -0.0050 & 0.0029 & 0.0029 & 0.0024 \\
\hline
\end{tabular}




\section{Appendix C}

\section{Non-dimensionalized Results}

To allow for more direct comparison with similar model experiments, the results need to be non-dimensionalized. This removes the physical units from the results, creating a coefficient that may be used on another panel of different scale or wind conditions. Only using this method may drag coefficients be compared. Using different approaches to achieve force and moment coefficients will make them incomparable. The force coefficients for are found in the following manner:

$$
C_{F(x, y, z)}=\frac{F(x, y, z)}{0.5 \rho V^{2} A}
$$

where $\rho$ is the density of air, $\mathrm{V}$ is the wind velocity in front of the panel in the streamwise direction, and $\mathrm{A}$ is the projected frontal area of the panel model. The projected area is constantly changing as the panel changes its elevation angle, and its azimuth angle. The surfaces that must be projected are the panel stop surface, its front edge, the side edge, and the support post. The following equation is used to calculate the projected area: 


$$
A=C \sin \alpha W \cos \phi+T \cos \alpha W \cos \phi+C T \sin \phi+D(L-0.5 C \sin \alpha)
$$

where $\mathrm{C}$ is the panel chord, $\alpha$ is the panel elevation angle, $\mathrm{T}$ is the panel thickness, $\mathrm{W}$ is the panel width, $\phi$ is the panel azimuth angle, $\mathrm{D}$ is the support post diameter, and $L$ is the support post length. Substituting this equation along with the proper wind velocity into the equation for $C_{F(x, y, z)}$ allows for the force coefficient to be attained. For the moment coefficients, a similar manner is following, using the following equation:

$$
C_{M(x, y, z)}=\frac{M(x, y, z)}{0.5 \rho V^{2} A C}
$$

where now the panel chord, C, is introduced as a characteristic length to eliminate the additional unit present in the moments compared to the previous forces. Using these equations for the force and moment coefficients, the forces and moments tabulated in the previous appendix are now listed again in their non-dimensionalized forms in the following table: 


\begin{tabular}{|c|c|c|c|c|c|c|}
\hline Data Code & CFx & CFy & CFz & CMx & CMY & $\mathrm{CMz}$ \\
\hline $15-45-0-0-0$ & 0.76 & 5.51 & 4.43 & 9.13 & 3.23 & 6.11 \\
\hline $20-45-0-0-0$ & 0.90 & 1.67 & 1.68 & 5.02 & 2.14 & 3.58 \\
\hline $25-45-0-0-0$ & 1.00 & 0.36 & 0.42 & 3.11 & 1.61 & 2.39 \\
\hline $30-45-0-0-0$ & 1.00 & 0.69 & 0.65 & 2.03 & 1.32 & 1.68 \\
\hline $35-45-0-0-0$ & 1.00 & 0.80 & 0.78 & 1.42 & 1.12 & 1.30 \\
\hline $40-15-0-0-0$ & 0.87 & 12.71 & 9.83 & 3.15 & 1.44 & 2.10 \\
\hline $40-30-0-0-0$ & 1.06 & 4.92 & 3.53 & 1.48 & 1.05 & 0.98 \\
\hline $40-45-0-0-C L G$ & 0.46 & 1.41 & 3.24 & 1.19 & 0.73 & 0.92 \\
\hline $40-45-0-0-C S M$ & 0.35 & 0.67 & 2.79 & 1.10 & 0.72 & 0.92 \\
\hline $40-45-0-0-C S P$ & 0.70 & 1.18 & 0.41 & 1.03 & 0.86 & 0.90 \\
\hline $40-45-0-0-D E$ & 0.92 & 8.25 & 0.02 & 0.89 & 0.90 & 0.89 \\
\hline $40-45-0-0-\mathrm{DE}^{\prime}$ & 0.89 & 8.48 & 0.21 & 0.90 & 0.90 & 0.88 \\
\hline $40-45-0-0-D N$ & 0.52 & 0.95 & 4.48 & 1.14 & 0.77 & 0.92 \\
\hline $40-45-0-0-D^{\prime}$ & 0.79 & 3.64 & 2.00 & 0.97 & 0.87 & 0.96 \\
\hline $40-45-0-0-D S$ & 0.83 & 5.93 & 1.24 & 0.95 & 0.88 & 0.95 \\
\hline $40-45-0-0-\mathrm{DS}^{4}$ & 0.83 & 6.51 & 0.85 & 0.93 & 0.88 & 0.92 \\
\hline $40-45-0-0-N$ & 0.73 & 0.75 & 1.11 & 0.98 & 0.85 & 0.82 \\
\hline $40-45-0-0-\mathrm{N}^{\prime}$ & 0.52 & 0.97 & 2.02 & 1.16 & 0.76 & 0.86 \\
\hline $40-45-0-0-0$ & 1.00 & 1.00 & 1.00 & 1.00 & 1.00 & 1.00 \\
\hline $40-45-0-0-5$ & 0.92 & 0.36 & 1.09 & 0.78 & 0.96 & 0.77 \\
\hline $40-45-0-0-S^{\prime}$ & 0.82 & 0.77 & 0.40 & 0.99 & 0.88 & 0.93 \\
\hline $40-45-0-0-W$ & 0.78 & 0.23 & 0.55 & 1.45 & 0.98 & 0.70 \\
\hline $40-45-0-0-W^{\prime}$ & 0.80 & 1.08 & 0.22 & 1.21 & 1.01 & 0.88 \\
\hline $40-45-0-15-0$ & 0.96 & 1.26 & 1.52 & 0.95 & 0.94 & 0.81 \\
\hline $40-45-0-30-0$ & 1.25 & 1.11 & 5.16 & 0.74 & 1.15 & 1.05 \\
\hline $40-45-0-45-0$ & 1.51 & 0.05 & 6.87 & 0.60 & 1.25 & 0.97 \\
\hline $40-45-120-0-0$ & 1.16 & 42.30 & 35.95 & 0.29 & 1.35 & 1.35 \\
\hline $40-45-150-0-0$ & 1.36 & 21.49 & 27.05 & 0.40 & 1.31 & 0.83 \\
\hline $40-45-180-0-0$ & 1.25 & 3.27 & 23.43 & 1.17 & 1.33 & 0.90 \\
\hline $40-45-30-0-0$ & 0.92 & 17.87 & 0.62 & 2.01 & 0.81 & 0.84 \\
\hline $40-45-60-0-0$ & 0.51 & 25.52 & 8.80 & 2.87 & 0.77 & 0.97 \\
\hline $40-45-90-0-0$ & 3.37 & 175.49 & 534.54 & 4.89 & 2.33 & 3.33 \\
\hline $40-60-0-0-0$ & 4.97 & 9.09 & 279.23 & 3.80 & 5.86 & 0.18 \\
\hline $45-45-0-0-0$ & 0.99 & 1.23 & 0.99 & 0.71 & 0.91 & 0.81 \\
\hline
\end{tabular}




\section{Appendix D}

\section{Computational Fluid Dynamics}

\section{Background}

\section{D.1 Modelling Turbulence using RANS and LES}

Reynolds averaged Navier-Stokes (RANS) is a method of modelling turbulence by taking the Navier-Stokes equation:

$$
\frac{\partial U_{\imath}}{\partial t}+U_{\jmath} \frac{\partial U_{\imath}}{\partial x_{\jmath}}=-\frac{1}{\rho} \frac{\partial P}{\partial x_{\imath}}+\frac{\mu}{\rho} \frac{\partial^{2} U_{\imath}}{\partial x_{\jmath} \partial x_{\jmath}}
$$

and substituting for velocity the average velocity with the addition of a velocity component fluctuating from the average, shown as $U_{\imath}=\bar{U}_{\imath}+u_{\imath}$ then averaging the Navier Stokes equation becomes:

$$
\frac{\partial \bar{U}_{\imath}}{\partial t}+\bar{U}_{\jmath} \frac{\partial \bar{U}_{\imath}}{\partial x_{\jmath}}=-\frac{1}{\rho} \frac{\partial \rho P}{\partial x_{\imath}}+\frac{1}{\rho} \frac{\partial}{\partial x_{\jmath}}\left(\mu \frac{\partial \bar{U}_{\imath}}{\partial x_{\jmath}}-\rho u_{\imath} u_{\jmath}\right)
$$

where $\bar{U}_{\imath} \equiv \frac{1}{t_{1}-t_{0}} \int_{t_{0}}^{t_{1}} U_{\imath}(t) d t$. [23]

RANS, however, does not capture turbulent structures such as eddies and vortex 
shedding. To capture these unsteady structures, more computationally expensive methods may be used.

Large eddy simulation (LES) approximates small scale turbulent structures while simulating the large structures. LES is similar to using RANS on small scale turbulence, and Direct Numerical Simulation on the large structures. Using filtering, the total field containing only large scale components of the velocity field must be initially defined. This large scale field is the local average of the complete field. The filtered Navier-Stokes equations used are:

$$
\begin{gathered}
\frac{\partial \bar{u}}{\partial x_{\imath}}=0 \\
\frac{\partial \bar{u}}{\partial t}+\frac{\partial}{\partial x_{\jmath}}\left(\bar{u}_{\imath} \bar{u}_{\jmath}\right)=-\frac{1}{\rho} \frac{\partial \bar{p}}{\partial x_{\imath}}-\frac{\partial \tau_{\imath \jmath}}{\partial x_{\jmath}}+\mu \frac{\partial^{2} \bar{u}_{\imath}}{\partial x_{\jmath} \partial x_{\jmath}}
\end{gathered}
$$

where $\tau_{\imath \jmath}=u_{\imath} \bar{u}_{\jmath}-\bar{u}_{\imath} \bar{u}_{\jmath}$

By using the eddy viscosity model, the stresses become $\tau_{\imath \jmath}-\frac{\delta_{\imath 2}}{3} \tau_{k k}=-2 \mu_{T} \bar{S}_{\imath \jmath}$, where $\mu_{T} \bar{S}$ is the large scale strain rate tensor. The eddy viscosity, $\mu_{T}$, is written as $\mu_{T}=\left(C_{s} \bar{\triangle}\right)^{2}|\bar{S}|$, where $C_{s}$ is the Smagorinsky constant used in this model. [24]

\section{D.2 Finite Volume Method}

A numerical method is needed to model and solve the Navier-Stokes equations. The finite volume method can be implemented by following three main steps:

Step 1: Grid generation: The domain is divided into discrete control volumes. Nodal points are placed in between the boundaries of the control volumes. It is common practice to align the physical boundaries with that of a control volume boundary. Nodal points are identified by a $\mathrm{P}$, with its neighbours on the left and 
right in a one-dimensional geometry identified by $\mathrm{W}$ and $\mathrm{E}$ respectively. West sides of a control volume are designated by a w, and the east side by an e. The distances between nodes $\mathrm{W}$ and $\mathrm{P}$ and nodes $\mathrm{P}$ and $\mathrm{E}$ are respectively identified as $\delta x_{W P}$ and $\delta x_{P E}$. As such the space between the face $\mathrm{w}$ and point $\mathrm{P}$ and between $\mathrm{P}$ and face $\mathrm{e}$ are respectively $\delta x_{w P}$ and $\delta x_{P e}$. The width of the control volume is $\delta=\delta x_{w e} \cdot[25]$

Step 2: Discretization: The integration of the governing equations over a control volume yields a discretized equation at its nodal point P. A diffusive flux of $\phi$ exiting the east face minus the diffusive flux of $\phi$ entering the west face equals the generation of $\phi$. The interface diffusion coefficient $\Gamma$ and the gradient $d \phi / d x$ at east and west are required to derive useful forms of the discretized equations. An approximate distribution of properties between nodal points is used, called central differencing. [25]

In addition to east and west neighbours a two dimensional grid node $\mathrm{P}$ will also have north, $\mathrm{N}$, and south, $\mathrm{S}$ neighbours. The general discretized equation for the interior nodes becomes:

$$
a_{P} \phi_{P}=a_{W} \phi_{W}+a_{E} \phi_{E}++a_{N} \phi_{N}+a_{S} \phi_{S}+S_{u}
$$

where:

\begin{tabular}{|l|l|l|l|l|}
\hline$a_{W}$ & $a_{E}$ & $a_{S}$ & $a_{N}$ & $a_{P}$ \\
\hline$\frac{\Gamma_{w} A_{w}}{\delta x_{W P}}$ & $\frac{\Gamma_{e} A_{e}}{\delta x_{P E}}$ & $\frac{\Gamma_{s} A_{s}}{\delta x_{S P}}$ & $\frac{\Gamma_{n} A_{n}}{\delta x_{P N}}$ & $a_{W}+a_{E}+a_{S}+a_{N}-S_{p}$ \\
\hline
\end{tabular}

The variable $\mathrm{A}$ is the cross sectional area, and $\mathrm{S}$ components are source terms. For two dimensions, the face areas in a two-dimensional case are $A_{w}=A_{e}=\triangle y ; A_{n}=$ $A_{s}=\triangle x$. The distribution of the property $\phi$ is given by obtaining a discretized equation at each grid node of the two dimensional domain. [25]

Step 3: Solution of equations: The discretized equations must be found for each nodal point. Control volumes beside the domain boundaries are modified to incorporate the boundary conditions. The system of equations is solved using a matrix 
solution technique to obtain the distribution of the property $\phi$ at the nodal points. [25]

\section{D.2.1 Central Differencing Finite Volume Advection Scheme}

This scheme is an approximation used for diffusion terms appearing on the right side of the equation and trying linear interpolation to compute cell face values for convective terms on the left side of the equation. [25] The central differencing expression for the discretized convection-diffusion equation are:

$$
a_{P} \phi_{P}=a_{W} \phi_{P}=a_{W} \phi_{W}+a_{E} \phi_{E}
$$

where

\begin{tabular}{|l|l|l|}
\hline$a_{W}$ & $a_{E}$ & $a_{P}$ \\
\hline$D_{w}+\frac{F_{w}}{2}$ & $D_{e}+\frac{F_{e}}{2}$ & $a_{W}+a_{E}+\left(F_{e}-F_{w}\right)$ \\
\hline
\end{tabular}

This equation can be discretized for all grid nodes, yielding a set of algebraic equations that can be solved to obtain distribution of the transported property $\phi$. [25] Discretization scheme properties are fundamental properties that determine whether numerical results will be physically realistic. The most important ones are conservativeness, boundedness, and transportiveness. Discretized conservative equations involves fluxes of $\phi$ through control volume faces.

The major inadequacy of the central differencing scheme is its ability to identi $F_{y}$ flow direction. Values of $\phi$ at a west cell face are equally influenced by both $\phi_{P}$ and $\phi_{W}$, when a strongly convective flow from west to east should receive a much stronger influence from node $\mathrm{W}$ than node $\mathrm{P}$. [25] 


\section{D.3 LES Flow Around Bluff Bodies}

The standard turbulence models used in most codes are inadequate, specifically for flow around bluff bodies such as buildings. Unsteady techniques, like large eddy simulation (LES), are more appropriate and sometimes required for correct identification of critical unsteady features of flows. Significant loads created by extreme low pressure regions within the flow by the shedding of concentrated vorticity can be identified using LES since it directly computes the dynamically significant flow structures. LES has several retaining issues that must be specifically resolved. Such issues are rough wall boundary conditions and the definitions of inflow conditions. LES can capture major features of the flow around bluff bodies submerged in a thick, rough walled, simulated atmospheric boundary layer. Separate precursor simulations of the approaching flow must be performed. [26]

The governing equations for LES consider an incompressible fluid of kinematic viscosity $v$ in motion with kinematic pressure $\mathrm{p}$ and velocity $u_{i}=(u, v, w)$, along the Cartesian axes $x_{i}=(x, y, z)$. To resolve the filtered velocity field, scale-limited solutions are required of the Navier-Stokes equations. LES of bluff body flows require generation of inflow turbulence, analogous to the upwind environment of a building. The amount of wind shear, level of turbulence intensity, and integral scale of the turbulence must be reproduced. A precursor simulation is ideal because the inflow it generates should contain the natural physical coherent structures non-artificially. The fluctuating velocity field $(\mathrm{u}, \mathrm{v}, \mathrm{w})$ at the location of the precursor's outflow boundary is sampled at each time step and saved in a database. The turbulent inflow for the bluff body simulation uses this data. [26] This thesis will utilize this method by running a steady state simulation and using its results as the initial values for the final large eddy simulation. 